\title{
Color-allowed bottom baryon to charmed baryon nonleptonic decays
}

\author{
Chun-Khiang Chua \\ Department of Physics and Center for High Energy Physics, Chung Yuan Christian University, \\ Chung-Li, Taiwan 320, Republic of China
}

(Received 3 December 2018; published 18 January 2019)

\begin{abstract}
We study color allowed $\Lambda_{b} \rightarrow \Lambda_{c}^{(*, * *)} M^{-}, \Xi_{b} \rightarrow \Xi_{c}^{(* *)} M^{-}$, and $\Omega_{b} \rightarrow \Omega_{c}^{(*)} M^{-}$decays with $M=\pi$, $K, \rho, K^{*}, D, D_{s}, D^{*}, D_{s}^{*}, a_{1}, \quad \Lambda_{c}^{(*, *)}=\Lambda_{c}, \Lambda_{c}(2595), \Lambda_{c}(2765), \Lambda_{c}(2940), \quad \Xi_{c}^{(* *)}=\Xi_{c}, \Xi_{c}(2790)$, and $\Omega_{c}^{(*)}=\Omega_{c}, \Omega_{c}(3090)$, in this work. There are three types of transitions, namely $\mathcal{B}_{b}\left(\overline{\mathbf{3}}_{\mathbf{f}}, 1 / 2^{+}\right)$to $\mathcal{B}_{c}\left(\overline{\mathbf{3}}_{\mathbf{f}}, 1 / 2^{+}\right), \mathcal{B}_{b}\left(\mathbf{6}_{\mathbf{f}}, 1 / 2^{+}\right)$to $\mathcal{B}_{c}\left(\mathbf{6}_{\mathbf{f}}, 1 / 2^{+}\right)$, and $\mathcal{B}_{b}\left(\overline{\mathbf{3}}_{\mathbf{f}}, 1 / 2^{+}\right)$to $\mathcal{B}_{c}\left(\overline{\mathbf{3}}_{\mathbf{f}}, 1 / 2^{-}\right)$transitions. The bottom baryon to charmed baryon form factors are calculated using the light-front quark model. Decay rates and up-down asymmetries are predicted using naïve factorization and can be checked experimentally. We find that in $\mathcal{B}_{b} \rightarrow \mathcal{B}_{c} P$ decays, rates in $\mathcal{B}_{b}\left(\mathbf{6}_{\mathbf{f}}, 1 / 2^{+}\right) \rightarrow \mathcal{B}_{c}\left(\mathbf{6}_{\mathbf{f}}, 1 / 2^{+}\right)$[type (ii)] transition are smaller than those in $\mathcal{B}_{b}\left(\overline{\mathbf{3}}_{\mathbf{f}}, 1 / 2^{+}\right) \rightarrow \mathcal{B}_{c}\left(\overline{\mathbf{3}}_{\mathbf{f}}, 1 / 2^{+}\right)$[type (i)] transition, but similar to those in $\mathcal{B}_{b}\left(\overline{\mathbf{3}}_{\mathbf{f}}, 1 / 2^{+}\right) \rightarrow \mathcal{B}_{c}\left(\overline{\mathbf{3}}_{\mathbf{f}}, 1 / 2^{-}\right)$ [type (iii)] transition, while in $\mathcal{B}_{b} \rightarrow \mathcal{B}_{c} V, \mathcal{B}_{c} A$ decays, rates in $\mathcal{B}_{b}\left(\mathbf{6}_{\mathbf{f}}, 1 / 2^{+}\right) \rightarrow \mathcal{B}_{c}\left(\mathbf{6}_{\mathbf{f}}, 1 / 2^{+}\right.$) [type (ii)] transition are much smaller than those in $\mathcal{B}_{b}\left(\overline{\mathbf{3}}_{\mathbf{f}}, 1 / 2^{+}\right) \rightarrow \mathcal{B}_{c}\left(\overline{\mathbf{3}}_{\mathbf{f}}, 1 / 2^{+}\right)$[type (i)] transition and are also smaller than those in $\mathcal{B}_{b}\left(\overline{\mathbf{3}}_{\mathbf{f}}, 1 / 2^{+}\right) \rightarrow \mathcal{B}_{c}\left(\overline{\mathbf{3}}_{\mathbf{f}}, 1 / 2^{-}\right)$[type (iii)] transition. For the up-down asymmetries, the signs are mostly negative, except for those in the $\mathcal{B}_{b}\left(\mathbf{6}_{\mathbf{f}}, 1 / 2^{+}\right) \rightarrow \mathcal{B}_{c}\left(\mathbf{6}_{\mathbf{f}}, 1 / 2^{+}\right)$[type (ii)] transition. Most of these asymmetries are large in sizes. The study on these decay modes may shed light on the quantum numbers of some of the charmed baryons as the decays depend on the bottom baryon to charmed baryon form factors, which are sensitive to the configurations of the final state charmed baryons.
\end{abstract}

DOI: $10.1103 /$ PhysRevD.99.014023

\section{INTRODUCTION}

As noted in the review of Particle Data Group (PDG) (see the review by C. G. Wohl in [1]), there are 24 singly charmed baryons and nine singly bottom baryons. ${ }^{1}$ Among them $\Lambda_{c}(2864)$ and five $\Omega_{c}$ states, namely $\Omega_{c}(3000)^{0}$, $\Omega_{c}(3050)^{0}, \quad \Omega_{c}(3066)^{0}, \quad \Omega_{c}(3090)^{0}$, and $\Omega_{c}(3119)^{0}$, are newly discovered by $\mathrm{LHCb}$ in year $2017[2,3]$. The quantum numbers of 9 out of the 24 charmed baryons are unspecified. These include the above five $\Omega_{c}$ states, $\Sigma_{c}(2800)^{++,+, 0}, \Xi_{c}(3055)^{+, 0}, \Xi_{c}(3080)^{+, 0}$, and $\Xi_{c}(2970)^{+, 0}$ baryons. Note that, in addition to the above states, some other states, including $\Lambda_{c}(2765)^{+}$[or $\left.\Sigma_{c}(2765)\right], \Xi_{c}(2930)^{0}$, and $\Xi_{c}(3123)^{+}$, are not included in the short review and their quantum numbers remain unspecified as well. Furthermore PDG stated that $\frac{3-}{2}$ is the favored quantum number of $\Lambda_{c}(2940)^{+}$, but it is not certain [1], while the authors of Ref. [4] argued that it should be a

\footnotetext{
${ }^{1}$ In the review by Wohl, particles in the same isospin-multiplet, such as $\Sigma_{c}^{++,+, 0}$, are not counted separately.

Published by the American Physical Society under the terms of the Creative Commons Attribution 4.0 International license. Further distribution of this work must maintain attribution to the author(s) and the published article's title, journal citation, and DOI. Funded by SCOAP ${ }^{3}$.
}

$\frac{1}{2}-$ state. It is not surprising that there are various suggestions on the quantum numbers of the newly discovered $\Omega_{c}$ states, see e.g., [4-11]. It is, therefore, of great important to identify the quantum numbers of these states and understand their properties.

Among low lying singly bottom baryons, only $\Lambda_{b}, \Xi_{b}$ and $\Omega_{b}$ decay weakly [1]. Several color allowed $\Lambda_{b} \rightarrow \Lambda_{c} P$ decay rates with $P=\pi, K, D, D_{s}$ were reported by $\mathrm{LHCb}$ in year 2014 [12-14]. We expect more to come in the near future. It will be interesting and timely to study weak decays of singly bottom baryons to final states involving singly charmed baryons. In general, baryon decays are complicate processes. Nevertheless, when the transition only involve the heavy quarks, namely $b \rightarrow c$ transition, while the light quarks are spectating, the decay processes are easier. Accordingly we will study color allowed $\Lambda_{b}^{0} \rightarrow \Lambda_{c}^{(* * *)} M, \Xi_{b} \rightarrow \Xi_{c}^{(* *)} M$, and $\Omega_{b} \rightarrow \Omega_{c}^{(*)} M$ decays with $M=\pi, K, \rho, K^{*}, D, D_{s}, D^{*}, D_{s}^{*}, a_{1}, \Lambda_{c}^{(*, * *)}=$ $\Lambda_{c}, \Lambda_{c}(2595), \Lambda_{c}(2765), \Lambda_{c}(2940), \Xi_{c}^{(*, *)}=\Xi_{c}, \Xi_{c}(2790)$, and $\Omega_{c}^{(*)}=\Omega_{c}, \Omega_{c}$ (3090). In this work, we follow Ref. [4] to take $\Lambda_{c}(2765), \Lambda_{c}(2940)$, and $\Omega_{c}(3090)$ as a radial excited $s$-wave $1 / 2^{+}$state, a radial excited $p$-wave $1 / 2^{-}$ state, and a radial excite $s$-wave $1 / 2^{+}$state, respectively. There are other quantum number assignments. For example, as noted in the previous paragraph, PDG and LHCb 
prefer $3 / 2^{-}$for quantum number of $\Lambda_{c}(2940)[1,2]$ and several authors consider $\Omega_{c}(3090)$ as a candidate of a $p$ wave state, usually with a spin higher than $1 / 2$ [5-10]. It should be noted that some authors also consider $\Omega_{c}(3090)$ as a $1 / 2^{+}$state $[6,11]$. The study on these $\mathcal{B}_{b} \rightarrow \mathcal{B}_{c} M$ decays may shed light on the quantum numbers of $\Lambda_{c}(2765), \Lambda_{c}(2940)$, and $\Omega_{c}(3090)$, as the decays depend on the bottom baryon to charmed baryon form factors, which are sensitive to the configurations of the final state charmed baryons. We will use the light-front quark model to calculate the form factors. The formalism is similar to the one in Ref. [15], which was used to study a different problem. For some other studies on some of the above modes or on some related form factors in various approaches, one is referred to [16-30].

We begin with a brief review of the spectroscopy of charmed and bottom baryon states and discuss their possible spin-parity quantum numbers and inner structure in Sec. II. In Sec. III we work out the formulas for form factors in the light-front quark model. We present our numerical results for form factors, decay rates and up-down asymmetries in Sec. IV. Section V comes to our conclusions. Appendix A is prepared to give some details of the derivations of the vertex functions, while some discussions on the technical issue of obtaining form factors are collected in Appendix B.

\section{SPECTROSCOPY OF SINGLY CHARMED AND BOTTOM BARYONS}

In this section we briefly review the spectroscopy of singly charmed and bottom baryons. Our discussion follows closely to those in $[31,32]$. The singly charmed or bottom baryon is composed of a charmed quark or a bottom quark and two light quarks. We will discuss the allowed quantum numbers for the light quark system before the brief review.

\section{A. Allowed quantum numbers for the light quark system}

From Fermi statistics the wave function of the light quarks needs to be antisymmetry under permutation. As the charm or bottom quark is a color triplet $\mathbf{3}_{\mathbf{c}}$, the diquark system, consists of the two light quarks, can only be an anticolor triplet $\overline{\mathbf{3}}_{\mathbf{c}}$ state, which is antisymmetric [denoted as $\left.\left(\overline{\mathbf{3}}_{\mathbf{c}}\right)_{A}\right]$ under permutation of the two light quarks. The remaining part of the diquark wave function consists of

$$
(\psi(\text { space }) \times \psi(\text { flavor }) \times \psi(\text { spin }))_{S},
$$

must be symmetry under permutation.

The spin of the light quarks can be in a symmetric triplet state $\left(\mathbf{3}_{\mathbf{s p}}\right)_{S}\left(S_{l}=1\right)$ or an antisymmetric singlet state $\left(\mathbf{1}_{\mathbf{s p}}\right)_{A}\left(S_{l}=0\right)$. Under permutation, the spin wave function picks up an phase factor

$$
\psi(\text { spin }) \rightarrow(-)^{S_{l}+1} \psi(\text { spin }) .
$$

Given that each light quark is a triplet of the flavor $\mathrm{SU}(3)$ and $\mathbf{3}_{\mathbf{f}} \times \mathbf{3}_{\mathbf{f}}=\left(\overline{\mathbf{3}}_{\mathbf{f}}\right)_{A}+\left(\mathbf{6}_{\mathbf{f}}\right)_{S}$, there are two different SU(3) multiplets of charmed or bottom baryons: a symmetric sextet $\left(\mathbf{6}_{\mathbf{f}}\right)_{S}$ and an antisymmetric antitriplet $\left(\overline{\mathbf{3}}_{\mathbf{f}}\right)_{A}$. The isosinglet $\Lambda_{Q}$ and isodoublet $\Xi_{Q}$ form a $\left(\overline{\mathbf{3}}_{\mathbf{f}}\right)_{A}$ representation, while the $\Omega_{Q}$, isodoublet $\Xi_{Q}^{\prime}$, and isotriplet $\Sigma_{Q}$ form a $\left(\mathbf{6}_{f}\right)_{S}$ representation. ${ }^{2}$ Under permutation, the flavor wave function picks up an phase factor

$$
\psi(\text { flavor }) \rightarrow(-)^{N_{f}} \psi(\text { flavor }),
$$

with $N_{f}=3,6$ for $\overline{\mathbf{3}}_{\mathbf{f}}, \mathbf{6}_{\mathbf{f}}$, respectively.

In the quark model, the orbital angular momentum of the light diquark can be decomposed into $\mathbf{L}_{\ell}=\mathbf{L}_{k}+\mathbf{L}_{K}$, where $\mathbf{L}_{k}$ is the orbital angular momentum between the two light quarks and $\mathbf{L}_{K}$ the orbital angular momentum between the diquark (the light quark pair) and the heavy quark. Roughly speaking, we have

$$
\psi(\text { space }) \sim R_{n}(|\vec{K}|) \times Y_{L_{k} m_{k}}(\vec{k}) Y_{L_{K} m_{K}}(\vec{K}),
$$

where $R_{n}$ is the radial wave function, $Y_{l m}$ is the spherical harmonics, $\vec{k}$ is basically the relative momentum of the two light quarks and $\vec{K}$ is the relative momentum of the heavy quark and the diquark system. ${ }^{3}$ In the above equation, we do not show explicitly the Clebsch-Gordan coefficient and the Wigner rotation (see later discussion), as the rest frame of the whole system and the rest frame of the diquark system are not identical. Nevertheless the above wave function can still be used as an book keeping devise for working out the allowed quantum numbers.

The angular momentum of the diquark system, without taking into account the orbital momentum of the $Q-[q q]$ system, is

$$
\vec{S}_{[q q]}=\vec{L}_{k}+\vec{S}_{q q},
$$

with $S_{[q q]}$ given by $\left|L_{k}-S_{q q}\right|, \ldots, L_{k}+S_{q q}$. Note that $\vec{S}_{q q}$ is the spin of the light quark pair without taking into account the orbital momentum between them. The combination of $\vec{S}_{[q q]}$ is better when viewing the diquark as a

\footnotetext{
${ }^{2}$ We have followed the Particle Data Group's convention [1] to use a prime to distinguish the isodoublet in the $\mathbf{6}_{\mathbf{f}}$ from the one in the $\overline{\mathbf{3}}_{\mathbf{f}}$.

${ }^{3}$ Explicitly, we have $k^{\mu} \equiv\left(p_{1}-p_{2}\right)^{\mu}-\left(p_{1}+p_{2}\right)^{\mu}\left[\left(p_{1}+\right.\right.$ $\left.\left.p_{2}\right) \cdot\left(p_{1}-p_{2}\right) /\left(p_{1}+p_{2}\right)^{2}\right]$ and $K^{\mu} \equiv\left(p_{1}+p_{2}-p_{3}\right)^{\mu}-P^{\mu}[P$. $\left.\left(p_{1}+p_{2}-p_{3}\right) / P^{2}\right]$ with $p_{1}, p_{2}$ and $p_{3}$ the momenta of the light quarks and the heavy quark, respectively, and $P \equiv p_{1}+p_{2}+p_{3}$. Note the above constructions in $K$ and $k$ are to make sure that in the rest frame of the light-quark system and in the whole baryon system, we have $k=(0, \vec{k})$ and $K=(0, \vec{K})$, respectively.
} 
TABLE I. Allowed quantum number of the diquark system satisfying Fermi statistics. $\vec{S}_{[q q]}=\vec{L}_{k}+\vec{S}_{q q}$ is the angular momentum of the diquark system, without taking into account the orbital momentum of the $Q-[q q]$ system $\left(\vec{L}_{K}\right)$. The parity eigenvalues of the diquark $[q q]$ system and the $Q q q$ system are given by $(-)^{L_{k}}$ and $(-)^{L_{k}+L_{K}}$, respectively.

\begin{tabular}{lccccccc}
\hline \hline$\psi($ color $)$ & $L_{K}$ & $L_{k}$ & $\psi($ flavor $)$ & $\psi($ spin $)$ & $S_{[q q]}$ & Parity $([q q])$ & Parity $(Q q q)$ \\
\hline$\left(\overline{\mathbf{3}}_{\mathbf{c}}\right)_{A}$ & even & even & $\left(\overline{\mathbf{3}}_{\mathbf{f}}\right)_{A}$ & $\left(\mathbf{1}_{\text {sp }}\right)_{A}$ & $L_{k}$ & + & + \\
$\left(\overline{\mathbf{3}}_{\mathbf{c}}\right)_{A}$ & even & even & $\left(\mathbf{6}_{\mathbf{f}}\right)_{S}$ & $\left(\mathbf{3}_{\text {sp }}\right)_{S}$ & $\left|L_{k}-1\right|, \ldots, L_{k}+1$ & + & + \\
$\left(\overline{\mathbf{3}}_{\mathbf{c}}\right)_{A}$ & odd & odd & $\left(\overline{\mathbf{3}}_{\mathbf{f}}\right)_{A}$ & $\left(\mathbf{3}_{\text {sp }}\right)_{S}$ & $L_{k}-1, L_{k}, L_{k}+1$ & - & + \\
$\left(\overline{\mathbf{3}}_{\mathbf{c}}\right)_{A}$ & odd & odd & $\left(\mathbf{6}_{\mathbf{f}}\right)_{S}$ & $\left(\mathbf{1}_{\text {sp }}\right)_{A}$ & $L_{k}$ & - & + \\
$\left(\overline{\mathbf{3}}_{\mathbf{c}}\right)_{A}$ & even & odd & $\left(\overline{\mathbf{3}}_{\mathbf{f}}\right)_{A}$ & $\left(\mathbf{3}_{\text {sp }}\right)_{S}$ & $L_{k}-1, L_{k}, L_{k}+1$ & - & - \\
$\left(\overline{\mathbf{3}}_{\mathbf{c}}\right)_{A}$ & even & odd & $\left(\mathbf{6}_{\mathbf{f}}\right)_{S}$ & $\left(\mathbf{1}_{\text {sp }}\right)_{A}$ & $L_{k}$ & - & - \\
$\left(\overline{\mathbf{3}}_{\mathbf{c}}\right)_{A}$ & odd & even & $\left(\overline{\mathbf{3}}_{\mathbf{f}}\right)_{A}$ & $\left(\mathbf{1}_{\mathbf{s p}}\right)_{A}$ & $L_{k}$ & + & - \\
$\left(\overline{\mathbf{3}}_{\mathbf{c}}\right)_{A}$ & odd & even & $\left(\mathbf{6}_{\mathbf{f}}\right)_{S}$ & $\left(\mathbf{3}_{\mathbf{s p}}\right)_{S}$ & $\left|L_{k}-1\right|, \ldots, L_{k}+1$ & + & - \\
\hline \hline
\end{tabular}

sub-system, i.e., one may have scalar diquark, axial-vector diquark and so on. The angular momentum of the diquark system, with the orbital momentum of the $Q-[q q]$ system, is

$$
\vec{J}_{l}=\vec{S}_{[q q]}+\vec{L}_{K}
$$

with $J_{l}$ given by $\left|S_{[q q]}-L_{k}\right|, \ldots, S_{[q q]}+L_{k}$. Consequently, the total angular momentum is

$$
\begin{aligned}
\vec{J}_{Q q q} & =\vec{S}_{Q}+\vec{L}_{k}+\vec{S}_{q q}+\vec{L}_{K} \\
& =\vec{S}_{Q}+\vec{S}_{[q q]}+\vec{L}_{K}=\vec{S}_{Q}+\vec{J}_{l} .
\end{aligned}
$$

Under permutation of the light quark momenta, $p_{1} \leftrightarrow p_{2}$, we have $\vec{k} \rightarrow-\vec{k}$ and $\vec{K} \rightarrow \vec{K}$, while under party we have $\vec{k} \rightarrow-\vec{k}$ and $\vec{K} \rightarrow-\vec{K}$. Consequently, using the well known symmetry property of $Y_{l m}$, under the permutation, the space part wave function, see Eq. (4), transforms as

$$
\psi(\text { space }) \rightarrow(-)^{L_{k}} \psi(\text { space }),
$$

while under parity, it transforms as

$$
\psi(\text { space }) \rightarrow(-)^{L_{k}+L_{K}} \psi(\text { space }) .
$$

The parity eigenvalues of the $[q q]$ diquark and the whole $Q q q$ systems are given by $(-)^{L_{k}}$ and $(-)^{L_{k}+L_{K}}$, respectively.

Putting all of these together, under permutation of the light quarks, we have

$$
\begin{aligned}
& \psi(\text { color }) \times \psi(\text { space }) \times \psi(\text { flavor }) \times \psi(\text { spin }) \\
& \rightarrow-(-)^{L_{k}}(-)^{N_{f}}(-)^{S_{l}+1} \psi(\text { color }) \\
& \quad \times \psi(\text { space }) \times \psi(\text { flavor }) \times \psi(\text { spin }) .
\end{aligned}
$$

Fermi statistics requires the wave function to be antisymmetric giving the following constraint:

$$
(-)^{L_{k}+N_{f}+S_{l}}=-1 \text {. }
$$

The quantum numbers of all possible allowed configurations of the diquark system satisfying the Fermi statistic are shown in Table I. The corresponding parity eigenvalues of the diquark and the heavy baryons are also shown.

\section{B. Charmed baryons}

The observed mass spectra and decay widths of charmed baryons are summarized in Table II. The $J^{P}$ quantum numbers of $\Lambda_{c}^{+}, \Lambda_{c}(2595)^{+}, \Lambda_{c}(2860)^{+}, \Lambda_{c}(2880)^{+}$, $\Lambda_{c}(2940)^{+}$, and $\Sigma_{c}(2455)$, are determined up to different levels of certainty, while the $J^{P}$ quantum numbers given in Table II other states are either from quark model predictions or totally undetermined. In fact, there are 16 states out of 40 states in Table II having unknown quantum numbers.

In Table III configurations with $L_{k}+L_{K}=0,1,2$ for charmed baryons are shown. The quantum number assignments are from Tables I and II, while those with $(\dagger)$ are taken from Ref. [4]. Only several multiplets are well established. These include the $J^{P}=\frac{1}{2}^{+} \overline{\mathbf{3}}_{\mathrm{f}}$ states: $\left(\Lambda_{c}^{+}, \Xi_{c}^{+}, \Xi_{c}^{0}\right), J^{P}=\frac{1}{2}-$ $\overline{\mathbf{3}}_{\mathbf{f}}$ states: $\left(\Lambda_{c}(2595)^{+}, \Xi_{c}(2790)^{+}, \Xi_{c}(2790)^{0}\right) ; J^{P}=\frac{3}{2} \overline{\mathbf{3}}_{\mathbf{f}}$ states: $\left(\Lambda_{c}(2625)^{+}, \Xi_{c}(2815)^{+}, \Xi_{c}(2815)^{0}\right) ; J^{P}=\frac{1}{2}^{+}$and $\frac{3}{2}^{+} \boldsymbol{6}_{\mathbf{f}}$ states: $\left(\Omega_{c}, \Sigma_{c}, \Xi_{c}^{\prime}\right)$ and $\left(\Omega_{c}^{*}, \Sigma_{c}^{*}, \Xi_{c}^{\prime *}\right)$, respectively. Reference [4] makes further suggestions on the classification on some other states. As noted previously PDG and LHCb assign $\Lambda_{c}(2940)^{+}$as a $\frac{3}{2}-$ state $[1,2]$, while the authors of Ref. [4] take it as a $\frac{1}{2}-$ state. We follow the suggestions of Ref. [4] on the quantum numbers of $\Lambda_{c}(2940)^{+}$and some other states. Note that other quantum number assignments on the newly observed $\Omega_{c}^{(*, *)}$ states, such as those advocated in Refs. [5-10], are not shown in the table.

From Table III we see that there are plenty of states in the $L_{k}+L_{K}=0,1,2$ sector to be discovered.

\section{Bottom baryons}

The observed mass spectra and decay widths of bottom baryons are summarized in Table IV. Note that except $\Xi_{b}^{\prime}(5935)^{-}$and $\Xi_{b}(5955)^{-}$other $J^{P}$ quantum numbers 
TABLE II. Mass spectra and widths (in units of MeV unless specified) of charmed baryons. Experimental values and $J^{P}$ are taken from the Particle Data Group [1]. The quantum number of $\Lambda_{c}(2940)$ can be different from the one shown in the table, see text for more details.

\begin{tabular}{|c|c|c|c|c|c|c|c|c|}
\hline State & $J^{P}$ & $n$ & $\left(L_{K}, L_{k}\right)$ & $S_{[q q]}^{P}$ & $J_{\ell}^{P_{\ell}}$ & Mass & Width & Decay modes \\
\hline$\Lambda_{c}^{+}$ & $\frac{1}{2}^{+}$ & 1 & $(0,0)$ & $0^{+}$ & $0^{+}$ & $2286.46 \pm 0.14$ & & weak \\
\hline$\Lambda_{c}(2595)^{+}$ & $\frac{1}{2}-$ & 1 & $(1,0)$ & $0^{+}$ & $1^{-}$ & $2592.25 \pm 0.28$ & $2.6 \pm 0.6$ & $\Lambda_{c} \pi \pi, \Sigma_{c} \pi$ \\
\hline$\Lambda_{c}(2625)^{+}$ & $\frac{3}{2}-$ & 1 & $(1,0)$ & $0^{+}$ & $1^{-}$ & $2628.11 \pm 0.19$ & $<0.97$ & $\Lambda_{c} \pi \pi, \Sigma_{c} \pi$ \\
\hline$\Lambda_{c}(2765)^{+}$ & $?$ & $?$ & $?$ & $?$ & $?$ & $2766.6 \pm 2.4$ & 50 & $\Sigma_{c} \pi, \Lambda_{c} \pi \pi$ \\
\hline$\Lambda_{c}(2860)^{+}$ & $\frac{3}{2}^{+}$ & 1 & $(2,0)$ & $0^{+}$ & $2^{+}$ & $2856.1_{-6.0}^{+2.3}$ & $68_{-22}^{+12}$ & $\Sigma_{c}^{(*)} \pi, D^{0} p, D^{+} n$ \\
\hline$\Lambda_{c}(2880)^{+}$ & $\frac{5}{2}+$ & 1 & $(2,0)$ & $0^{+}$ & $2^{+}$ & $2881.63 \pm 0.24$ & $5.6_{-0.6}^{+0.8}$ & $\Sigma_{c}^{(*)} \pi, \Lambda_{c} \pi \pi, D^{0} p$ \\
\hline$\Lambda_{c}(2940)^{+}$ & $\frac{3}{2}-$ & 2 & $(1,0)$ & $0^{+}$ & $1^{-}$ & $2939.6_{-1.5}^{+1.3}$ & $20_{-5}^{+6}$ & $\Sigma_{c}^{(*)} \pi, \Lambda_{c} \pi \pi, D^{0} p$ \\
\hline$\Sigma_{c}(2455)^{++}$ & $\frac{1}{2}+$ & 1 & $(0,0)$ & $1^{+}$ & $1^{+}$ & $2453.97 \pm 0.14$ & $1.89_{-0.18}^{+0.09}$ & $\Lambda_{c} \pi$ \\
\hline$\Sigma_{c}(2455)^{+}$ & $\frac{1}{2}+$ & 1 & $(0,0)$ & $1^{+}$ & $1^{+}$ & $2452.9 \pm 0.4$ & $<4.6$ & $\Lambda_{c} \pi$ \\
\hline$\Sigma_{c}(2455)^{0}$ & $\frac{1}{2}^{+}$ & 1 & $(0,0)$ & $1^{+}$ & $1^{+}$ & $2453.75 \pm 0.14$ & $1.83_{-0.19}^{+0.11}$ & $\Lambda_{c} \pi$ \\
\hline$\Sigma_{c}(2520)^{++}$ & $\frac{3}{2}+$ & 1 & $(0,0)$ & $1^{+}$ & $1^{+}$ & $2518.41_{-0.19}^{+0.21}$ & $14.78_{-0.40}^{+0.30}$ & $\Lambda_{c} \pi$ \\
\hline$\Sigma_{c}(2520)^{+}$ & $\frac{3}{2}+$ & 1 & $(0,0)$ & $1^{+}$ & $1^{+}$ & $2517.5 \pm 2.3$ & $<17$ & $\Lambda_{c} \pi$ \\
\hline$\Sigma_{c}(2520)^{0}$ & $\frac{3}{2}+$ & 1 & $(0,0)$ & $1^{+}$ & $1^{+}$ & $2518.48 \pm 0.20$ & $15.3_{-0.5}^{+0.4}$ & $\Lambda_{c} \pi$ \\
\hline$\Sigma_{c}(2800)^{++}$ & $?$ & $?$ & $?$ & $?$ & $?$ & $2801_{-6}^{+4}$ & $75_{-17}^{+22}$ & $\Lambda_{c} \pi, \Sigma_{c}^{(*)} \pi, \Lambda_{c} \pi \pi$ \\
\hline$\Sigma_{c}(2800)^{+}$ & $?$ & $?$ & $?$ & $?$ & $?$ & $2792_{-5}^{+14}$ & $62_{-40}^{+60}$ & $\Lambda_{c} \pi, \Sigma_{c}^{(*)} \pi, \Lambda_{c} \pi \pi$ \\
\hline$\Sigma_{c}(2800)^{0}$ & $?$ & $?$ & $?$ & $?$ & $?$ & $2806_{-7}^{+5}$ & $72_{-15}^{+22}$ & $\Lambda_{c} \pi, \Sigma_{c}^{(*)} \pi, \Lambda_{c} \pi \pi$ \\
\hline$\Xi_{c}^{+}$ & $\frac{1}{2}+$ & 1 & $(0,0)$ & $0^{+}$ & $0^{+}$ & $2467.87 \pm 0.30$ & & weak \\
\hline$\Xi_{c}^{0}$ & $\frac{1}{2}+$ & 1 & $(0,0)$ & $0^{+}$ & $0^{+}$ & $2470.87_{-0.31}^{+0.28}$ & & weak \\
\hline$\Xi_{c}^{\prime+}$ & $\frac{1}{2}^{+}$ & 1 & $(0,0)$ & $1^{+}$ & $1^{+}$ & $2577.4 \pm 1.2$ & & $\Xi_{c} \gamma$ \\
\hline$\Xi_{c}^{\prime 0}$ & $\frac{1}{2}+$ & 1 & $(0,0)$ & $1^{+}$ & $1^{+}$ & $2578.8 \pm 0.5$ & & $\Xi_{c} \gamma$ \\
\hline$\Xi_{c}(2645)^{+}$ & $\frac{3}{2}+$ & 1 & $(0,0)$ & $1^{+}$ & $1^{+}$ & $2645.53 \pm 0.31$ & $2.14 \pm 0.19$ & $\Xi_{c} \pi$ \\
\hline$\Xi_{c}(2645)^{0}$ & $\frac{3}{2}+$ & 1 & $(0,0)$ & $1^{+}$ & $1^{+}$ & $2646.32 \pm 0.31$ & $2.35 \pm 0.22$ & $\Xi_{c} \pi$ \\
\hline$\Xi_{c}(2790)^{+}$ & $\frac{1}{2}-$ & 1 & $(1,0)$ & $0^{+}$ & $1^{-}$ & $2792.0 \pm 0.5$ & $8.9 \pm 1.0$ & $\Xi_{c}^{\prime} \pi$ \\
\hline$\Xi_{c}(2790)^{0}$ & $\frac{1}{2}-$ & 1 & $(1,0)$ & $0^{+}$ & $1^{-}$ & $2792.8 \pm 1.2$ & $10.0 \pm 1.1$ & $\Xi_{c}^{\prime} \pi$ \\
\hline$\Xi_{c}(2815)^{+}$ & $\frac{3}{2}-$ & 1 & $(1,0)$ & $0^{+}$ & $1^{-}$ & $2816.67 \pm 0.31$ & $2.43 \pm 0.26$ & $\Xi_{c}^{*} \pi, \Xi_{c} \pi \pi, \Xi_{c}^{\prime} \pi$ \\
\hline$\Xi_{c}(2815)^{0}$ & $\frac{3}{2}-$ & 1 & $(1,0)$ & $0^{+}$ & $1^{-}$ & $2820.22 \pm 0.32$ & $2.54 \pm 0.25$ & $\Xi_{c}^{*} \pi, \Xi_{c} \pi \pi, \Xi_{c}^{\prime} \pi$ \\
\hline$\Xi_{c}(2930)^{0}$ & $?$ & $?$ & $?$ & $?$ & $?$ & $2931 \pm 6$ & $36 \pm 13$ & $\Lambda_{c} \bar{K}$ \\
\hline$\Xi_{c}(2970)^{+}$ & $?$ & $?$ & $?$ & $?$ & $?$ & $2969.4 \pm 0.8$ & $20.9_{-3.5}^{+2.4}$ & $\Sigma_{c} \bar{K}, \Lambda_{c} \bar{K} \pi, \Xi_{c} \pi \pi$ \\
\hline$\Xi_{c}(2970)^{0}$ & $?$ & $?$ & $?$ & $?$ & $?$ & $2967.8 \pm 0.8$ & $28.1_{-4.0}^{+3.4}$ & $\Sigma_{c} \bar{K}, \Lambda_{c} \bar{K} \pi, \Xi_{c} \pi \pi$ \\
\hline$\Xi_{c}(3055)^{+}$ & $?$ & $?$ & $?$ & $?$ & $?$ & $3055.9 \pm 0.4$ & $7.8 \pm 1.9$ & $\Sigma_{c} \bar{K}, \Lambda_{c} \bar{K} \pi, D \Lambda$ \\
\hline$\Xi_{c}(3080)^{+}$ & $?$ & $?$ & $?$ & $?$ & $?$ & $3077.2 \pm 0.4$ & $3.6 \pm 1.1$ & $\Sigma_{c} \bar{K}, \Lambda_{c} \bar{K} \pi, D \Lambda$ \\
\hline$\Xi_{c}(3080)^{0}$ & $?$ & $?$ & $?$ & $?$ & $?$ & $3079.9 \pm 1.4$ & $5.6 \pm 2.2$ & $\Sigma_{c} \bar{K}, \Lambda_{c} \bar{K} \pi, D \Lambda$ \\
\hline$\Xi_{c}(3123)^{+}$ & $?$ & $?$ & $?$ & $?$ & $?$ & $3122.9 \pm 1.3$ & $4 \pm 4$ & $\Sigma_{c}^{*} \bar{K}, \Lambda_{c} \bar{K} \pi$ \\
\hline$\Omega_{c}^{0}$ & $\frac{1}{2}^{+}$ & 1 & $(0,0)$ & $1^{+}$ & $1^{+}$ & $2695.2 \pm 1.7$ & & weak \\
\hline$\Omega_{c}(2770)^{0}$ & $\frac{3}{2}+$ & 1 & $(0,0)$ & $1^{+}$ & $1^{+}$ & $2765.9 \pm 2.0$ & & $\Omega_{c} \gamma$ \\
\hline$\Omega_{c}(3000)^{0}$ & $?$ & $?$ & $?$ & $?$ & $?$ & $3000.4 \pm 0.4$ & $4.5 \pm 0.7$ & $\Xi_{c} \bar{K}$ \\
\hline$\Omega_{c}(3050)^{0}$ & $?$ & $?$ & $?$ & $?$ & $?$ & $3050.2 \pm 0.33$ & $<1.2$ & $\Xi_{c} \bar{K}$ \\
\hline$\Omega_{c}(3065)^{0}$ & $?$ & $?$ & $?$ & $?$ & ? & $3065.6 \pm 0.4$ & $3.5 \pm 0.4$ & $\Xi_{c} \bar{K}$ \\
\hline$\Omega_{c}(3090)^{0}$ & $?$ & $?$ & $?$ & $?$ & $?$ & $3090.2 \pm 0.7$ & $8.7 \pm 1.3$ & $\Xi_{c}^{(\prime)} \bar{K}$ \\
\hline$\Omega_{c}(3119)^{0}$ & $?$ & $?$ & $?$ & $?$ & $?$ & $3119.1 \pm 1.0$ & $<2.6$ & $\Xi_{c}^{(\prime)} \bar{K}$ \\
\hline
\end{tabular}

given in Table IV are unmeasured. One has to rely on the quark model to determine the $J^{P}$ assignments.

In Table $\mathrm{V}$ configurations with $L_{k}+L_{K}=0,1,2$ for charmed baryons are shown. The quantum number assignments are from Tables I and IV. Only the $J^{P}=\frac{1}{2}+$ $\overline{\mathbf{3}}_{\mathbf{f}}$ multiplet with states: $\left(\Lambda_{b}^{0}, \Xi_{b}^{0}, \Xi_{b}^{-}\right)$, is established. Several multiplets are to be completed with the yet to be discovered states, such as $\Sigma_{b}^{0}, \Sigma_{b}^{* 0}, \Xi_{b}^{\prime}(5935)^{0}$ and so on. 
TABLE III. Allowed configurations with $L_{k}+L_{K}=0,1,2$ are shown. The angular momenta are defined as $\vec{S}_{[q q]} \equiv \vec{L}_{k}+\vec{S}_{q q}$, $\vec{J}_{l} \equiv \vec{S}_{[q q]}+\vec{L}_{K}$ and $\vec{J} \equiv \vec{J}_{l}+\vec{S}_{Q}$, which are the angular momenta of the diquark system, the light-degree of freedom and the whole baryon, respectively. The quantum number assignments are from Tables I and II, while those with (†) are taken from [4]. There are different assignments of the quantum number of $\Lambda_{c}(2940)$, see text for more details. There are plenty of states to be discovered.

\begin{tabular}{|c|c|c|c|c|c|c|c|c|}
\hline$n$ & $L_{K}$ & $L_{k}$ & flavor & $S_{q q}$ & $S_{[q q]}^{P}$ & $J_{l}^{P}$ & $J^{P}$ & $\mathcal{B}_{c}$ \\
\hline 1 & 0 & 0 & $\overline{\mathbf{3}}_{\mathrm{f}}$ & 0 & $0^{+}$ & $0^{+}$ & $\frac{1}{2}+$ & $\Lambda_{c}^{+}, \Xi_{c}^{+, 0}$ \\
\hline 2 & 0 & 0 & $\overline{\mathbf{3}}_{\mathrm{f}}$ & 0 & $0^{+}$ & $0^{+}$ & $\frac{1}{2}^{+}$ & $\Lambda_{c}(2765)^{+}(\dagger)$ \\
\hline 1 & 0 & 0 & $6_{\mathrm{f}}$ & 1 & $1^{+}$ & $1^{+}$ & $\frac{1}{2}^{+}$ & $\Sigma_{c}(2455)^{++,+, 0}, \Xi_{c}^{\prime+, 0}, \Omega_{c}^{0}$ \\
\hline 2 & 0 & 0 & $\mathbf{6}_{\mathrm{f}}$ & 1 & $1^{+}$ & $1^{+}$ & $\frac{1}{2}^{+}$ & $\Xi_{c}^{\prime}(2970)^{+, 0}(\dagger), \Omega_{c}(3090)^{0}(\dagger)$ \\
\hline 1 & 0 & 0 & $\mathbf{6}_{\mathrm{f}}$ & 1 & $1^{+}$ & $1^{+}$ & $\frac{3}{2}^{+}$ & $\Sigma_{c}(2520)^{++,+, 0}, \Xi_{c}(2645)^{+, 0}, \Omega_{c}(2770)^{0}$ \\
\hline 2 & 0 & 0 & $6_{\mathrm{f}}$ & 1 & $1^{+}$ & $1^{+}$ & $\frac{3}{2}^{+}$ & $\Omega_{c}(3119)^{0}(\dagger)$ \\
\hline 1 & 2 & 0 & $\overline{\mathbf{3}}_{\mathrm{f}}$ & 0 & $0^{+}$ & $2^{+}$ & $\frac{3}{2}^{+}$ & $\Lambda_{c}(2860)^{+}, \Xi_{c}(3055)^{+, 0}(\dagger)$ \\
\hline 1 & 2 & 0 & $\overline{\mathbf{3}}_{\mathrm{f}}$ & 0 & $0^{+}$ & $2^{+}$ & $\frac{5}{2}+$ & $\Lambda_{c}(2880)^{+}, \Xi_{c}(3080)^{+, 0}(\dagger)$ \\
\hline$n$ & 2 & 0 & $\mathbf{6}_{\mathrm{f}}$ & 1 & $1^{+}$ & $1^{+}$ & $\frac{1}{2}+, \frac{3}{2}^{+}$ & \\
\hline$n$ & 2 & 0 & $6_{\mathrm{f}}$ & 1 & $1^{+}$ & $2^{+}$ & $\frac{3}{2}^{2}, \frac{5}{2}^{+}$ & \\
\hline$n$ & 2 & 0 & $6_{\mathrm{f}}$ & 1 & $1^{+}$ & $3^{+}$ & $\frac{5}{2}+, \frac{7}{2}^{+}$ & \\
\hline$n$ & 0 & 2 & $\overline{\mathbf{3}}_{\mathrm{f}}$ & 0 & $2^{+}$ & $2^{+}$ & $\frac{3}{2}+, \frac{5}{2}+$ & \\
\hline$n$ & 0 & 2 & $6_{f}$ & 1 & $1^{+}$ & $1^{+}$ & $\frac{1}{2}+, \frac{3}{2}+$ & \\
\hline$n$ & 0 & 2 & $6_{f}$ & 1 & $2^{+}$ & $2^{+}$ & $\frac{3}{2}+, \frac{5}{2}+$ & \\
\hline$n$ & 0 & 2 & $6_{\mathrm{f}}$ & 1 & $3^{+}$ & $3^{+}$ & $\frac{5}{2}+\frac{7}{2}+$ & \\
\hline$n$ & 1 & 1 & $\overline{\mathbf{3}}_{\mathrm{f}}$ & 1 & $0^{-}$ & $1^{+}$ & $\frac{1}{2}+, \frac{3}{2}+$ & \\
\hline$n$ & 1 & 1 & $\overline{\mathbf{3}}_{\mathrm{f}}$ & 1 & $1^{-}$ & $0^{+}$ & $\frac{1}{2}+$ & \\
\hline$n$ & 1 & 1 & $\overline{\mathbf{3}}_{\mathrm{f}}$ & 1 & $1^{-}$ & $1^{+}$ & $\frac{1}{2}^{+}, \frac{3}{2}^{+}$ & \\
\hline$n$ & 1 & 1 & $\overline{\mathbf{3}}_{\mathrm{f}}$ & 1 & $1^{-}$ & $2^{+}$ & $\frac{3}{2}+, \frac{5}{2}+$ & \\
\hline$n$ & 1 & 1 & $\overline{\mathbf{3}}_{\mathrm{f}}$ & 1 & $2^{-}$ & $1^{+}$ & $\frac{1}{2}^{+}, \frac{3}{2}^{+}$ & \\
\hline$n$ & 1 & 1 & $\overline{\mathbf{3}}_{\mathrm{f}}$ & 1 & $2^{-}$ & $2^{+}$ & $\frac{3}{2}+, \frac{5}{2}^{+}$ & \\
\hline$n$ & 1 & 1 & $\overline{\mathbf{3}}_{\mathrm{f}}$ & 1 & $2^{-}$ & $3^{+}$ & $\frac{5}{2}+, \frac{7}{2}+$ & \\
\hline$n$ & 1 & 1 & $6_{f}$ & 0 & $1^{-}$ & $0^{+}$ & $\frac{1}{2}+$ & \\
\hline$n$ & 1 & 1 & $6_{f}$ & 0 & $1^{-}$ & $1^{+}$ & $\frac{1}{2}^{+}, \frac{3}{2}^{+}$ & \\
\hline$n$ & 1 & 1 & $6_{f}$ & 0 & $1^{-}$ & $2^{+}$ & $\frac{3}{2}+, \frac{5}{2}+$ & \\
\hline 1 & 1 & 0 & $\overline{\mathbf{3}}_{\mathrm{f}}$ & 0 & $0^{+}$ & $1^{-}$ & $\frac{1}{2}-$ & $\Lambda_{c}(2595)^{+}, \Xi_{c}(2790)^{+, 0}$ \\
\hline 2 & 1 & 0 & $\overline{\mathbf{3}}_{\mathrm{f}}$ & 0 & $0^{+}$ & $1^{-}$ & $\frac{1}{2}-$ & $\Lambda_{c}(2940)^{+}(\dagger)$ \\
\hline 1 & 1 & 0 & $\overline{\mathbf{3}}_{\mathrm{f}}$ & 0 & $0^{+}$ & $1^{-}$ & $\frac{3}{2}-$ & $\Lambda_{c}(2625)^{+}, \Xi_{c}(2815)^{+, 0}$ \\
\hline 2 & 1 & 0 & $\overline{\mathbf{3}}_{\mathrm{f}}$ & 0 & $0^{+}$ & $1^{-}$ & $\frac{3}{2}-$ & $\Lambda_{c}(2940)^{+}$ \\
\hline 1 & 1 & 0 & $6_{\mathrm{f}}$ & 1 & $1^{+}$ & $0^{-}$ & $\frac{1}{2}-$ & \\
\hline 1 & 1 & 0 & $6_{\mathrm{f}}$ & 1 & $1^{+}$ & $1^{-}$ & $\frac{1}{2}-\frac{3-}{2}-$ & \\
\hline 1 & 1 & 0 & $6_{f}$ & 1 & $1^{+}$ & $2^{-}$ & $\frac{3}{2}-$ & $\Sigma_{c}(2800)^{++,+, 0}(\dagger), \Xi_{c}^{\prime}(2930)^{+, 0}(\dagger), \Omega_{c}(3050)^{0}(\dagger)$ \\
\hline 1 & 1 & 0 & $\mathbf{6}_{\mathrm{f}}$ & 1 & $1^{+}$ & $2^{-}$ & $\frac{5}{2}-$ & $\Omega_{c}(3066)^{0}(\dagger)$ \\
\hline$n$ & 0 & 1 & $\overline{\mathbf{3}}_{\mathrm{f}}$ & 1 & $0^{-}$ & $0^{-}$ & $\frac{1}{2}-$ & \\
\hline$n$ & 0 & 1 & $\overline{\mathbf{3}}_{\mathrm{f}}$ & 1 & $1^{-}$ & $1^{-}$ & $\frac{1}{2}^{-}, \frac{3}{2}^{-}$ & \\
\hline$n$ & 0 & 1 & $\overline{\mathbf{3}}_{\mathrm{f}}$ & 1 & $2^{-}$ & $2^{-}$ & $\frac{3}{2}^{-}, \frac{5}{2}-$ & \\
\hline$n$ & 0 & 1 & $6_{f}$ & 0 & $1^{-}$ & $1^{-}$ & $\frac{1}{2}-, \frac{3}{2}-$ & \\
\hline
\end{tabular}

From Table $\mathrm{V}$ we see that there are plenty of states in the $L_{k}+L_{K}=0,1,2$ sector to be discovered.

As shown in Table IV, $\Lambda_{b}, \Xi_{b}^{0,-}$ and $\Omega_{b}$ are the few singly bottom baryons that decay weakly. We will study their decay modes in this work. In particular, $\Lambda_{b}^{0} \rightarrow \Lambda_{c}^{(* * *)} M$,
$\Xi_{b} \rightarrow \Xi_{c}^{(*, * *)} M$, and $\Omega_{b} \rightarrow \Omega_{c}^{(*, * *)} M$ decays with $M=\pi$, $K, \rho, K^{*}$ will be explored. In Table VI, we summery the transitions we are about to study. There are altogether 8 different $\mathcal{B}_{b} \rightarrow \mathcal{B}_{c}$ transitions, which can be classified into 3 types according to the quantum numbers of the initial and 
TABLE IV. Mass spectra and widths (in units of MeV) of bottom baryons. Experimental values are taken from the Particle Data Group [1], except those of $\Xi_{b}(6227)^{-}$, which are from [33].

\begin{tabular}{|c|c|c|c|c|c|c|c|c|}
\hline State & $J^{P}$ & $n$ & $\left(L_{K}, L_{k}\right)$ & $S_{[q q]}^{P}$ & $J_{\ell}^{P_{\ell}}$ & Mass & Width & Decay modes \\
\hline$\Lambda_{b}^{0}$ & $\frac{1}{2}^{+}$ & 1 & $(0,0)$ & $0^{+}$ & $0^{+}$ & $5619.60 \pm 0.17$ & & weak \\
\hline$\Lambda_{b}(5912)^{0}$ & $\frac{1}{2}-$ & 1 & $(1,0)$ & $0^{+}$ & $1^{-}$ & $5912.20 \pm 0.21$ & $<0.66$ & $\Lambda_{b} \pi \pi$ \\
\hline$\Lambda_{b}(5920)^{0}$ & $\frac{3}{2}-$ & 1 & $(1,0)$ & $0^{+}$ & $1^{-}$ & $5919.92 \pm 0.19$ & $<0.63$ & $\Lambda_{b}^{0} \pi \pi$ \\
\hline$\Sigma_{b}^{+}$ & $\frac{1}{2}^{+}$ & 1 & $(0,0)$ & $1^{+}$ & $1^{+}$ & $5811.3 \pm 1.9$ & $9.7_{-3.0}^{+4.0}$ & $\Lambda_{b} \pi$ \\
\hline$\Sigma_{b}^{-}$ & $\frac{1}{2}^{+}$ & 1 & $(0,0)$ & $1^{+}$ & $1^{+}$ & $5815.5 \pm 1.8$ & $4.9_{-2.4}^{+3.3}$ & $\Lambda_{b} \pi$ \\
\hline$\Sigma_{b}^{*+}$ & $\frac{3}{2}^{+}$ & 1 & $(0,0)$ & $1^{+}$ & $1^{+}$ & $5832.1 \pm 1.9$ & $11.5 \pm 2.8$ & $\Lambda_{b} \pi$ \\
\hline$\Sigma_{b}^{*-}$ & $\frac{3}{2}^{+}$ & 1 & $(0,0)$ & $1^{+}$ & $1^{+}$ & $5835.1 \pm 1.9$ & $7.5 \pm 2.3$ & $\Lambda_{b} \pi$ \\
\hline$\Xi_{b}^{0}$ & $\frac{1}{2}^{+}$ & 1 & $(0,0)$ & $0^{+}$ & $0^{+}$ & $5791.9 \pm 0.5$ & & weak \\
\hline$\Xi_{b}^{-}$ & $\frac{1}{2}^{+}$ & 1 & $(0,0)$ & $0^{+}$ & $0^{+}$ & $5794.5 \pm 1.4$ & & weak \\
\hline$\Xi_{b}^{\prime}(5935)^{-}$ & $\frac{1}{2}+$ & 1 & $(0,0)$ & $1^{+}$ & $1^{+}$ & $5935.02 \pm 0.05$ & $<0.08$ & $\Xi_{b}^{0} \pi^{-}$ \\
\hline$\Xi_{b}(5945)^{0}$ & $\frac{3}{2}+$ & 1 & $(0,0)$ & $1^{+}$ & $1^{+}$ & $5949.8 \pm 1.4$ & $0.90 \pm 0.18$ & $\Xi_{b} \pi$ \\
\hline$\Xi_{b}(5955)^{-}$ & $\frac{3}{2}+$ & 1 & $(0,0)$ & $1^{+}$ & $1^{+}$ & $5955.33 \pm 0.13$ & $1.65 \pm 0.33$ & $\Xi_{b} \pi$ \\
\hline$\Xi_{b}(6227)^{-}$ & $?$ & $?$ & $?$ & $?$ & $?$ & $6226.9 \pm 2.0 \pm 0.3 \pm 0.2$ & $18.1 \pm 5.4 \pm 1.8$ & $\Lambda_{b} K^{-}, \Xi_{b} \pi^{-}$ \\
\hline$\Omega_{b}^{0}$ & $\frac{1}{2}^{+}$ & 1 & $(0,0)$ & $1^{+}$ & $1^{+}$ & $6046.1 \pm 1.7$ & & weak \\
\hline
\end{tabular}

final state baryons. These three types of transitions are $\mathcal{B}_{b}\left(\overline{\mathbf{3}}_{\mathbf{f}}, 1 / 2^{+}\right)$to $\mathcal{B}_{c}\left(\overline{\mathbf{3}}_{\mathbf{f}}, 1 / 2^{+}\right), \quad \mathcal{B}_{b}\left(\mathbf{6}_{\mathbf{f}}, 1 / 2^{+}\right)$to $\mathcal{B}_{c}\left(\mathbf{6}_{\mathbf{f}}, 1 / 2^{+}\right)$, and $\mathcal{B}_{b}\left(\overline{\mathbf{3}}_{\mathbf{f}}, 1 / 2^{+}\right)$to $\mathcal{B}_{c}\left(\overline{\mathbf{3}}_{\mathbf{f}}, 1 / 2^{-}\right)$transitions. The type (i) and (iii) transitions have $\overline{\mathbf{3}}_{\mathbf{f}}$ scalar as spectators, while the type (ii) transition has $\mathbf{6}_{\mathbf{f}}$ axial-vector spectator diquarks. Among the final states three charmed baryons are denoted with $(\dagger)$, they are states with unspecified or ambiguous quantum numbers as noted in Tables II and III. As a working assumption we shall use the suggestion from Ref. [4] for their quantum numbers. Accordingly, we take $\Lambda_{c}(2765)$ as a radial excited $s$-wave state, $\Lambda_{c}(2940)$ a radial excited $p$-wave state and $\Omega_{c}(3090)$ a radial excited $s$-wave state. The study on these $\mathcal{B}_{b} \rightarrow \mathcal{B}_{c}$ transitions may shed light on the quantum numbers of these charmed baryons.

\section{FORM FACTORS IN THE LIGHT-FRONT APPROACH}

We consider a heavy baryon consisting a heavy quark $Q$ and a scalar isosinglet diquark $[q q]$ or an axial-vector isovector diquark $[q q]$. In the light-front approach, the baryon bound state with the total momentum $P$ and spin $J$ can be written as (see, e.g., [34,35])

$$
\begin{aligned}
\left|\mathcal{B}_{Q}\left(P, J, J_{z}\right)\right\rangle= & \int\left\{d^{3} p_{1}\right\}\left\{d^{3} p_{2}\right\} 2(2 \pi)^{3} \delta^{3}\left(\tilde{P}-\tilde{p}_{1}-\tilde{p}_{2}\right) \\
& \times \sum_{\lambda_{1}, m, \alpha-\epsilon, b-e} \Psi_{n L_{K} S_{[q q]} J_{l}}^{J J_{z}}\left(\tilde{p}_{1}, \tilde{p}_{2}, \lambda_{1}, \lambda_{2}\right) C_{\alpha \beta \gamma} F^{b c} \\
& \times\left|Q^{\alpha}\left(p_{1}, \lambda_{1}\right)\left[q_{b}^{\beta} q_{c}^{\gamma}\right]\left(p_{2}, \lambda_{2}\right)\right\rangle
\end{aligned}
$$

where $S_{[q q]}$ is the spin of the diquark, $L_{K}$ is the orbital angular momentum of the $Q-[q q]$ system, $J_{l}$ is the total angular momentum of the light degree of freedom, $n$ is the quantum number of the wave function (see later), $\alpha, \beta, \gamma$ and $b, c$ are color and flavor indices, respectively, $\lambda_{i}$ denotes helicity, $p_{1}$ and $p_{2}$ are the on-mass-shell lightfront momenta,

$\tilde{p}=\left(p^{+}, \vec{p}_{\perp}\right), \quad \vec{p}_{\perp}=\left(p^{1}, p^{2}\right), \quad p^{-}=\frac{m^{2}+p_{\perp}^{2}}{p^{+}}$

and

$\left\{d^{3} p\right\} \equiv \frac{d p^{+} d^{2} p_{\perp}}{2(2 \pi)^{3}}, \quad \delta^{3}(\tilde{p})=\delta\left(p^{+}\right) \delta^{2}\left(\vec{p}_{\perp}\right)$,

$\left|Q\left(p_{1}, \lambda_{1}\right)\left[q_{b} q_{c}\right]\left(p_{2}, \lambda_{2}\right)\right\rangle=b_{\lambda_{1}}^{\dagger}\left(p_{1}\right) a_{\lambda_{2}}^{\dagger}\left(p_{2}\right)|0\rangle$,

$\left[a_{\lambda^{\prime}}\left(p^{\prime}\right), a_{\lambda}^{\dagger}(p)\right]=2(2 \pi)^{3} \delta^{3}\left(\tilde{p}^{\prime}-\tilde{p}\right) \delta_{\lambda^{\prime}, \lambda}$,

$\left\{b_{\lambda^{\prime}}\left(p^{\prime}\right), b_{\lambda}^{\dagger}(p)\right\}=2(2 \pi)^{3} \delta^{3}\left(\tilde{p}^{\prime}-\tilde{p}\right) \delta_{\lambda^{\prime} \lambda}$,

with $\lambda_{2}=S_{2}=0$ for scalar diquark and $\lambda_{2}=0, \pm 1$ and $S_{2}=1$ for axial vector diquark. The coefficient $C_{\alpha \beta \gamma}$ is a normalized color factor and $F^{b c}$ is a normalized flavor coefficient, obeying the relation

$$
\begin{aligned}
C_{\alpha^{\prime} \beta^{\prime} \gamma^{\prime}} F^{b^{\prime} c^{\prime}} C_{\alpha \beta \gamma} F^{b c}\left\langle Q^{\alpha^{\prime}}\left(p_{1}^{\prime}, \lambda_{1}^{\prime}\right)\left[q_{b^{\prime}}^{\beta^{\prime}} q_{c^{\prime}}^{\gamma^{\prime}}\right]\left(p_{2}^{\prime}, \lambda_{2}^{\prime}\right)\right| \\
\left.\quad \times Q^{\alpha}\left(p_{1}, \lambda_{1}\right)\left[q_{a}^{\beta} q_{b}^{\gamma}\right]\left(p_{2}, \lambda_{2}\right)\right\rangle \\
=2^{2}(2 \pi)^{6} \delta^{3}\left(\tilde{p}_{1}^{\prime}-\tilde{p}_{1}\right) \delta^{3}\left(\tilde{p}_{2}^{\prime}-\tilde{p}_{2}\right) \delta_{\lambda_{1}^{\prime} \lambda_{1}} \delta_{\lambda_{2}^{\prime} \lambda_{2}} .
\end{aligned}
$$

The momenta can be defined in terms of the light-front relative momentum variables, $\left(x_{i}, \vec{k}_{i \perp}\right)$ for $i=1,2$, 
TABLE V. Same as Table III but for bottom baryons. The quantum number assignments are basically taken from Tables I and IV. There are plenty of states to be discovered.

\begin{tabular}{|c|c|c|c|c|c|c|c|c|}
\hline$n$ & $L_{K}$ & $L_{k}$ & flavor & $S_{q q}$ & $S_{[q q]}^{P}$ & $J_{l}^{P}$ & $J^{P}$ & $\mathcal{B}_{b}$ \\
\hline 1 & 0 & 0 & $\overline{\mathbf{3}}_{\mathrm{f}}$ & 0 & $0^{+}$ & $0^{+}$ & $\frac{1}{2}^{+}$ & $\Lambda_{b}^{0}, \Xi_{b}^{0,-}$ \\
\hline 1 & 0 & 0 & $6_{\mathrm{f}}$ & 1 & $1^{+}$ & $1^{+}$ & $\frac{1}{2}+$ & $\Sigma_{b}^{+,-}, \Xi_{b}^{\prime}(5935)^{-}, \Omega_{b}^{0}$ \\
\hline 1 & 0 & 0 & $\mathbf{6}_{\mathrm{f}}$ & 1 & $1^{+}$ & $1^{+}$ & $\frac{3}{2}+$ & $\Sigma_{b}^{*+,-}, \Xi_{b}(5945)^{+}, \Xi_{b}(5955)^{-}$ \\
\hline$n$ & 2 & 0 & $\overline{\mathbf{3}}_{\mathrm{f}}$ & 0 & $0^{+}$ & $2^{+}$ & $\frac{3}{2}^{+}, \frac{5}{2}+$ & \\
\hline$n$ & 2 & 0 & $6_{f}$ & 1 & $1^{+}$ & $1^{+}$ & $\frac{1}{2}^{+}, \frac{3}{2}+$ & \\
\hline$n$ & 2 & 0 & $6_{f}$ & 1 & $1^{+}$ & $2^{+}$ & $\frac{3}{2}^{+}, \frac{5}{2}+$ & \\
\hline$n$ & 2 & 0 & $6_{\mathrm{f}}$ & 1 & $1^{+}$ & $3^{+}$ & $\frac{5}{2}+, \frac{7}{2}+$ & \\
\hline$n$ & 0 & 2 & $\overline{\mathbf{3}}_{\mathrm{f}}$ & 0 & $2^{+}$ & $2^{+}$ & $\frac{3}{2}+, \frac{5}{2}+$ & \\
\hline$n$ & 0 & 2 & $6_{f}$ & 1 & $1^{+}$ & $1^{+}$ & $\frac{1}{2}+, \frac{3}{2}+$ & \\
\hline$n$ & 0 & 2 & $6_{\mathrm{f}}$ & 1 & $2^{+}$ & $2^{+}$ & $\frac{3}{2}^{+}, \frac{5}{2}+$ & \\
\hline$n$ & 0 & 2 & $6_{\mathrm{f}}$ & 1 & $3^{+}$ & $3^{+}$ & $\frac{5}{2}^{+}, \frac{7}{2}^{+}$ & \\
\hline$n$ & 1 & 1 & $\overline{\mathbf{3}}_{\mathrm{f}}$ & 1 & $0^{-}$ & $1^{+}$ & $\frac{1}{2}+, \frac{3}{2}+$ & \\
\hline$n$ & 1 & 1 & $\overline{\mathbf{3}}_{\mathrm{f}}$ & 1 & $1^{-}$ & $0^{+}$ & $\frac{1}{2}^{+}$ & \\
\hline$n$ & 1 & 1 & $\overline{\mathbf{3}}_{\mathrm{f}}$ & 1 & $1^{-}$ & $1^{+}$ & $\frac{1}{2}^{2}, \frac{3}{2}+$ & \\
\hline$n$ & 1 & 1 & $\overline{\mathbf{3}}_{\mathrm{f}}$ & 1 & $1^{-}$ & $2^{+}$ & $\frac{3}{2}+, \frac{5}{2}+$ & \\
\hline$n$ & 1 & 1 & $\overline{\mathbf{3}}_{\mathrm{f}}$ & 1 & $2^{-}$ & $1^{+}$ & $\frac{1}{2}+, \frac{3}{2}+$ & \\
\hline$n$ & 1 & 1 & $\overline{\mathbf{3}}_{\mathrm{f}}$ & 1 & $2^{-}$ & $2^{+}$ & $\frac{3}{2}+$ & \\
\hline$n$ & 1 & 1 & $\overline{\mathbf{3}}_{\mathrm{f}}$ & 1 & $2^{-}$ & $3^{+}$ & $\frac{5}{2}+, \frac{7}{2}+$ & \\
\hline$n$ & 1 & 1 & $6_{f}$ & 0 & $1^{-}$ & $0^{+}$ & 22 & \\
\hline$n$ & 1 & 1 & $6_{f}$ & 0 & $1^{-}$ & $1^{+}$ & $\frac{1}{2}^{+}, \frac{3}{2}+$ & \\
\hline$n$ & 1 & 1 & $6_{\mathrm{f}}$ & 0 & $1^{-}$ & $2^{+}$ & $\frac{3}{2}+, \frac{5}{2}+$ & \\
\hline 1 & 1 & 0 & $\overline{\mathbf{3}}_{\mathrm{f}}$ & 0 & $0^{+}$ & $1^{-}$ & & $\Lambda_{b}(5912)^{0}$ \\
\hline 1 & 1 & 0 & $\overline{\mathbf{3}}_{\mathrm{f}}$ & 0 & $0^{+}$ & $1^{-}$ & & $\Lambda_{b}(5920)^{0}$ \\
\hline$n$ & 0 & 1 & $\overline{\mathbf{3}}_{\mathrm{f}}$ & 1 & $0^{-}$ & $0^{-}$ & $\frac{1-}{2}$ & \\
\hline$n$ & 0 & 1 & $\overline{\mathbf{3}}_{\mathrm{f}}$ & 1 & $1^{-}$ & $1^{-}$ & $\frac{1-}{2}, \frac{3}{2}-$ & \\
\hline$n$ & 0 & 1 & $\overline{\mathbf{3}}_{\mathrm{f}}$ & 1 & $2^{-}$ & $2^{-}$ & $\frac{3}{2}-, \frac{5}{2}-$ & \\
\hline$n$ & 0 & 1 & $6_{f}$ & 0 & $1^{-}$ & $1^{-}$ & $\frac{1}{2}-, \frac{3}{2}-$ & \\
\hline$n$ & 1 & 0 & $6_{f}$ & 1 & $1^{+}$ & $0^{-}$ & $\frac{1}{2}^{-}$ & \\
\hline$n$ & 1 & 0 & $\mathbf{6}_{\mathrm{f}}$ & 1 & $1^{+}$ & $1^{-}$ & $\frac{1}{2}-, \frac{3}{2}-$ & \\
\hline$n$ & 1 & 0 & $6_{f}$ & 1 & $1^{+}$ & $2^{-}$ & $\frac{3}{2}-, \frac{5}{2}$ & \\
\hline
\end{tabular}

$$
\begin{aligned}
& p_{i}^{+}=x_{i} P^{+}, \quad \sum_{i=1}^{2} x_{i}=1, \\
& \vec{p}_{i \perp}=x_{i} \vec{P}_{\perp}+\vec{k}_{i \perp}, \quad \sum_{i=1}^{2} \vec{k}_{i \perp}=0 .
\end{aligned}
$$

The momentum-space wave-function $\Psi_{n L_{K} S_{[q]]} J_{l}}^{J J_{z}}$ can be expressed as

$$
\begin{aligned}
\Psi_{n L_{K}}^{J J_{z}} S_{[q q]} J_{l} & \left(\tilde{p}_{1}, \tilde{p}_{2}, \lambda_{1}, \lambda_{2}\right) \\
= & \left\langle\lambda_{1}\left|\mathcal{R}_{M}^{\dagger}\left(p_{1}^{+}, \vec{p}_{1 \perp}, m_{1}\right)\right| s_{1}\right\rangle\left\langle\lambda_{2}\left|\mathcal{R}_{M}^{\dagger}\left(p_{2}^{+}, \vec{p}_{2 \perp}, m_{2}\right)\right| s_{2}\right\rangle \\
& \times\left\langle S_{1} J_{l} ; s_{1} J_{l z} \mid S_{1} J_{l} ; J J_{z}\right\rangle\left\langle L_{K} S_{[q q]} ; L_{z} s_{2} \mid L_{k} S_{[q q]} ; J_{l} J_{l z}\right\rangle \\
& \times \phi_{n L_{K} L_{z}}\left(x_{1}, x_{2}, k_{1 \perp}, k_{2 \perp}\right),
\end{aligned}
$$

where $\phi_{n L_{K} L_{z}}\left(x_{1}, x_{2}, k_{1 \perp}, k_{2 \perp}\right)$ describes the momentum distribution of the constituents in the bound state, $\left\langle J^{\prime} J^{\prime \prime} ; m^{\prime} m^{\prime \prime} \mid J^{\prime} J^{\prime \prime} ; J m\right\rangle$ is the Clebsch-Gordan coefficients and $\left\langle\lambda_{i}\left|\mathcal{R}_{M}^{\dagger}\left(p_{1}^{+}, \vec{p}_{1 \perp}, m_{i}\right)\right| s_{i}\right\rangle$ is the well normalized Melosh transform matrix element. We will return to these quantities later.

We normalize the state as

$$
\begin{aligned}
& \left\langle\mathcal{B}_{Q}\left(P^{\prime}, J^{\prime}, J_{z}^{\prime}\right) \mid \mathcal{B}_{Q}\left(P, J, J_{z}\right)\right\rangle \\
& \quad=2(2 \pi)^{3} P^{+} \delta^{3}\left(\tilde{P}^{\prime}-\tilde{P}\right) \delta_{J^{\prime} J} \delta_{J_{z}^{\prime} J_{z}},
\end{aligned}
$$

consequently, $\phi_{n L L_{z}}\left(x, p_{\perp}\right)$ satisfies the following orthonormal condition, 
TABLE VI. Bottom baryon to charmed baryon transitions studied in this work are summarized in this table. There are three basic transition types. Type (i) is the $\mathcal{B}_{b}\left(\overline{\mathbf{3}}_{\mathbf{f}}, 1 / 2^{+}\right) \rightarrow \mathcal{B}_{c}\left(\overline{\mathbf{3}}_{\mathbf{f}}, 1 / 2^{+}\right)$transition, type (ii) is the $\mathcal{B}_{b}\left(\mathbf{6}_{\mathbf{f}}, 1 / 2^{+}\right) \rightarrow \mathcal{B}_{c}\left(\mathbf{6}_{\mathbf{f}}, 1 / 2^{+}\right)$transition and type (iii) is the $\mathcal{B}_{b}\left(\overline{\mathbf{3}}_{\mathbf{f}}, 1 / 2^{+}\right) \rightarrow \mathcal{B}_{c}\left(\overline{\mathbf{3}}_{\mathbf{f}}, 1 / 2^{-}\right)$transition. Note that type (i) and (iii) transitions involve scalar diquarks, while type (ii) transitions involve axial-vector diquarks. Type (iii) has odd parity baryons in the final states. The quantum number assignments are from Tables III and V, while those with $(\dagger)$ are taken from Ref. [4]. The asterisks indicate that the baryons in the final states are radial excited.

\begin{tabular}{lcc}
\hline \hline Type & $\left(n=1, L_{K}, S_{[q q]}^{P}, J_{l}^{P}, J^{P}\right)_{b} \rightarrow\left(n, L_{K}, S_{[q q]}^{P}, J_{l}^{P}, J^{P}\right)_{c}$ & $\mathcal{B}_{b} \rightarrow \mathcal{B}_{c}$ \\
\hline (i) & $\left(1,0,0^{+}, 0^{+}, \frac{1^{+}}{2}\right) \rightarrow\left(1,0,0^{+}, 0^{+}, \frac{1}{2}\right)$ & $\Lambda_{b}^{0} \rightarrow \Lambda_{c}^{+}, \Xi_{b}^{0(-)} \rightarrow \Xi_{c}^{+(0)}$ \\
(i)* & $\left(1,0,0^{+}, 0^{+}, \frac{1}{2}\right) \rightarrow\left(2,0,0^{+}, 0^{+}, \frac{1}{2}\right)$ & $\Lambda_{b}^{0} \rightarrow \Lambda_{c}(2765)^{+}(\dagger)$ \\
(ii) & $\left(1,0,1^{+}, 1^{+}, \frac{1}{2}^{+}\right) \rightarrow\left(1,0,1^{+}, 1^{+}, \frac{1}{2}\right)$ & $\Omega_{b}^{-} \rightarrow \Omega_{c}^{0}$ \\
(ii)* & $\left(1,0,1^{+}, 1^{+}, \frac{1}{2}+\right) \rightarrow\left(2,0,1^{+}, 1^{+}, \frac{1}{2}\right)$ & $\Omega_{b}^{-} \rightarrow \Omega_{c}(3090)^{0}(\dagger)$ \\
(iii) & $\left(1,0,0^{+}, 0^{+}, \frac{1}{2}^{+}\right) \rightarrow\left(1,1,0^{+}, 1^{-}, \frac{1}{2}\right)$ & $\Lambda_{b}^{0} \rightarrow \Lambda_{c}(2595)^{+}, \Xi_{b}^{0(-)} \rightarrow \Xi_{c}(2790)^{+(0)}$ \\
(iii)* & $\left(1,0,0^{+}, 0^{+}, \frac{1}{2}^{+}\right) \rightarrow\left(2,1,0^{+}, 1^{-}, \frac{1}{2}\right)$ & $\Lambda_{b}^{0} \rightarrow \Lambda_{c}(2940)^{+}(\dagger)$ \\
\hline \hline
\end{tabular}

$$
\int \frac{d x d^{2} p_{\perp}}{2(2 \pi)^{3}} \phi_{n^{\prime} L^{\prime} L_{z}^{\prime}}^{*}\left(x, p_{\perp}\right) \phi_{n L L_{z}}\left(x, p_{\perp}\right)=\delta_{n^{\prime}, n} \delta_{L^{\prime}, L} \delta_{L_{z}^{\prime}, L_{z}}
$$

The wave function is defined as

$$
\phi_{n L m}\left(\{x\},\left\{k_{\perp}\right\}\right)=\sqrt{\frac{d k_{2 z}}{d x_{2}}} \varphi_{n L m}\left(\frac{\vec{k}_{1}-\vec{k}_{2}}{2}, \beta\right)
$$

with

$$
\begin{aligned}
\varphi_{n 00}(\vec{k}, \beta) & =\varphi_{n s}(\vec{k}, \beta), \\
\varphi_{n 1 m}(\vec{k}, \beta) & =k_{m} \varphi_{n p}(\vec{k}, \beta) \\
& =-\varepsilon\left(k_{1}+k_{2}, m\right) \cdot k \varphi_{n p}(\vec{k}, \beta),
\end{aligned}
$$

where $k_{m} \equiv \vec{\varepsilon}(m) \cdot \vec{k}$ (or, explicitly $k_{L_{z}= \pm 1} \equiv \mp\left(k^{x} \pm i k^{y}\right) / \sqrt{2}$, $k_{L_{z}=0} \equiv k^{z}$ ) are proportional to the spherical harmonics $Y_{1 L_{z}}$ in momentum space, and $\varphi_{n s}$ and $\varphi_{n p}$ are the distribution amplitudes of $s$-wave and $p$-wave states, respectively. For a Gaussian-like wave function, one has (the first two are from Refs. [34,35])

$$
\begin{aligned}
& \varphi_{n=1, L_{K}=s}(\vec{k}, \beta)=4\left(\frac{\pi}{\beta^{2}}\right)^{\frac{3}{4}} \exp \left(-\frac{k_{z}^{2}+k_{\perp}^{2}}{2 \beta^{2}}\right), \\
& \varphi_{n=1, L_{K}=p}(\vec{k}, \beta)=\sqrt{\frac{2}{\beta^{2}} \varphi_{n=1}(\vec{k}, \beta),} \\
& \varphi_{n=2, L_{K}=s}(\vec{k}, \beta)=\sqrt{\frac{3}{2}}\left(1-\frac{2}{3} \frac{\vec{k}^{2}}{\beta^{2}}\right) \varphi_{n=1}(\vec{k}, \beta), \\
& \varphi_{n=2, L_{K}=p}(\vec{k}, \beta)=\sqrt{\frac{5}{2}}\left(1-\frac{2}{5} \frac{\vec{k}^{2}}{\beta^{2}}\right) \varphi_{n=1, L_{K}=p}(\vec{k}, \beta) .
\end{aligned}
$$

The kinematics are given by

$$
\begin{aligned}
M_{0}^{(\prime) 2} & =\sum_{i=1}^{2} \frac{m_{i}^{(\prime) 2}+k_{i \perp}^{(\prime) 2}}{x_{i}}, \\
k_{i}^{(\prime)} & =\left(\frac{m_{i}^{(\prime) 2}+k_{i \perp}^{(\prime) 2}}{x_{i}^{(\prime)} M_{0}^{(\prime)}}, x_{i}^{(\prime)} M_{0}^{(\prime)}, \vec{k}_{i \perp}^{(\prime)}\right) \\
& =\left(e_{i}^{(\prime)}-k_{i z}^{(\prime)}, e_{i}^{(\prime)}+k_{i z}^{(\prime)}, \vec{k}_{i \perp}^{(\prime)}\right), \\
M_{0}^{(\prime)} & =e_{1}^{(\prime)}+e_{2}^{((\prime)}, \\
e_{i}^{(\prime)} & =\sqrt{m_{i}^{(\prime) 2}+k_{i \perp}^{(\prime) 2}+k_{i z}^{(\prime) 2}}=\frac{x_{i}^{(\prime)} M_{0}^{(\prime)}}{2}+\frac{m_{i}^{(\prime) 2}+k_{i \perp}^{(\prime) 2}}{2 x_{i}^{(\prime)} M_{0}^{(\prime)}}, \\
k_{i z}^{(\prime)} & =\frac{x_{i}^{(\prime)} M_{0}^{(\prime)}}{2}-\frac{m_{i}^{(\prime) 2}+k_{i \perp}^{(\prime) 2}}{2 x_{i}^{(\prime)} M_{0}^{(\prime)}}, \\
2 M_{0}^{(\prime)} & \left(e_{1(2)}^{(\prime)}+m_{1(2)}^{(\prime)}\right)=\left(M_{0}^{(\prime)}+m_{1(2)}^{(\prime)}\right)^{2}-m_{2(1)}^{(\prime) 2} .
\end{aligned}
$$

Under the constraint of $1-\sum_{i=1}^{2} x_{i}=\sum_{i=1}^{2}\left(k_{i}\right)_{x, y, z}=0$, we have

$$
\frac{d k_{2 z}}{d x_{2}}=\frac{e_{1} e_{2}}{x_{1} x_{2} M_{0}}=\frac{d k_{1 z}}{d x_{1}}
$$

Now we turn to the Melosh transform. For the heavy quark part, we have [36,37],

$$
\left\langle\lambda_{1}\left|\mathcal{R}_{M}^{\dagger}\left(p_{1}^{+}, \vec{p}_{1 \perp}, m_{1}\right)\right| s_{1}\right\rangle=\frac{\bar{u}\left(p_{1}, \lambda_{1}\right) u_{D}\left(p_{1}, s_{1}\right)}{2 m_{1}}
$$

with $u_{(D)}$, a Dirac spinor in the light-front (instant) form. For the diquark part, if it is a scalar diquark the Melosh transform is a trivial one, i.e.,

$$
\left\langle\lambda_{2}\left|\mathcal{R}_{M}^{\dagger}\left(p_{2}^{+}, \vec{p}_{2 \perp}, m_{2}\right)\right| s_{2}\right\rangle=1
$$

but if it is a axial vector diquark, the Melosh transform is more interesting, 


$$
\left\langle\lambda_{2}\left|\mathcal{R}_{M}^{\dagger}\left(p_{2}^{+}, \vec{p}_{2 \perp}, m_{2}\right)\right| s_{2}\right\rangle=-\varepsilon_{L F}^{*}\left(p_{2}, \lambda_{2}\right) \cdot \varepsilon_{I}\left(p_{2}, s_{2}\right),
$$

where $\varepsilon_{L F}$ and $\varepsilon_{I}$ are polarization vectors in light-front and instant forms, respectively. Note that we have $u_{D}(k, s)=$ $u(k, \lambda)\left\langle\lambda\left|\mathcal{R}_{M}^{\dagger}\right| s\right\rangle$ and $\varepsilon_{I}(k, s)=\varepsilon_{L F}(k, \lambda)\left\langle\lambda\left|\mathcal{R}_{M}^{\dagger}\right| s\right\rangle$. Consequently, the state $|Q(k, \lambda)\rangle\left\langle\lambda\left|\mathcal{R}_{M}^{\dagger}\right| s\right\rangle$ and $|[q q](k, \lambda)\rangle \times$ $\left\langle\lambda\left|\mathcal{R}_{M}^{\dagger}\right| s\right\rangle$ transforms like $|Q(k, s)\rangle$ and $|[q q](k, s)\rangle$, respectively, under rotation, i.e., their transformation do not depend on their momentum. A crucial feature of the light-front formulation of a bound state, such as the one shown in Eq. (12), is the frame-independence of the light-front wave function [36,38]. Namely, the hadron can be boosted to any (physical) $\left(P^{+}, P_{\perp}\right)$ without affecting the internal variables $\left(x_{i}, \vec{k}_{\perp i}\right)$ of the wave function, which is certainly not the case in the instant-form formulation.

In practice it is more convenient to use the covariant form for $\Psi_{n L_{K} S_{[q]]} J_{l}}^{1 / 2 J_{z}}$ :

$$
\begin{aligned}
\Psi_{n L_{K} S_{[q q]}^{1 / 2 J_{l}}}^{1}\left(\tilde{p}_{1}, \tilde{p}_{2}, \lambda_{1}, \lambda_{2}\right)= & \frac{1}{\sqrt{\left(M_{0}+m_{1}\right)^{2}-m_{2}^{2}}} \bar{u}\left(p_{1}, \lambda_{1}\right) \\
& \times \Gamma_{L_{K} S_{[q]]} J_{l} u\left(\bar{P}, J_{z}\right)} \\
& \times \phi_{n L_{K}}\left(x_{1}, x_{2}, k_{1 \perp}, k_{2 \perp}\right), \quad(28)
\end{aligned}
$$

with

$$
\begin{aligned}
\Gamma_{s 00} & =1, \\
\Gamma_{s 11} & =\frac{\gamma_{5}}{\sqrt{3}}\left(\phi_{L F}^{*}\left(p_{2}, \lambda_{2}\right)-\frac{M_{0}+m_{1}+m_{2}}{\bar{P} \cdot p_{2}+m_{2} M_{0}} \varepsilon_{L F}^{*}\left(p_{2}, \lambda_{2}\right) \cdot \bar{P}\right), \\
\Gamma_{p 01} & =\frac{\gamma_{5}}{2 \sqrt{3}}\left(\not \not_{1}-\not p_{2}-\frac{m_{1}^{2}-m_{2}^{2}}{M_{0}}\right)
\end{aligned}
$$

for baryon states with a $S_{2}=0$ or $S_{2}=1$ diquark. The derivation of the above results can be found in Appendix A. Note that $\Gamma_{s 00}$ agrees with the one in Ref. [25], while $\Gamma_{s 11}$ and $\Gamma_{p 01}$ are new results and $\Gamma_{s 11}$ is different from those in Refs. [26,29,39], which have $\Gamma_{s 11}$ proportional to $\gamma_{5} \phi_{L F}^{*}\left(p_{2}, \lambda_{2}\right)$, instead.

It should be remarked that in the conventional LF approach $\bar{P}=p_{1}+p_{2}$ is not equal to the baryon's fourmomentum as all constituents are on-shell and consequently $u\left(\bar{P}, S_{z}\right)$ is not equal to $u\left(P, S_{z}\right)$; they satisfy different equations of motions $\left(\not P-M_{0}\right) u\left(\bar{P}, S_{z}\right)=0$ and $(P-M) u\left(P, S_{z}\right)=0$. This is similar to the case of a vector meson bound state where the polarization vectors $\varepsilon\left(\bar{P}, S_{z}\right)$ and $\varepsilon\left(P, S_{z}\right)$ are different and satisfy different equations $\varepsilon\left(\bar{P}, S_{z}\right) \cdot \bar{P}=0$ and $\varepsilon\left(P, S_{z}\right) \cdot P=0$ [40]. Although $u\left(\bar{P}, S_{z}\right)$ is different than $u\left(P, S_{z}\right)$, they satisfy the relation

$$
\gamma^{+} u\left(\bar{P}, S_{z}\right)=\gamma^{+} u\left(P, S_{z}\right)
$$

followed from $\gamma^{+} \gamma^{+}=0, \bar{P}^{+}=P^{+}, \bar{P}_{\perp}=P_{\perp}$. This is again in analogy with the case of $\varepsilon(\bar{P}, \pm 1)=\varepsilon(P, \pm 1)$.

Note that the normalization of state, Eq. (18), implies

$$
\begin{aligned}
\delta_{J_{z}^{\prime}, J_{z}}= & \int \frac{d x_{2} d^{2} k_{2 \perp}}{2(2 \pi)^{3}} \frac{\phi_{n L_{K}}^{*}\left(\left\{x_{j}\right\} ;\left\{\vec{k}_{j \perp}\right\}\right) \phi_{n L_{K}}\left(\left\{x_{i}\right\} ;\left\{\vec{k}_{i \perp}\right\}\right)}{2 \sqrt{\bar{P} \cdot p_{1}+m_{1} M_{0}^{\prime}} \sqrt{\bar{P} \cdot p_{1}+m_{1} M_{0}}} \\
& \times \bar{u}\left(\bar{P}, J_{z}^{\prime}\right) \bar{\Gamma}_{L_{K} S_{[q q]} J_{l}\left(\not \phi_{1}+m_{1}\right) \Gamma_{L_{K} S_{[q q]} J_{l}} u\left(\bar{P}, J_{z}\right),}
\end{aligned}
$$

with $\bar{\Gamma}_{L_{K} S_{[q q]} J_{l}} \equiv \gamma_{0} \Gamma_{L_{K} S_{[q q]} J_{l}}^{\dagger} \gamma_{0}$. To verify it we note that the right-hand-side of Eq. (31) is a matrix element of a $2 \times 2$ Hermitian matrix. Hence, its value can be extracted by taking traces with unit and sigma matrices, giving

$$
\begin{aligned}
1= & \frac{1}{2} \int \frac{d x_{2} d^{2} k_{2 \perp}}{2(2 \pi)^{3}} \frac{\phi_{n L_{K}}^{*}\left(\left\{x_{j}\right\} ;\left\{\vec{k}_{j \perp}\right\}\right) \phi_{n L_{K}}\left(\left\{x_{i}\right\} ;\left\{\vec{k}_{i \perp}\right\}\right)}{2 \sqrt{\bar{P} \cdot p_{1}+m_{1} M_{0}^{\prime}} \sqrt{\bar{P} \cdot p_{1}+m_{1} M_{0}}} \\
& \left.\times \operatorname{Tr}\left[\overline{(P}+M_{0}\right) \bar{\Gamma}_{L_{K} S_{[q q]} J_{l}}\left(\not \phi_{1}+m_{1}\right) \Gamma_{L_{K} S_{[q q]} J_{l}}\right],
\end{aligned}
$$

and

$$
\begin{aligned}
& 0=\int \frac{d x_{2} d^{2} k_{2 \perp}}{2(2 \pi)^{3}} \frac{\phi_{n L_{K}}^{*}\left(\left\{x_{j}\right\} ;\left\{\vec{k}_{j \perp}\right\}\right) \phi_{n L_{K}}\left(\left\{x_{i}\right\} ;\left\{\vec{k}_{i \perp}\right\}\right)}{8 P^{+} \sqrt{\bar{P} \cdot p_{1}+m_{1} M_{0}^{\prime}} \sqrt{\bar{P} \cdot p_{1}+m_{1} M_{0}}} \\
& \left.\left.\times \operatorname{Tr}\left[\overline{(P}+M_{0}\right) \gamma^{+} \gamma_{5} \overline{(P}+M_{0}\right) \bar{\Gamma}_{L_{K} S_{[q q]} J_{l}}\left(\not \not r_{1}+m_{1}\right) \Gamma_{\left.L_{K} S_{[q]]} J_{l}\right]}\right], \\
& 0=\int \frac{d x_{2} d^{2} k_{2 \perp}}{2(2 \pi)^{3}} \frac{\phi_{n L_{K}}^{*}\left(\left\{x_{j}\right\} ;\left\{\vec{k}_{j \perp}\right\}\right) \phi_{n L_{K}}\left(\left\{x_{i}\right\} ;\left\{\vec{k}_{i \perp}\right\}\right)}{8 P^{+} \sqrt{\bar{P} \cdot p_{1}+m_{1} M_{0}^{\prime}} \sqrt{\bar{P} \cdot p_{1}+m_{1} M_{0}}} \\
& \left.\left.\times \operatorname{Tr}\left[\overline{(P}+M_{0}\right) \sigma^{i+} \gamma_{5} \overline{(P}+M_{0}\right) \bar{\Gamma}_{L_{K} S_{[q q]} J_{l}}\left(\not \gamma_{1}+m_{1}\right) \Gamma_{\left.L_{K} S_{[q]]} J_{l}\right]}\right],
\end{aligned}
$$

where we have made use of the following identities in the above equations,

$$
\begin{aligned}
& \frac{1}{2} \sum_{J_{z}, J_{z}^{\prime}} u\left(\bar{P}, J_{z}\right)\left(\sigma^{3}\right)_{J_{z} J_{z}^{\prime}} \bar{u}\left(\bar{P}, J_{z}^{\prime}\right) \\
& \left.\left.=\frac{1}{4 P^{+}} \overline{(P}+M_{0}\right) \gamma^{+} \gamma_{5} \overline{(P}+M_{0}\right), \\
& \frac{1}{2} \sum_{J_{z}, J_{z}^{\prime}} u\left(\bar{P}, J_{z}\right)\left(\sigma_{\perp}^{i}\right)_{J_{z} J_{z}^{\prime}} \bar{u}\left(\bar{P}, J_{z}^{\prime}\right) \\
& \left.\quad=\frac{i}{4 P^{+}} \overline{(P}+M_{0}\right) \sigma^{i+} \gamma_{5}\left(\not P+M_{0}^{\prime}\right) .
\end{aligned}
$$

Equations (32) and (33) are nontrivial requirements and we check that using $\Gamma_{s 00}, \Gamma_{s 11}$, and $\Gamma_{p 01}$ in Eq. (29) and $\phi_{n L_{K}}$ in Eqs. (21) and (22), the above relations are indeed satisfied. ${ }^{4}$

\footnotetext{
${ }^{4}$ Note that some authors used vertex functions that do not satisfy Eq. (32), while some authors employed some ad hoc additional normalization factors to the vertex functions in order to satisfy Eq. (32). In this work, Eqs. (32) and (33) are satisfied automatically.
} 


\section{A. $\mathcal{B}_{b}(1 / 2) \rightarrow \mathcal{B}_{c}(1 / 2)$ weak transitions, a general discussion}

The Feynman diagram for a typical $\mathcal{B}_{b} \rightarrow \mathcal{B}_{c}$ transition, is shown in Fig. 1. For the $\mathcal{B}_{b}\left(1 / 2^{+}\right) \rightarrow \mathcal{B}_{c}\left(1 / 2^{+}\right)$transition, the matrix element can be parametrized as

$$
\begin{aligned}
\left\langle\mathcal{B}_{c}\left(P^{\prime}, J_{z}^{\prime}\right)\left|\bar{c} \gamma_{\mu} b\right| \mathcal{B}_{b}\left(P, J_{z}\right)\right\rangle & =\bar{u}\left(P^{\prime}, J_{z}^{\prime}\right)\left[f_{1}^{V}\left(q^{2}\right) \gamma_{\mu}+i \frac{f_{2}^{V}\left(q^{2}\right)}{M+M^{\prime}} \sigma_{\mu \nu} q^{\nu}+\frac{f_{3}^{V}\left(q^{2}\right)}{M+M^{\prime}} q_{\mu}\right] u\left(P, J_{z}\right), \\
\left\langle\mathcal{B}_{c}\left(P^{\prime}, J_{z}^{\prime}\right)\left|\bar{c} \gamma_{\mu} \gamma_{5} b\right| \mathcal{B}_{b}\left(P, J_{z}\right)\right\rangle & =\bar{u}\left(P^{\prime}, J_{z}^{\prime}\right)\left[g_{1}^{A}\left(q^{2}\right) \gamma_{\mu}+i \frac{g_{2}^{A}\left(q^{2}\right)}{M+M^{\prime}} \sigma_{\mu \nu} q^{\nu}+\frac{g_{3}^{A}\left(q^{2}\right)}{M+M^{\prime}} q_{\mu}\right] \gamma_{5} u\left(P, J_{z}\right),
\end{aligned}
$$

with $q=P-P^{\prime}$. For the $\mathcal{B}_{b}\left(1 / 2^{+}\right) \rightarrow \mathcal{B}_{c}\left(1 / 2^{-}\right)$transition, we have

$$
\begin{aligned}
\left\langle\mathcal{B}_{c}\left(P^{\prime}, J_{z}^{\prime}\right)\left|\bar{c} \gamma_{\mu} b\right| \mathcal{B}_{b}\left(P, J_{z}\right)\right\rangle & =\bar{u}\left(P^{\prime}, J_{z}^{\prime}\right)\left[g_{1}^{V}\left(q^{2}\right) \gamma_{\mu}+i \frac{g_{2}^{V}\left(q^{2}\right)}{M+M^{\prime}} \sigma_{\mu \nu} q^{\nu}+\frac{g_{3}^{V}\left(q^{2}\right)}{M+M^{\prime}} q_{\mu}\right] \gamma_{5} u\left(P, J_{z}\right), \\
\left\langle\mathcal{B}_{c}\left(P^{\prime}, J_{z}^{\prime}\right)\left|\bar{c} \gamma_{\mu} \gamma_{5} b\right| \mathcal{B}_{b}\left(P, J_{z}\right)\right\rangle & =\bar{u}\left(P^{\prime}, J_{z}^{\prime}\right)\left[f_{1}^{A}\left(q^{2}\right) \gamma_{\mu}+i \frac{f_{2}^{A}\left(q^{2}\right)}{M+M^{\prime}} \sigma_{\mu \nu} q^{\nu}+\frac{f_{3}^{A}\left(q^{2}\right)}{M+M^{\prime}} q_{\mu}\right] u\left(P, J_{z}\right) .
\end{aligned}
$$

Armed with the light-front quark model description of $\left|\mathcal{B}_{b}\left(P, J_{z}\right)\right\rangle$ in the previous subsection, we are ready to calculate the weak transition matrix element of heavy baryons. For a $\mathcal{B}_{b}(1 / 2) \rightarrow \mathcal{B}_{c}(1 / 2)$ transition, we have the general expressions

$$
\begin{aligned}
\left\langle\mathcal{B}_{c}\left(P^{\prime}, J_{z}^{\prime}\right)\left|\bar{c} \gamma^{\mu}\left(1-\gamma_{5}\right) b\right| \mathcal{B}_{b}\left(P, J_{z}\right)\right\rangle= & \int\left\{d^{3} p_{2}\right\} \frac{\phi_{n L_{K}^{\prime}}^{\prime *}\left(\left\{x^{\prime}\right\},\left\{k_{\perp}^{\prime}\right\}\right) \phi_{1 L_{K}}\left(\{x\},\left\{k_{\perp}\right\}\right)}{2 \sqrt{p_{1}^{+} p_{1}^{\prime+}\left(p_{1} \cdot \bar{P}+m_{1} M_{0}\right)\left(p_{1}^{\prime} \cdot \bar{P}^{\prime}+m_{1}^{\prime} M_{0}^{\prime}\right)}} \\
& \left.\times \bar{u}\left(\bar{P}^{\prime}, J_{z}^{\prime}\right) \bar{\Gamma}_{L_{K}^{\prime} S_{[q q]} J_{l}^{\prime}} \not p_{1}^{\prime}+m_{1}^{\prime}\right) \gamma^{\mu}\left(1-\gamma_{5}\right)\left(\not p_{1}+m_{1}\right) \Gamma_{L_{K} S_{[q q]} J_{l}} u\left(\bar{P}, J_{z}\right),
\end{aligned}
$$

where the diquark acts as an spectator and

$$
p_{i}^{(\prime)+}=x_{i}^{(\prime)} P^{(\prime)+}, \quad p_{i \perp}^{(\prime)}=x_{i}^{(\prime)} \vec{P}_{\perp}^{(\prime)}+\vec{k}_{i \perp}^{(\prime)}, \quad 1-\sum_{i=1}^{2} x_{i}^{(\prime)}=\sum_{i=1}^{2} \vec{k}_{i \perp}^{(\prime)}=0, \quad \tilde{p}_{1}-\tilde{p}_{1}^{\prime}=\tilde{q}, \quad \tilde{p}_{2}=\tilde{p}_{2}^{\prime},
$$

with $\Gamma_{L_{K} S_{[q q]} J_{l}}$ given in Eq. (29). As in $[15,34,41]$, we consider the $q^{+}=0, \vec{q}_{\perp} \neq \overrightarrow{0}$ case. We follow $[15,41]$ to

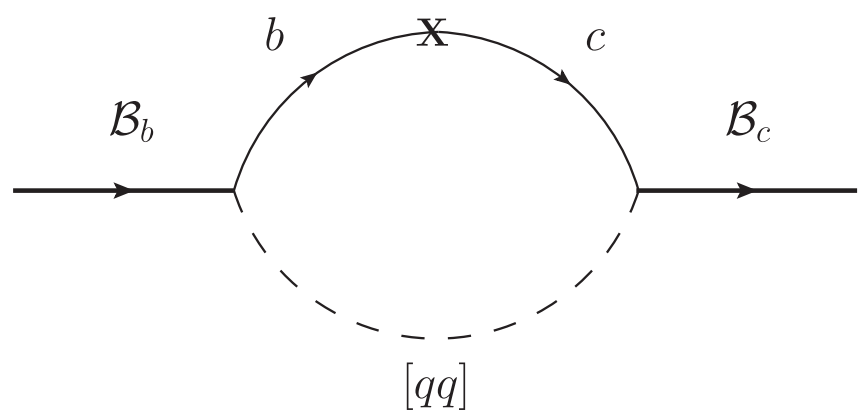

FIG. 1. Feynman diagram for a typical $\mathcal{B}_{b} \rightarrow \mathcal{B}_{c}$ transition, where the scalar or axial-vector diquark is denoted by a dashed line and the corresponding $V-A$ current vertex by $\mathrm{X}$. project out various form factors from the above transition matrix elements (see Appendix B for details). The results are given below.

\section{B. Form factors for $\mathcal{B}_{b}\left(\overline{\mathbf{3}}_{\mathrm{f}}, \mathbf{1} / \mathbf{2}^{+}\right) \rightarrow \mathcal{B}_{c}\left(\overline{\mathbf{3}}_{\mathrm{f}}, \mathbf{1} / \mathbf{2}^{+}\right)$ transition [type (i)]}

The $\mathcal{B}_{b}\left(\overline{\mathbf{3}}_{\mathbf{f}}, 1 / 2^{+}\right) \rightarrow \mathcal{B}_{c}\left(\overline{\mathbf{3}}_{\mathbf{f}}, 1 / 2^{+}\right)$transitions involve initial states in $\left(n, L_{K}, S_{[q q]}^{P}, J_{l}^{P}, J^{P}\right)_{b}=\left(1,0,0^{+}, 0^{+}, \frac{1}{2}{ }^{+}\right)$ configuration and final states in $\left(n, L_{K}, S_{[q q]}^{P}, J_{l}^{P}, J^{P}\right)_{c}=$ $\left(n, 0,0^{+}, 0^{+}, \frac{1}{2}\right)$ configurations (with $\left.n=1,2\right)$. Explicitly, we have $\Lambda_{b}^{0} \rightarrow \Lambda_{c}^{+}, \Xi_{b}^{0(-)} \rightarrow \Xi_{c}^{+(0)}$ and $\Lambda_{b}^{0} \rightarrow \Lambda_{c}(2765)^{+}$ transitions, where we follow Ref. [4] to take $\Lambda_{c}(2765)^{+}$as a radial excited $s$-wave state. In these transitions the scalar diquarks are spectators.

We obtain the following transition form factors for type (i) transition: 


$$
\begin{aligned}
f_{1}^{V}\left(q^{2}\right) & =\int \frac{d x_{2} d^{2} k_{2 \perp}}{2(2 \pi)^{3}} \frac{\phi_{n s}^{\prime *}\left(\left\{x^{\prime}\right\},\left\{k_{\perp}^{\prime}\right\}\right) \phi_{1 s}\left(\{x\},\left\{k_{\perp}\right\}\right)}{\sqrt{\left[\left(m_{1}+x_{1} M_{0}\right)^{2}+k_{1 \perp}^{2}\right]\left[\left(m_{1}^{\prime}+x_{1} M_{0}^{\prime}\right)^{2}+k_{1 \perp}^{\prime 2}\right]}} \times\left[k_{1 \perp} \cdot k_{1 \perp}^{\prime}+\left(m_{1}+x_{1} M_{0}\right)\left(m_{1}^{\prime}+x_{1}^{\prime} M_{0}^{\prime}\right)\right], \\
\frac{f_{2}^{V}\left(q^{2}\right)}{M+M^{\prime}} & =\frac{1}{\vec{q}_{\perp}^{2}} \int \frac{d x_{2} d^{2} k_{2 \perp}}{2(2 \pi)^{3}} \frac{\phi_{n s}^{\prime *}\left(\left\{x^{\prime}\right\},\left\{k_{\perp}^{\prime}\right\}\right) \phi_{1 s}\left(\{x\},\left\{k_{\perp}\right\}\right)}{\sqrt{\left[\left(m_{1}+x_{1} M_{0}\right)^{2}+k_{1 \perp}^{2}\right]\left[\left(m_{1}^{\prime}+x_{1} M_{0}^{\prime}\right)^{2}+k_{1 \perp}^{\prime 2}\right]}} \times\left[\left(m_{1}+x_{1} M_{0}\right) \vec{k}_{1 \perp}^{\prime} \cdot \vec{q}_{\perp}-\left(m_{1}^{\prime}+x_{1}^{\prime} M_{0}^{\prime}\right) \vec{k}_{1 \perp} \cdot \vec{q}_{\perp}\right], \\
g_{1}^{A}\left(q^{2}\right) & =\int \frac{d x_{2} d^{2} k_{2 \perp}}{2(2 \pi)^{3}} \frac{\phi_{n s}^{\prime *}\left(\left\{x^{\prime}\right\},\left\{k_{\perp}^{\prime}\right\}\right) \phi_{1 s}\left(\{x\},\left\{k_{\perp}\right\}\right)}{\sqrt{\left[\left(m_{1}+x_{1} M_{0}\right)^{2}+k_{1 \perp}^{2}\right]\left[\left(m_{1}^{\prime}+x_{1} M_{0}^{\prime}\right)^{2}+k_{1 \perp}^{\prime 2}\right]}} \times\left[-k_{1 \perp} \cdot k_{1 \perp}^{\prime}+\left(m_{1}+x_{1} M_{0}\right)\left(m_{1}^{\prime}+x_{1}^{\prime} M_{0}^{\prime}\right)\right], \\
\frac{g_{2}^{A}\left(q^{2}\right)}{M+M^{\prime}} & =\frac{1}{\vec{q}_{\perp}^{2}} \int \frac{d x_{2} d^{2} k_{2 \perp}}{2(2 \pi)^{3}} \frac{\phi_{n s}^{\prime *}\left(\left\{x^{\prime}\right\},\left\{k_{\perp}^{\prime}\right\}\right) \phi_{1 s}\left(\{x\},\left\{k_{\perp}\right\}\right)}{\sqrt{\left[\left(m_{1}+x_{1} M_{0}\right)^{2}+k_{1 \perp}^{2}\right]\left[\left(m_{1}^{\prime}+x_{1} M_{0}^{\prime}\right)^{2}+k_{1 \perp}^{\prime 2}\right]}} \times\left[\left(m_{1}^{\prime}+x_{1}^{\prime} M_{0}^{\prime}\right) \vec{k}_{1 \perp} \cdot \vec{q}_{\perp}+\left(m_{1}+x_{1} M_{0}\right) \vec{k}_{1 \perp}^{\prime} \cdot \vec{q}_{\perp}\right] .
\end{aligned}
$$

Note that we have $\vec{k}_{1 \perp}-\vec{k}_{1 \perp}^{\prime}=x_{2} \vec{q}_{\perp}$ and $q^{2}=-q_{\perp}^{2}$. For the transition with low laying final state $(n=1)$, the above equations are similar to those obtained in Ref. [15] and are identical to those in Ref. [25].

\section{Form factors for $\mathcal{B}_{b}\left(\mathbf{6}_{\mathrm{f}}, \mathbf{1} / \mathbf{2}^{+}\right) \rightarrow \mathcal{B}_{c}\left(\mathbf{6}_{\mathrm{f}}, \mathbf{1} / \mathbf{2}^{+}\right)$transition [type (ii)]}

The $\mathcal{B}_{b}\left(\mathbf{6}_{\mathbf{f}}, 1 / 2^{+}\right) \rightarrow \mathcal{B}_{c}\left(\mathbf{6}_{\mathbf{f}}, 1 / 2^{+}\right)$transitions involve initial states in $\left(n, L_{K}, S_{[q q]}^{P}, J_{l}^{P}, J^{P}\right)_{b}=\left(1,0,1^{+}, 1^{+}, \frac{1}{2}^{+}\right)$configuration and final states in $\left(n, L_{K}, S_{[q q]}^{P}, J_{l}^{P}, J^{P}\right)_{c}=\left(n, 0,1^{+}, 1^{+}, \frac{1}{2}{ }^{+}\right)$configurations (with $\left.n=1,2\right)$. Explicitly, we have $\Omega_{b}^{-} \rightarrow \Omega_{c}^{0}$ and $\Omega_{b}^{-} \rightarrow \Omega_{c}(3090)^{0}$ transitions, where we follow Ref. [4] to consider $\Omega_{c}(3090)^{0}$ as a radial excited $s$-wave state. In these transitions the axial-vector diquarks are spectators.

We obtain the following transition form factors for type (ii) transition:

$$
\begin{aligned}
f_{1}^{V}\left(q^{2}\right) & =\int \frac{d x_{2} d^{2} k_{2 \perp}}{2(2 \pi)^{3}} \frac{\phi_{n s}^{\prime *}\left(\left\{x^{\prime}\right\},\left\{k_{\perp}^{\prime}\right\}\right) \phi_{1 s}\left(\{x\},\left\{k_{\perp}\right\}\right)}{\sqrt{\left.\left[\left(m_{1}+x_{1} M_{0}\right)^{2}+k_{1 \perp}^{2}\right]\left[m_{1}^{\prime}+x_{1} M_{0}^{\prime}\right)^{2}+k_{1 \perp}^{\prime 2}\right]}} \times\left(A_{+}+B_{+}+C_{+}+D_{+}\right), \\
\frac{f_{2}^{V}\left(q^{2}\right)}{M+M^{\prime}} & =\frac{1}{\vec{q}_{\perp}^{2}} \int \frac{d x_{2} d^{2} k_{2 \perp}}{2(2 \pi)^{3}} \frac{\phi_{n s}^{\prime *}\left(\left\{x^{\prime}\right\},\left\{k_{\perp}^{\prime}\right\}\right) \phi_{1 s}\left(\{x\},\left\{k_{\perp}\right\}\right)}{\sqrt{\left[\left(m_{1}+x_{1} M_{0}\right)^{2}+k_{1 \perp}^{2}\right]\left[\left(m_{1}^{\prime}+x_{1} M_{0}^{\prime}\right)^{2}+k_{1 \perp}^{\prime 2}\right]}} \times\left(H_{+}+I_{+}+J_{+}+K_{+}\right), \\
g_{1}^{A}\left(q^{2}\right) & =\int \frac{d x_{2} d^{2} k_{2 \perp}}{2(2 \pi)^{3}} \frac{\phi_{n s}^{\prime *}\left(\left\{x^{\prime}\right\},\left\{k_{\perp}^{\prime}\right\}\right) \phi_{1 s}\left(\{x\},\left\{k_{\perp}\right\}\right)}{\sqrt{\left[\left(m_{1}+x_{1} M_{0}\right)^{2}+k_{1 \perp}^{2}\right]\left[\left(m_{1}^{\prime}+x_{1} M_{0}^{\prime}\right)^{2}+k_{1 \perp}^{\prime 2}\right]}} \times\left(A_{-}+B_{-}+C_{-}+D_{-}\right), \\
\frac{g_{2}^{A}\left(q^{2}\right)}{M+M^{\prime}} & =\frac{1}{\vec{q}_{\perp}^{2}} \int \frac{d x_{2} d^{2} k_{2 \perp}}{2(2 \pi)^{3}} \frac{\phi_{n s}^{\prime *}\left(\left\{x^{\prime}\right\},\left\{k_{\perp}^{\prime}\right\}\right) \phi_{1 s}\left(\{x\},\left\{k_{\perp}\right\}\right)}{\sqrt{\left[\left(m_{1}+x_{1} M_{0}\right)^{2}+k_{1 \perp}^{2}\right]\left[\left(m_{1}^{\prime}+x_{1} M_{0}^{\prime}\right)^{2}+k_{1 \perp}^{\prime 2}\right]}} \times\left(H_{-}+I_{-}+J_{-}+K_{-}\right),
\end{aligned}
$$

where we have

$$
\begin{aligned}
A_{+}= & \frac{2 e_{2} M_{0}}{6 m_{2}^{2}}\left[\left[4 e_{2}^{\prime} M_{0}^{\prime}+x_{2}\left(M_{0}^{\prime}+m_{1}^{\prime}\right)\left(M_{0}-2 M_{0}^{\prime}+m_{1}\right)+2 m_{2}^{2}\left(x_{2}-1\right)\right]\right. \\
& +2 x_{2}\left(e_{2}^{\prime} M_{0}^{\prime}\left[\left(M_{0}+m_{1}\right)\left(-2 M_{0}+M_{0}^{\prime}+m_{1}^{\prime}\right)+2 m_{2}^{2}\right]\right. \\
& \left.+m_{2}^{2}\left[M_{0}^{2}-M_{0}\left(4 M_{0}^{\prime}+m_{1}+2 m_{1}^{\prime}\right)+M_{0}^{\prime}\left(M_{0}^{\prime}-2 m_{1}-m_{1}^{\prime}\right)+2 q^{2}\right]\right) \\
& +2 m_{2}^{2}\left[-2 e_{2}^{\prime} M_{0}^{\prime}+m_{1}^{\prime}\left(M_{0}+2 M_{0}^{\prime}+m_{1}\right)+M_{0} M_{0}^{\prime}+2 M_{0} m_{1}+M_{0}^{\prime} m_{1}-m_{2}^{2}-2 q^{2}\right] \\
& \left.-x_{2}^{2}\left[\left(M_{0}-M_{0}^{\prime}\right)^{2}+q^{2}\right]\left(\left(M_{0}+m_{1}\right)\left(M_{0}^{\prime}+m_{1}^{\prime}\right)+2 m_{2}^{2}\right)\right],
\end{aligned}
$$




$$
\begin{aligned}
B_{+}= & \frac{1}{6 M_{0} m_{2}^{2}\left(e_{2}+m_{2}\right)}\left[-2 e_{2}^{2} M_{0}^{2}\left[4 e_{2}^{\prime} M_{0}^{\prime}+x_{2}\left(M_{0}^{\prime}+m_{1}^{\prime}\right)\left(M_{0}-2 M_{0}^{\prime}+m_{1}+m_{2}\right)\right]\right. \\
& +e_{2} M_{0}\left(-2 x_{2}\left\{e_{2}^{\prime} M_{0}^{\prime}\left(-2 M_{0}+M_{0}^{\prime}+m_{1}^{\prime}\right)\left(M_{0}+m_{1}+m_{2}\right)\right.\right. \\
& \left.+m_{2}\left[M_{0}^{2} m_{2}-M_{0} M_{0}^{\prime}\left(M_{0}^{\prime}+m_{1}^{\prime}+2 m_{2}\right)+M_{0}^{\prime}\left(M_{0}^{\prime}\left(m_{1}+2 m_{2}\right)+\left(m_{1}+m_{2}\right)\left(m_{1}^{\prime}-2 m_{2}\right)\right)+m_{2} q^{2}\right]\right\} \\
& \left.+4 m_{2}\left(e_{2}^{\prime} M_{0}^{\prime}\left(-M_{0}+m_{1}+2 m_{2}\right)+m_{2}\left(M_{0}^{2}-M_{0}^{\prime} m_{1}^{\prime}+q^{2}\right)\right)+x_{2}^{2}\left(M_{0}^{\prime}+m_{1}^{\prime}\right)\left(\left(M_{0}-M_{0}^{\prime}\right)^{2}+q^{2}\right)\left(M_{0}+m_{1}+m_{2}\right)\right) \\
& +m_{2}^{2}\left(2 e_{2}^{\prime} M_{0}^{\prime}\left\{M_{0}^{2}\left(-x_{2}\right)+M_{0}\left[M_{0}^{\prime}-x_{2}\left(m_{1}+m_{2}\right)+m_{1}^{\prime}+m_{2}\right]+\left(m_{1}+m_{2}\right)\left(M_{0}^{\prime}+m_{1}^{\prime}-m_{2}\right)\right\}\right. \\
& -2 m_{2}\left[-M_{0}^{3}+M_{0}^{2}\left(m_{1}+m_{2}\right)+M_{0} M_{0}^{\prime}\left(m_{1}^{\prime}+m_{2}\right)-M_{0} q^{2}+\left(m_{1}+m_{2}\right)\left(-M_{0}^{\prime} m_{1}^{\prime}+M_{0}^{\prime} m_{2}+q^{2}\right)\right] \\
& -x_{2}\left\{2 M_{0}^{4}-M_{0}^{3}\left(M_{0}^{\prime}-2 m_{1}+m_{1}^{\prime}-3 m_{2}\right)-M_{0}^{2}\left[M_{0}^{\prime}\left(m_{1}+2 m_{1}^{\prime}+m_{2}\right)+\left(m_{1}+m_{2}\right)\left(m_{1}^{\prime}+m_{2}\right)-2 q^{2}\right]\right. \\
& +M_{0} M_{0}^{\prime}\left[M_{0}^{\prime 2}+M_{0}^{\prime}\left(m_{1}^{\prime}+m_{2}\right)-2 m_{1}^{\prime}\left(m_{1}+m_{2}\right)\right]+M_{0} q^{2}\left(M_{0}^{\prime}+2 m_{1}+m_{1}^{\prime}+3 m_{2}\right) \\
& \left.\left.\left.+\left(M_{0}^{\prime 2}+q^{2}\right)\left(m_{1}+m_{2}\right)\left(M_{0}^{\prime}+m_{1}^{\prime}-m_{2}\right)\right\}+M_{0} x_{2}^{2}\left[\left(M_{0}-M_{0}^{\prime}\right)^{2}+q^{2}\right]\left(M_{0}+m_{1}+m_{2}\right)\right)\right]
\end{aligned}
$$

$$
\begin{aligned}
C_{+}= & \frac{1}{6 M_{0}^{\prime} m_{2}^{2}\left(e_{2}^{\prime}+m_{2}\right)}\left[2 e _ { 2 } M _ { 0 } \left(-4 e_{2}^{\prime 2} M_{0}^{\prime 2}+e_{2}^{\prime} M_{0}^{\prime}\left(2 m_{2}\left(-M_{0}^{\prime}+m_{1}^{\prime}+2 m_{2}\right)-x_{2}\left(M_{0}-2 M_{0}^{\prime}+m_{1}\right)\left(M_{0}^{\prime}+m_{1}^{\prime}+m_{2}\right)\right)\right.\right. \\
& \left.+m_{2}^{2}\left\{M_{0}\left(M_{0}^{\prime}+m_{1}^{\prime}+m_{2}\right)+M_{0}^{\prime 2}\left(-x_{2}\right)+M_{0}^{\prime}\left(m_{1}-x_{2}\left(m_{1}^{\prime}+m_{2}\right)+m_{2}\right)+\left(m_{1}-m_{2}\right)\left(m_{1}^{\prime}+m_{2}\right)\right\}\right) \\
& +x_{2}\left(-2 e_{2}^{\prime 2} M_{0}^{\prime 2}\left(M_{0}+m_{1}\right)\left(-2 M_{0}+M_{0}^{\prime}+m_{1}^{\prime}+m_{2}\right)-2 e_{2}^{\prime} M_{0}^{\prime} m_{2}\left[M_{0}^{2}\left(-M_{0}^{\prime}+m_{1}^{\prime}+2 m_{2}\right)-M_{0} M_{0}^{\prime}\left(m_{1}+2 m_{2}\right)\right.\right. \\
& \left.+M_{0}\left(m_{1}-2 m_{2}\right)\left(m_{1}^{\prime}+m_{2}\right)+m_{2}\left(M_{0}^{\prime 2}+q^{2}\right)\right]+m_{2}^{2}\left\{M_{0}^{3}\left(-M_{0}^{\prime}-m_{1}^{\prime}-m_{2}\right)-M_{0}^{2}\left(M_{0}^{\prime}\left(m_{1}+m_{2}\right)+\left(m_{1}-m_{2}\right)\left(m_{1}^{\prime}+m_{2}\right)\right)\right. \\
& +M_{0}\left(M_{0}^{\prime 2}+2 M_{0}^{\prime} m_{1}-q^{2}\right)\left(M_{0}^{\prime}+m_{1}^{\prime}+m_{2}\right)-q^{2}\left[2 M_{0}^{\prime 2}+M_{0}^{\prime}\left(m_{1}+2 m_{1}^{\prime}+3 m_{2}\right)+\left(m_{1}-m_{2}\right)\left(m_{1}^{\prime}+m_{2}\right)\right] \\
& \left.\left.+M_{0}^{\prime 2}\left(-2 M_{0}^{\prime 2}+M_{0}^{\prime}\left(m_{1}-2 m_{1}^{\prime}-3 m_{2}\right)+\left(m_{1}+m_{2}\right)\left(m_{1}^{\prime}+m_{2}\right)\right)\right\}\right) \\
& -2 m_{2}^{2}\left(m_{2}\left\{M_{0} M_{0}^{\prime}\left(m_{1}+m_{2}\right)+\left(m_{1}^{\prime}+m_{2}\right)\left(-M_{0} m_{1}+M_{0} m_{2}+q^{2}\right)-M_{0}^{\prime 3}+M_{0}^{\prime 2}\left(m_{1}^{\prime}+m_{2}\right)-M_{0}^{\prime} q^{2}\right\}\right. \\
& \left.\left.-2 e_{2}^{\prime} M_{0}^{\prime}\left(-M_{0} m_{1}+M_{0}^{\prime 2}+q^{2}\right)\right)+M_{0}^{\prime} x_{2}^{2}\left(\left(M_{0}-M_{0}^{\prime}\right)^{2}+q^{2}\right)\left(M_{0}^{\prime}+m_{1}^{\prime}+m_{2}\right)\left(e_{2}^{\prime}\left(M_{0}+m_{1}\right)+m_{2}^{2}\right)\right],
\end{aligned}
$$

$$
\begin{aligned}
D_{+}= & \frac{\left(m_{2}^{2}\left(M_{0}^{2}+M_{0}^{\prime 2}+q^{2}\right)-2 e_{2} e_{2}^{\prime} M_{0} M_{0}^{\prime}\right)}{12 M_{0} M_{0}^{\prime} m_{2}^{2}\left(e_{2}+m_{2}\right)\left(e_{2}^{\prime}+m_{2}\right)}\left[-2 x_{2}\left\{e_{2} M_{0}\left(M_{0}^{\prime}+m_{1}^{\prime}+m_{2}\right)\left(M_{0}-2 M_{0}^{\prime}+m_{1}+m_{2}\right)\right.\right. \\
& +m_{2}^{2}\left(M_{0}^{\prime}\left(e_{2}^{\prime}+M_{0}^{\prime}+m_{1}+m_{1}^{\prime}\right)+M_{0}^{2}+M_{0}\left(-2 M_{0}^{\prime}+m_{1}+m_{1}^{\prime}\right)\right) \\
& +m_{2}\left[e_{2}^{\prime} M_{0}^{\prime}\left(-M_{0}+M_{0}^{\prime}+m_{1}+m_{1}^{\prime}\right)+m_{1} m_{1}^{\prime}\left(M_{0}+M_{0}^{\prime}\right)+M_{0}^{\prime} m_{1}\left(M_{0}^{\prime}-M_{0}\right)+M_{0} m_{1}^{\prime}\left(M_{0}-M_{0}^{\prime}\right)-M_{0} M_{0}^{\prime}\left(M_{0}+M_{0}^{\prime}\right)\right] \\
& \left.+e_{2}^{\prime} M_{0}^{\prime}\left(M_{0}+m_{1}\right)\left(-2 M_{0}+M_{0}^{\prime}+m_{1}^{\prime}\right)+m_{2}^{3}\left(M_{0}+M_{0}^{\prime}\right)\right\} \\
& +4\left(e_{2} M_{0}\left(m_{2}\left(-M_{0}^{\prime}+m_{1}^{\prime}+m_{2}\right)-2 e_{2}^{\prime} M_{0}^{\prime}\right)+m_{2}\left(e_{2}^{\prime} M_{0}^{\prime}\left(-M_{0}+m_{1}+m_{2}\right)+M_{0} m_{2}\left(m_{1}^{\prime}+m_{2}\right)+M_{0}^{\prime} m_{2}\left(m_{1}+m_{2}\right)\right)\right) \\
& \left.+x_{2}^{2}\left(\left(M_{0}-M_{0}^{\prime}\right)^{2}+q^{2}\right)\left(M_{0}+m_{1}+m_{2}\right)\left(M_{0}^{\prime}+m_{1}^{\prime}+m_{2}\right)\right]
\end{aligned}
$$

$$
\begin{aligned}
D_{-}= & D_{+}+\frac{\left(2 e_{2} M_{0} e_{2}^{\prime} M_{0}^{\prime}-m_{2}^{2}\left(M_{0}^{2}+\left(M_{0}^{\prime}\right)^{2}+q^{2}\right)\right)}{6 M_{0} m_{2}^{2}\left(e_{2}+m_{2}\right) M_{0}^{\prime}\left(e_{2}^{\prime}+m_{2}\right)}\left(M_{0}+m_{1}+m_{2}\right)\left(M_{0}^{\prime}+m_{1}^{\prime}+m_{2}\right) \\
& \times\left\{x_{2}\left[-2 e_{2} M_{0}-2 e_{2}^{\prime} M_{0}^{\prime}+x_{2}\left(M_{0}^{2}+M_{0}^{\prime 2}+q^{2}\right)\right]+2 m_{2}^{2}\right\}
\end{aligned}
$$

$$
\begin{aligned}
H_{+}= & \frac{1}{3 m_{2}^{2}}\left[-2 e_{2} M_{0}\left(M_{0}^{\prime}+m_{1}^{\prime}\right)\left(\vec{k}_{2 \perp} \cdot \vec{q}_{\perp}-q^{2} x_{2}\right)+2 e_{2}^{\prime} \vec{k}_{2 \perp} \cdot \vec{q}_{\perp} M_{0}^{\prime}\left(M_{0}+m_{1}\right)\right. \\
& +x_{2}\left(\vec{k}_{2 \perp} \cdot \vec{q}_{\perp}\left(M_{0}-M_{0}^{\prime}\right)\left(\left(M_{0}+m_{1}\right)\left(M_{0}^{\prime}+m_{1}^{\prime}\right)+2 m_{2}^{2}\right)+m_{2}^{2} q^{2}\left(4 M_{0}+M_{0}^{\prime}+2 m_{1}+m_{1}^{\prime}\right)\right) \\
& +m_{2}^{2}\left(\vec{k}_{2 \perp} \cdot \vec{q}_{\perp}\left(-3 M_{0}+3 M_{0}^{\prime}-m_{1}+m_{1}^{\prime}\right)-2 q^{2}\left(M_{0}+M_{0}^{\prime}+m_{1}+m_{1}^{\prime}\right)\right) \\
& \left.-M_{0} q^{2} x_{2}^{2}\left(\left(M_{0}+m_{1}\right)\left(M_{0}^{\prime}+m_{1}^{\prime}\right)+2 m_{2}^{2}\right)\right]
\end{aligned}
$$




$$
\begin{aligned}
& I_{+}=\frac{1}{3 M_{0} m_{2}^{2}\left(e_{2}+m_{2}\right)}\left[\vec { k } _ { 2 \perp } \cdot \vec { q } _ { \perp } \left(2 e_{2}^{2} M_{0}^{2}\left(M_{0}^{\prime}+m_{1}^{\prime}\right)+e_{2} M_{0}\left\{-2 e_{2}^{\prime} M_{0}^{\prime}\left(M_{0}+m_{1}+m_{2}\right)\right.\right.\right. \\
& \left.+m_{2}\left(m_{2}\left(M_{0}-3 M_{0}^{\prime}+m_{1}-m_{1}^{\prime}\right)+\left(M_{0}-m_{1}\right)\left(M_{0}^{\prime}+m_{1}^{\prime}\right)+m_{2}^{2}\right)-x_{2}\left(M_{0}-M_{0}^{\prime}\right)\left(M_{0}^{\prime}+m_{1}^{\prime}\right)\left(M_{0}+m_{1}+m_{2}\right)\right\} \\
& +m_{2}^{2}\left\{M_{0}^{3}\left(1-x_{2}\right)+M_{0}^{2}\left[M_{0}^{\prime} x_{2}-x_{2}\left(m_{1}+m_{2}\right)+m_{1}+m_{2}\right]\right. \\
& \left.\left.+M_{0}\left[M_{0}^{\prime} x_{2}\left(m_{1}+m_{2}\right)-M_{0}^{\prime}\left(m_{1}^{\prime}+m_{2}\right)+q^{2}\right]+\left(m_{1}+m_{2}\right)\left(M_{0}^{\prime}\left(m_{2}-m_{1}^{\prime}\right)+q^{2}\right)\right\}\right) \\
& +q^{2}\left(-M_{0} x_{2}\left\{2 e_{2}^{2} M_{0}\left(M_{0}^{\prime}+m_{1}^{\prime}\right)+e_{2} m_{2}\left(M_{0}\left(M_{0}^{\prime}+m_{1}^{\prime}+2 m_{2}\right)-\left(m_{1}+m_{2}\right)\left(M_{0}^{\prime}+m_{1}^{\prime}-2 m_{2}\right)\right)\right.\right. \\
& \left.+m_{2}^{2}\left(M_{0}^{\prime}+m_{1}^{\prime}\right)\left(M_{0}+m_{1}+m_{2}\right)\right\}+M_{0}^{2} x_{2}^{2}\left(M_{0}+m_{1}+m_{2}\right)\left(e_{2}\left(M_{0}^{\prime}+m_{1}^{\prime}\right)+m_{2}^{2}\right) \\
& \left.\left.+m_{2}^{2}\left(2 e_{2} M_{0}\left(M_{0}^{\prime}+m_{1}^{\prime}\right)+M_{0} m_{2}\left(M_{0}^{\prime}+m_{1}^{\prime}+m_{2}\right)-m_{2}\left(m_{1}+m_{2}\right)\left(M_{0}^{\prime}+m_{1}^{\prime}-m_{2}\right)\right)\right)\right], \\
& J_{+}=\frac{1}{3 M_{0}^{\prime} m_{2}^{2}\left(e_{2}^{\prime}+m_{2}\right)}\left[\vec { k } _ { 2 \perp } \cdot \vec { q } _ { \perp } \left(2 e_{2} e_{2}^{\prime} M_{0} M_{0}^{\prime}\left(M_{0}^{\prime}+m_{1}^{\prime}+m_{2}\right)-2 e_{2}^{\prime 2} M_{0}^{\prime 2}\left(M_{0}+m_{1}\right)\right.\right. \\
& +e_{2}^{\prime} M_{0}^{\prime}\left\{x_{2}\left(M_{0}^{\prime}-M_{0}\right)\left(M_{0}+m_{1}\right)\left(M_{0}^{\prime}+m_{1}^{\prime}+m_{2}\right)\right. \\
& \left.+m_{2}\left[M_{0}\left(-M_{0}^{\prime}+m_{1}^{\prime}+3 m_{2}\right)-M_{0}^{\prime}\left(m_{1}+m_{2}\right)+\left(m_{1}-m_{2}\right)\left(m_{1}^{\prime}+m_{2}\right)\right]\right\} \\
& -m_{2}^{2}\left\{M_{0}^{\prime 2}\left[M_{0} x_{2}-x_{2}\left(m_{1}^{\prime}+m_{2}\right)+m_{1}^{\prime}+m_{2}\right]+M_{0}^{\prime}\left[-M_{0}\left(m_{1}+m_{2}\right)+M_{0} x_{2}\left(m_{1}^{\prime}+m_{2}\right)+q^{2}\right]\right. \\
& \left.\left.+\left(m_{1}^{\prime}+m_{2}\right)\left[M_{0}\left(m_{2}-m_{1}\right)+q^{2}\right]+M_{0}^{\prime 3}\left(1-x_{2}\right)\right\}\right) \\
& +q^{2}\left(e _ { 2 } ^ { \prime } M _ { 0 } ^ { \prime } \left\{-x_{2}\left[2 e_{2} M_{0}\left(M_{0}^{\prime}+m_{1}^{\prime}+m_{2}\right)+m_{2}^{2}\left(2 M_{0}+M_{0}^{\prime}+m_{1}^{\prime}+m_{2}\right)\right]\right.\right. \\
& \left.+M_{0} x_{2}^{2}\left(M_{0}+m_{1}\right)\left(M_{0}^{\prime}+m_{1}^{\prime}+m_{2}\right)+2 m_{2}^{2}\left(M_{0}+m_{1}\right)\right\} \\
& +m_{2}^{2}\left\{M_{0} x_{2}\left[m_{2}\left(-2 M_{0}^{\prime}-m_{1}+m_{1}^{\prime}\right)-\left(M_{0}^{\prime}+m_{1}\right)\left(M_{0}^{\prime}+m_{1}^{\prime}\right)+m_{2}^{2}\right]\right. \\
& +m_{2}^{2}\left(-M_{0}+M_{0}^{\prime}-m_{1}+m_{1}^{\prime}\right)+m_{2}\left(M_{0}+m_{1}\right)\left(M_{0}^{\prime}-m_{1}^{\prime}\right)+M_{0} M_{0}^{\prime} x_{2}^{2}\left(M_{0}^{\prime}+m_{1}^{\prime}+m_{2}\right) \\
& \left.\left.\left.+x_{2}\left(M_{0}^{\prime 2}-M_{0}^{\prime} m_{1}+q^{2}\right)\left(M_{0}^{\prime}+m_{1}^{\prime}+m_{2}\right)+m_{2}^{3}\right\}\right)\right] \\
& K_{+}=\frac{\left(m_{2}^{2}\left(M_{0}^{2}+M_{0}^{\prime 2}+q^{2}\right)-2 e_{2} e_{2}^{\prime} M_{0} M_{0}^{\prime}\right)}{6 M_{0} M_{0}^{\prime} m_{2}^{2}\left(e_{2}+m_{2}\right)\left(e_{2}^{\prime}+m_{2}\right)} \\
& \times\left[q^{2} x_{2}\left(M_{0}^{\prime}+m_{1}^{\prime}+m_{2}\right)\left[-2 e_{2} M_{0}+M_{0} x_{2}\left(M_{0}+m_{1}+m_{2}\right)+m_{2}\left(-M_{0}+m_{1}+m_{2}\right)\right]\right. \\
& -\vec{k}_{2 \perp} \cdot \vec{q}_{\perp}\left(-2 e_{2} M_{0}\left(M_{0}^{\prime}+m_{1}^{\prime}+m_{2}\right)+2 e_{2}^{\prime} M_{0}^{\prime}\left(M_{0}+m_{1}+m_{2}\right)+x_{2}\left(M_{0}-M_{0}^{\prime}\right)\left(M_{0}+m_{1}+m_{2}\right)\left(M_{0}^{\prime}+m_{1}^{\prime}+m_{2}\right)\right. \\
& \left.\left.-2 M_{0} m_{2}\left(m_{1}^{\prime}+m_{2}\right)+2 M_{0}^{\prime} m_{2}\left(m_{1}+m_{2}\right)\right)\right] \\
& K_{-}=K_{+}-\frac{\vec{k}_{2 \perp} \cdot \vec{q}_{\perp}\left(M_{0}+m_{1}+m_{2}\right)}{3 M_{0} M_{0}^{\prime} m_{2}^{2}\left(e_{2}+m_{2}\right)\left(e_{2}^{\prime}+m_{2}\right)}\left(m_{2}^{2}\left(M_{0}^{2}+\left(M_{0}^{\prime}\right)^{2}+q^{2}\right)-2 e_{2} e_{2}^{\prime} M_{0} M_{0}^{\prime}\right) \\
& \times\left[-2 e_{2}^{\prime} M_{0}^{\prime}+M_{0}^{\prime} x_{2}\left(M_{0}^{\prime}+m_{1}^{\prime}+m_{2}\right)+m_{2}\left(-M_{0}^{\prime}+m_{1}^{\prime}+m_{2}\right)\right]
\end{aligned}
$$

and $A_{-}$is equal to $A_{+}$, but with $M_{0}^{\prime} \rightarrow-M_{0}^{\prime}$ and $m_{1}^{\prime} \rightarrow-m_{1}^{\prime} ;\left(e_{2}+m_{2}\right) B_{-}$is equal to $\left(e_{2}+m_{2}\right) B_{+}$, but with $M_{0}^{\prime} \rightarrow-M_{0}^{\prime}, m_{1}^{\prime} \rightarrow-m_{1}^{\prime}$ and $e_{2}^{\prime} \rightarrow-e_{2}^{\prime} ;\left(e_{2}^{\prime}+m_{2}\right) C_{-}$ is equal to $-\left(e_{2}^{\prime}+m_{2}\right) C_{+}$, but with $M_{0}^{\prime} \rightarrow-M_{0}^{\prime}$, $m_{1}^{\prime} \rightarrow-m_{1}^{\prime}, e_{2}^{\prime} \rightarrow-e_{2}^{\prime}$ and $m_{2} \rightarrow-m_{2} ; H_{-}$is equal to $-H_{+}$, but with $M_{0}^{\prime} \rightarrow-M_{0}^{\prime}$ and $m_{1}^{\prime} \rightarrow-m_{1}^{\prime} ;\left(e_{2}+m_{2}\right) I_{-}$is equal to $-\left(e_{2}+m_{2}\right) I_{+}$, but with $M_{0}^{\prime} \rightarrow-M_{0}^{\prime}, m_{1}^{\prime} \rightarrow-m_{1}^{\prime}$ and $e_{2}^{\prime} \rightarrow-e_{2}^{\prime} ;\left(e_{2}^{\prime}+m_{2}\right) J_{-}$is equal to $\left(e_{2}^{\prime}+m_{2}\right) J_{+}$, but with $M_{0}^{\prime} \rightarrow-M_{0}^{\prime}, m_{1}^{\prime} \rightarrow-m_{1}^{\prime}, e_{2}^{\prime} \rightarrow-e_{2}^{\prime}$ and $m_{2} \rightarrow-m_{2}$. Note that we have $\vec{k}_{1 \perp}-\vec{k}_{1 \perp}^{\prime}=x_{2} \vec{q}_{\perp}$ and $q^{2}=-q_{\perp}^{2}$. The above formulas of the form factors are new results.

\section{Form factors for $\mathcal{B}_{b}\left(\overline{\mathbf{3}}_{\mathrm{f}}, \mathbf{1} / \mathbf{2}^{+}\right) \rightarrow \mathcal{B}_{c}\left(\overline{\mathbf{3}}_{\mathbf{f}}, \mathbf{1} / \mathbf{2}^{-}\right)$ transition [type (iii)]}

The $\mathcal{B}_{b}\left(\overline{\mathbf{3}}_{\mathbf{f}}, 1 / 2^{+}\right) \rightarrow \mathcal{B}_{c}\left(\overline{\mathbf{3}}_{\mathbf{f}}, 1 / 2^{-}\right)$transitions involve initial states in $\left(n, L_{K}, S_{[q q]}^{P}, J_{l}^{P}, J^{P}\right)_{b}=\left(1,0,0^{+}, 0^{+}, \frac{1}{2}+\right)$ configuration and final states in $\left(n, L_{K}, S_{[q q]}^{P}, J_{l}^{P}, J^{P}\right)_{c}=$ $\left(n, 1,0^{+}, 1^{-}, \frac{1-}{2}\right)$ configurations (with $\left.n=1,2\right)$. Explicitly, we have $\Lambda_{b}^{0} \rightarrow \Lambda_{c}(2595)^{+}, \quad \Xi_{b}^{0(-)} \rightarrow \Xi_{c}(2790)^{+(0)}$ and $\Lambda_{b}^{0} \rightarrow \Lambda_{c}(2940)^{+}$transitions, where we follow Ref. [4] to consider $\Lambda_{c}(2940)^{+}$as a radial excited $p$-wave state. In these transitions, the scalar diquarks are spectators. 
We obtain the following transition form factors for type (iii) transition:

$$
\begin{aligned}
f_{1}^{A}\left(q^{2}\right) & =\int \frac{d x_{2} d^{2} k_{2 \perp}}{2(2 \pi)^{3}} \frac{\phi_{n p}^{\prime *}\left(\left\{x^{\prime}\right\},\left\{k_{\perp}^{\prime}\right\}\right) \phi_{1 s}\left(\{x\},\left\{k_{\perp}\right\}\right) R_{+}}{\sqrt{\left[\left(m_{1}+x_{1} M_{0}\right)^{2}+k_{1 \perp}^{2}\right]\left[\left(m_{1}^{\prime}+x_{1} M_{0}^{\prime}\right)^{2}+k_{1 \perp}^{\prime 2}\right]}}, \\
\frac{f_{2}^{A}\left(q^{2}\right)}{M+M^{\prime}} & =\frac{1}{\vec{q}_{\perp}^{2}} \int \frac{d x_{2} d^{2} k_{2 \perp}}{2(2 \pi)^{3}} \frac{\phi_{n p}^{\prime *}\left(\left\{x^{\prime}\right\},\left\{k_{\perp}^{\prime}\right\}\right) \phi_{1 s}\left(\{x\},\left\{k_{\perp}\right\}\right) S_{+}}{\sqrt{\left[\left(m_{1}+x_{1} M_{0}\right)^{2}+k_{1 \perp}^{2}\right]\left[\left(m_{1}^{\prime}+x_{1} M_{0}^{\prime}\right)^{2}+k_{1 \perp}^{\prime 2}\right]}}, \\
g_{1}^{V}\left(q^{2}\right) & =\int \frac{d x_{2} d^{2} k_{2 \perp}}{2(2 \pi)^{3}} \frac{\phi_{n p}^{\prime *}\left(\left\{x^{\prime}\right\},\left\{k_{\perp}^{\prime}\right\}\right) \phi_{1 s}\left(\{x\},\left\{k_{\perp}\right\}\right) R_{-}}{\sqrt{\left[\left(m_{1}+x_{1} M_{0}\right)^{2}+k_{1 \perp}^{2}\right]\left[\left(m_{1}^{\prime}+x_{1} M_{0}^{\prime}\right)^{2}+k_{1 \perp}^{\prime 2}\right]}}, \\
\frac{g_{2}^{V}\left(q^{2}\right)}{M+M^{\prime}} & =\frac{1}{\vec{q}_{\perp}^{2}} \int \frac{d x_{2} d^{2} k_{2 \perp}}{2(2 \pi)^{3}} \frac{\phi_{n p}^{\prime *}\left(\left\{x^{\prime}\right\},\left\{k_{\perp}^{\prime}\right\}\right) \phi_{1 s}\left(\{x\},\left\{k_{\perp}\right\}\right) S_{-}}{\sqrt{\left[\left(m_{1}+x_{1} M_{0}\right)^{2}+k_{1 \perp}^{2}\right]\left[\left(m_{1}^{\prime}+x_{1} M_{0}^{\prime}\right)^{2}+k_{1 \perp}^{\prime 2}\right]}},
\end{aligned}
$$

where we have

$$
\begin{aligned}
R_{+}= & \frac{1}{4 \sqrt{3} M_{0}^{\prime}}\left[2 x _ { 2 } \left(\left(M_{0}^{\prime}+m_{1}^{\prime}\right)\left(e_{2} M_{0}\left(M_{0}^{\prime}+m_{1}^{\prime}\right)-M_{0}^{\prime} m_{1}^{\prime}\left(3 M_{0}+m_{1}\right)+M_{0} m_{1}^{\prime 2}+M_{0}^{\prime 2}\left(-m_{1}\right)\right)\right.\right. \\
& \left.+m_{2}^{2}\left(-e_{2} M_{0}+4 M_{0} M_{0}^{\prime}-M_{0} m_{1}^{\prime}+M_{0}^{\prime} m_{1}\right)\right)+2 e_{2}^{\prime} M_{0}^{\prime}\left(4 M_{0}^{\prime}\left(M_{0}+m_{1}\right)+x_{2}\left(-4 M_{0} M_{0}^{\prime}+\left(M_{0}^{\prime}+m_{1}^{\prime}\right)^{2}-m_{2}^{2}\right)\right) \\
& -2 m_{2}^{2}\left(-m_{1}^{\prime}\left(M_{0}-2 M_{0}^{\prime}+m_{1}\right)+M_{0}^{\prime}\left(3 M_{0}+M_{0}^{\prime}+3 m_{1}\right)+m_{1}^{\prime 2}\right)-2\left(M_{0}+m_{1}\right)\left(M_{0}^{\prime}-m_{1}^{\prime}\right)^{2}\left(M_{0}^{\prime}+m_{1}^{\prime}\right) \\
& \left.-x_{2}^{2}\left(\left(M_{0}-M_{0}^{\prime}\right)^{2}+q^{2}\right)\left(M_{0}^{\prime}+m_{1}^{\prime}-m_{2}\right)\left(M_{0}^{\prime}+m_{1}^{\prime}+m_{2}\right)+2 m_{2}^{4}\right], \\
S_{+}= & \frac{1}{2 \sqrt{3} M_{0}^{\prime}}\left[4 e_{2}^{\prime} \vec{k}_{2 \perp} \cdot \vec{q}_{\perp} M_{0}^{\prime 2}+\vec{k}_{2 \perp} \cdot \vec{q}_{\perp}\left(M_{0}\left(x_{2}-1\right)\left(M_{0}^{\prime 2}+2 M_{0}^{\prime} m_{1}^{\prime}+m_{1}^{\prime 2}-m_{2}^{2}\right)+M_{0}^{\prime 3}\left(-x_{2}-1\right)\right.\right. \\
& \left.+M_{0}^{\prime 2}\left(-m_{1}-2 m_{1}^{\prime} x_{2}+m_{1}^{\prime}\right)+M_{0}^{\prime}\left(-2 m_{1} m_{1}^{\prime}+m_{1}^{\prime 2}\left(1-x_{2}\right)+m_{2}^{2}\left(x_{2}-3\right)\right)-\left(m_{1}+m_{1}^{\prime}\right)\left(m_{1}^{\prime 2}-m_{2}^{2}\right)\right) \\
& \left.+q^{2} x_{2}\left(M_{0}\left(-x_{2}\right)+M_{0}+m_{1}\right)\left(M_{0}^{\prime 2}+2 M_{0}^{\prime} m_{1}^{\prime}+m_{1}^{\prime 2}-m_{2}^{2}\right)\right], \\
R_{-}= & \frac{1}{4 \sqrt{3} M_{0}^{\prime}}\left[-2 x_{2}\left(M_{0}^{\prime}+m_{1}^{\prime}\right)\left(e_{2} M_{0}\left(M_{0}^{\prime}+m_{1}^{\prime}\right)+M_{0}^{\prime} m_{1}^{\prime}\left(3 M_{0}+m_{1}\right)-M_{0} m_{1}^{\prime 2}+M_{0}^{\prime 2} m_{1}\right)\right. \\
& +2 m_{2}^{2} x_{2}\left(e_{2} M_{0}+4 M_{0} M_{0}^{\prime}-M_{0} m_{1}^{\prime}+M_{0}^{\prime} m_{1}\right)+2 e_{2}^{\prime} M_{0}^{\prime}\left(4 M_{0}^{\prime}\left(M_{0}+m_{1}\right)-x_{2}\left(4 M_{0} M_{0}^{\prime}+\left(M_{0}^{\prime}+m_{1}^{\prime}\right)^{2}-m_{2}^{2}\right)\right) \\
& +2 m_{2}^{2}\left(m_{1}^{\prime}\left(M_{0}+2 M_{0}^{\prime}+m_{1}\right)+M_{0}^{\prime}\left(-3 M_{0}+M_{0}^{\prime}-3 m_{1}\right)+m_{1}^{\prime 2}\right)-2\left(M_{0}+m_{1}\right)\left(M_{0}^{\prime}-m_{1}^{\prime}\right)^{2}\left(M_{0}^{\prime}+m_{1}^{\prime}\right) \\
& \left.+x_{2}^{2}\left(\left(M_{0}+M_{0}^{\prime}\right)^{2}+q^{2}\right)\left(M_{0}^{\prime}+m_{1}^{\prime}-m_{2}\right)\left(M_{0}^{\prime}+m_{1}^{\prime}+m_{2}\right)-2 m_{2}^{4}\right],
\end{aligned}
$$

and

$$
\begin{aligned}
S_{-}= & \frac{1}{2 \sqrt{3} M_{0}^{\prime}}\left[-4 e_{2}^{\prime} \vec{k}_{2 \perp} \cdot \vec{q}_{\perp} M_{0}^{\prime 2}+\vec{k}_{2 \perp} \cdot \vec{q}_{\perp}\left(M_{0}\left(x_{2}-1\right)\left(M_{0}^{\prime 2}+2 M_{0}^{\prime} m_{1}^{\prime}+m_{1}^{\prime 2}-m_{2}^{2}\right)\right.\right. \\
& \left.+M_{0}^{\prime 3}\left(x_{2}+1\right)-M_{0}^{\prime 2}\left(m_{1}-2 m_{1}^{\prime} x_{2}+m_{1}^{\prime}\right)-2 M_{0}^{\prime} m_{1} m_{1}^{\prime}+M_{0}^{\prime} m_{1}^{\prime 2}\left(x_{2}-1\right)-M_{0}^{\prime} m_{2}^{2}\left(x_{2}-3\right)-\left(m_{1}-m_{1}^{\prime}\right)\left(m_{1}^{\prime 2}-m_{2}^{2}\right)\right) \\
& \left.+q^{2} x_{2}\left(M_{0}\left(-x_{2}\right)+M_{0}+m_{1}\right)\left(M_{0}^{\prime 2}+2 M_{0}^{\prime} m_{1}^{\prime}+m_{1}^{\prime 2}-m_{2}^{2}\right)\right] .
\end{aligned}
$$

Note that we have $\vec{k}_{1 \perp}-\vec{k}_{1 \perp}^{\prime}=x_{2} \vec{q}_{\perp}$ and $q^{2}=-q_{\perp}^{2}$. The above formulas of the form factors are new results.

\section{NUMERICAL RESULTS}

In this section we will show the numerical results of various $\mathcal{B}_{b} \rightarrow \mathcal{B}_{c}$ transition form factors using formulas obtained in Sec. III. We then proceed to estimate the decay rates and up-down asymmetries of $\Lambda_{b} \rightarrow \Lambda_{c}^{(*, *)} M^{-}$, $\Xi_{b} \rightarrow \Xi_{c}^{(* *)} M^{-}$, and $\Omega_{b} \rightarrow \Omega_{c}^{(*)} M^{-}$decays using naïve factorization.

\section{A. $\mathcal{B}_{b} \rightarrow \mathcal{B}_{c}$ form factors}

The input parameters $m_{\left[q q^{\prime}\right]}, m_{q}, \beta$ are summarized in Table VII. The constituent quark and diquark masses are taken from Ref. [42]. For the diquark masses, we use $m_{[u d]}^{S}$ for $\Lambda_{b}$ and $\Lambda_{c}^{(*, *)}, m_{[u s]}^{S}$ for $\Xi_{b}$ and $\Xi_{c}^{(* *)}$, and $m_{[s s]}^{A}$ for $\Omega_{b}$ and $\Omega_{c}^{(*)}$. The $\beta$ s are chosen to reproduce the $\operatorname{Br}\left(\Lambda_{b} \rightarrow \Lambda_{c} P\right)$ data (see later discussion). 
TABLE VII. The input parameters $m_{\left[q q^{\prime}\right]}^{S, A}, m_{q}$ and $\beta$ 's (in units of $\mathrm{GeV}$ ) appearing in the Gaussian-type wave function (22). (The superscript $S$ and $A$ mean scalar and axial vector, respectively.) The constituent quark and diquark masses are taken from ref. [42].

\begin{tabular}{ccccccc}
\hline \hline$m_{[u d]}^{S}$ & $m_{[u s]}^{S}$ & $m_{[s s]}^{A}$ & $m_{b}$ & $m_{c}$ & $\beta_{b}$ & $\beta_{c}$ \\
\hline 0.710 & 0.948 & 1.203 & 4.88 & 1.55 & 0.72 & 0.37 \\
\hline \hline
\end{tabular}

The form factors of various $\mathcal{B}_{b} \rightarrow \mathcal{B}_{c}$ transitions can be obtained using formulas in the previous section. There are three types of transitions. For the type (i) transition, the $\mathcal{B}_{b}\left(\overline{\mathbf{3}}_{\mathbf{f}}, 1 / 2^{+}\right) \rightarrow \mathcal{B}_{c}\left(\overline{\mathbf{3}}_{\mathbf{f}}, 1 / 2^{+}\right)$transition, the form factors can be obtained by using Eq. (39), for the type (ii) transition, $\mathcal{B}_{b}\left(\mathbf{6}_{\mathbf{f}}, 1 / 2^{+}\right) \rightarrow \mathcal{B}_{c}\left(\mathbf{6}_{\mathbf{f}}, 1 / 2^{+}\right)$transition, we use Eq. (40) and for the type (iii) transition, the $\mathcal{B}_{b}\left(\overline{\mathbf{3}}_{\mathbf{f}}, 1 / 2^{+}\right) \rightarrow$ $\mathcal{B}_{c}\left(\overline{\mathbf{3}}_{\mathbf{f}}, 1 / 2^{-}\right)$transition, Eq. (51) should be employed.

As our calculation of form factors is done in the $q^{+}=0$ frame, where $q^{2} \leq 0$, we shall follow $[34,43,44]$ to analytically continue the form factors to the timelike region. We find that the momentum dependence of the form factors in the spacelike region can be well parametrized and reproduced in the three-parameter form:

$$
F\left(q^{2}\right)=\frac{F(0)}{\left(1-q^{2} / M^{2}\right)\left[1-a\left(q^{2} / M^{2}\right)+b\left(q^{2} / M^{2}\right)^{2}\right]}
$$

for $\mathcal{B}_{b} \rightarrow \mathcal{B}_{c}$ transitions. The parameters $a, b$ and $F(0)$ are first determined in the spacelike region. We then employ this parametrization to determine the physical form factors at $q^{2} \geq 0$. The parameters $a, b$ are expected to be of order $\mathcal{O}(1)$. As we shall see this is usually true in our numerical results. Occasionally some $a$ s and $b$ s are larger than $\mathcal{O}(1)$, but in most of these cases the corresponding form factors are small and do not have much impact on decay rates.

The $\mathcal{B}_{b}\left(\overline{\mathbf{3}}_{\mathbf{f}}, 1 / 2^{+}\right) \rightarrow \mathcal{B}_{c}\left(\overline{\mathbf{3}}_{\mathbf{f}}, 1 / 2^{+}\right)$transition form factors $f_{1,2}^{V}\left(q^{2}\right)$ and $g_{1,2}^{A}\left(q^{2}\right)$ are given in Table VIII and are plotted in Fig. 2. These include the form factors for

TABLE VIII. The transition form factors for various $\mathcal{B}_{b}\left(\overline{\mathbf{3}}_{\mathbf{f}}, 1 / 2^{+}\right) \rightarrow \mathcal{B}_{c}\left(\overline{\mathbf{3}}_{\mathbf{f}}, 1 / 2^{+}\right)$transitions [type (i)]. We use a three parameter form for these form factors, see Eq. (56).

\begin{tabular}{lcrcccccc}
\hline \hline $\mathcal{B}_{b} \rightarrow \mathcal{B}_{c}$ & $F$ & $F(0)$ & $a$ & $b$ & $F$ & $F(0)$ & $a$ & $b$ \\
\hline$\Lambda_{b} \rightarrow \Lambda_{c}$ & $f_{1}^{V}$ & 0.48 & 0.42 & 0.31 & $g_{1}^{A}$ & 0.47 & 0.40 & 0.32 \\
& $f_{2}^{V}$ & -0.05 & 1.02 & 0.64 & $g_{2}^{A}$ & -0.14 & 0.77 & 0.50 \\
$\Lambda_{b} \rightarrow \Lambda_{c}(2765)$ & $f_{1}^{V}$ & 0.34 & 0.57 & 0.58 & $g_{1}^{A}$ & 0.33 & 0.55 & 0.58 \\
& $f_{2}^{V}$ & -0.07 & 1.07 & 0.84 & $g_{2}^{A}$ & -0.10 & 0.57 & 0.87 \\
$\Xi_{b} \rightarrow \Xi_{c}$ & $f_{1}^{V}$ & 0.40 & 1.02 & 0.84 & $g_{1}^{A}$ & 0.39 & 0.99 & 0.82 \\
& $f_{2}^{V}$ & -0.05 & 1.58 & 1.67 & $g_{2}^{A}-0.14$ & 1.36 & 1.34 \\
\hline \hline
\end{tabular}

$\Lambda_{b} \rightarrow \Lambda_{c}, \Lambda_{c}(2765)$, and $\Xi_{b} \rightarrow \Xi_{c}$ transitions. In this case, we have $f_{1}^{V}, g_{1}^{A}>0$ and $f_{2}^{V}, g_{2}^{A}<0$. We see that $\left|f_{1}^{V}\right|$ and $\left|g_{1}^{A}\right|$ are larger than $\left|f_{2}^{V}\right|$ and $\left|g_{2}^{A}\right|$ in these transitions. Note that except $\left|f_{2}^{V}\right|$, the $\Lambda_{b} \rightarrow \Lambda_{c}(2765)$ transition form factors have smaller sizes comparing to those in the other two transitions. This is reasonable, since $\Lambda_{c}(2765)$ is a radial excited state. The configurations of the final states in excited state differ from those in the low lying states and larger mismatch between initial and final state configurations, usually lead to smaller form factors.

The $\mathcal{B}_{b}\left(\mathbf{6}_{\mathbf{f}}, 1 / 2^{+}\right) \rightarrow \mathcal{B}_{c}\left(\mathbf{6}_{\mathbf{f}}, 1 / 2^{+}\right)$transition form factors $f_{1,2}^{V}\left(q^{2}\right)$ and $g_{1,2}^{A}\left(q^{2}\right)$ are given in Table IX and are plotted in Fig. 3. These includes the form factors for $\Omega_{b} \rightarrow \Omega_{c}$ and $\Omega_{c}$ (3090) transitions. In this case, we have $f_{1}^{V}, f_{2}^{V}>0, g_{1}^{A}$, and $g_{2}^{A}<0$. We see that $\left|f_{1}^{V}\right|$ and $\left|f_{2}^{V}\right|$ are larger than $\left|g_{1}^{A}\right|$ and $\left|g_{2}^{A}\right|$ in these transitions. Note that except $g_{2}^{A}$, the $\Omega_{b} \rightarrow \Omega_{c}$ (2940) transition form factors have smaller sizes comparing to those in the $\Omega_{b} \rightarrow \Omega_{c}$ transition. This is reasonable, since we take $\Omega_{c}(2940)$ as a radial excited state. Larger mismatch between initial and final state configurations, usually lead to smaller form factors.

The $\mathcal{B}_{b}\left(\overline{\mathbf{3}}_{\mathbf{f}}, 1 / 2^{+}\right) \rightarrow \mathcal{B}_{c}\left(\overline{\mathbf{3}}_{\mathbf{f}}, 1 / 2^{-}\right)$transition form factors $f_{1,2}^{A}\left(q^{2}\right)$ and $g_{1,2}^{V}\left(q^{2}\right)$ are given in Table $\mathrm{X}$ and are plotted in Fig. 4. These includes the form factors for $\Lambda_{b} \rightarrow \Lambda_{c}(2595), \Lambda_{c}(2940)$, and $\Xi_{b} \rightarrow \Xi_{c}(2790)$ transitions. In this case, we have $f_{1}^{A}, g_{1}^{V}>0$ and $f_{2}^{A}, g_{2}^{V}<0$. The signs of the form factors are identical to those in the $\mathcal{B}_{b}\left(\overline{\mathbf{3}}_{\mathbf{f}}, 1 / 2^{+}\right) \rightarrow \mathcal{B}_{c}\left(\overline{\mathbf{3}}_{\mathbf{f}}, 1 / 2^{+}\right)$case. The transitions in this case have $p$-wave final state baryons. In the previous two cases, the initial and final state baryons belong to the same categories $\left[\mathcal{B}_{b}\left(\overline{\mathbf{3}}_{\mathbf{f}}, 1 / 2^{+}\right)\right.$or $\left.\mathcal{B}_{c}\left(\mathbf{6}_{\mathbf{f}}, 1 / 2^{+}\right)\right]$, while in this case they are in different categories, the initial state is a $s$-wave baryon, but the final state is a $p$-wave baryon. We see that some of these form factors behavior rather differently from the previous ones. For example, as shown in Fig. 4, $f_{1}^{A}\left(q^{2}\right)$ are almost independent of $q^{2}$, which are different from the $f_{1}^{V}\left(q^{2}\right)$ in the previous cases. Furthermore, all four form factors are of similar sizes in this case, while in the previous cases either one or two form factors are much smaller than the others. Note that the transition form factors of $\Lambda_{b} \rightarrow \Lambda_{c}$ (2940) are similar to those in $\Lambda_{b} \rightarrow \Lambda_{c}$ (2595), even though $\Lambda_{c}(2940)$ is a radial excited $p$-wave state. This feature is also different from the two previous cases, where form factors involving radial excited states are usually smaller in sizes.

\section{B. $\mathcal{B}_{b} \rightarrow \mathcal{B}_{c} M$ decay rates and up-down asymmetries}

Under the factorization approximation, the decay amplitudes for color-allowed $\mathcal{B}_{b} \rightarrow \mathcal{B}_{c} M^{-}$decays are given by 


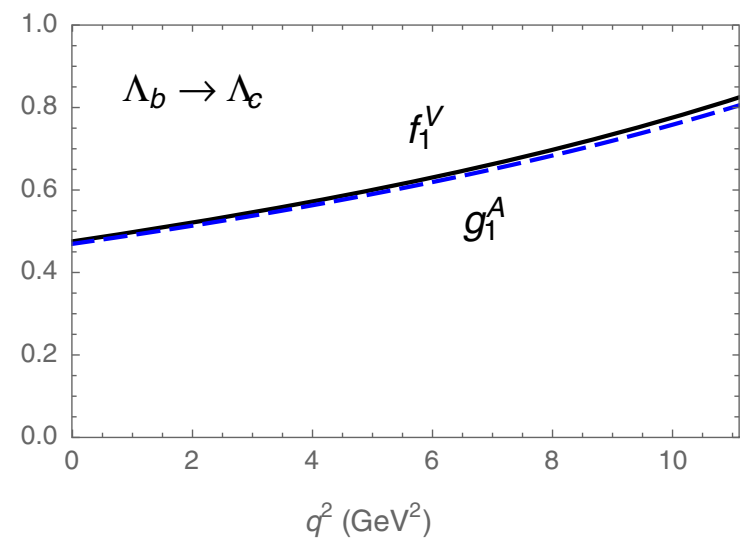

(a)

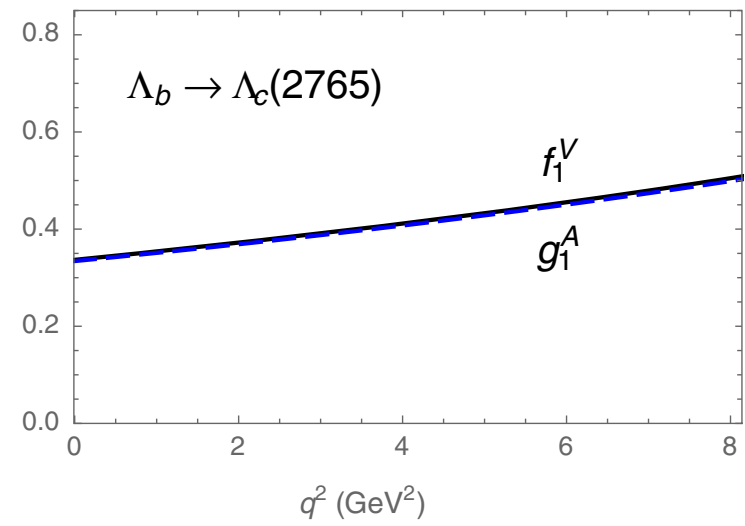

(c)

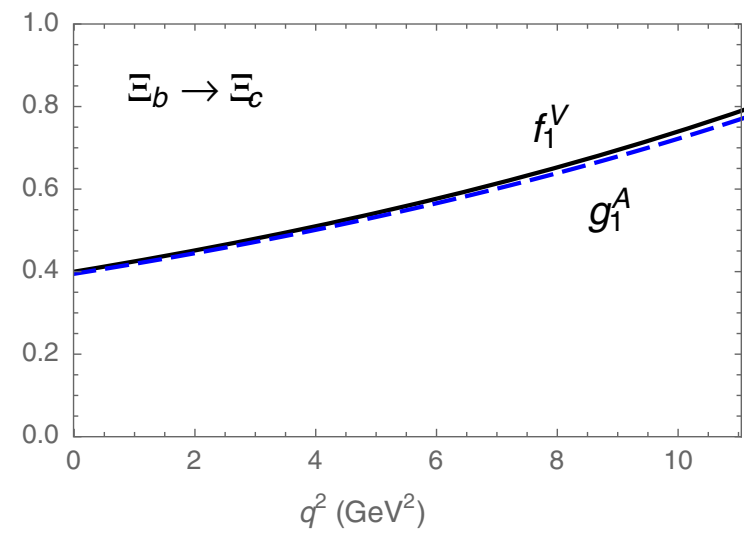

(e)

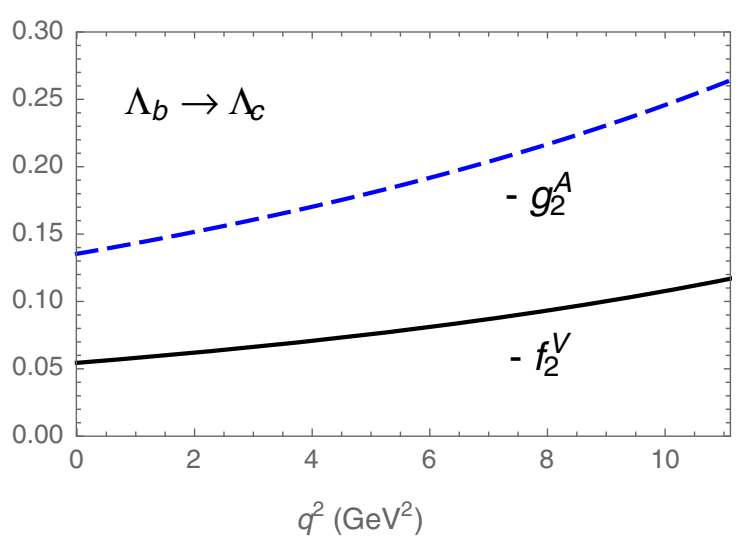

(b)

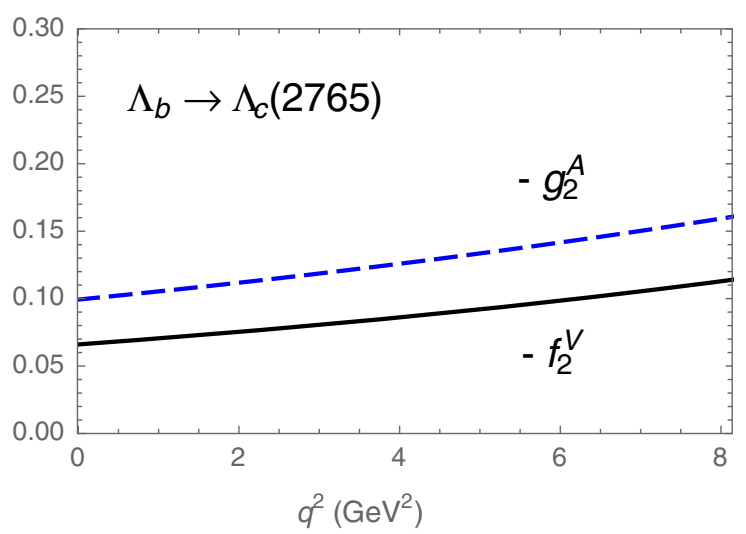

(d)

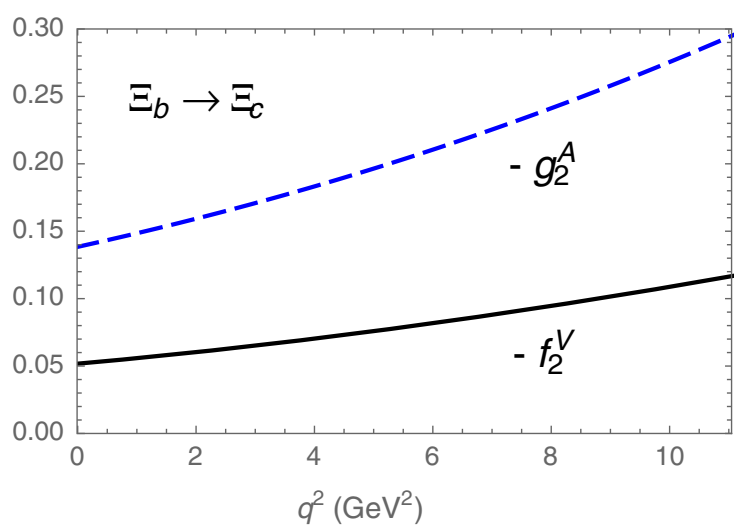

(f)

FIG. 2. Form factors $f_{1,2}\left(q^{2}\right)$ and $g_{1,2}\left(q^{2}\right)$ for $\Lambda_{b} \rightarrow \Lambda_{c}, \Lambda_{c}(2765)$ and $\Xi_{b} \rightarrow \Xi_{c}$ transitions. The transitions are $\mathcal{B}_{b}\left(\overline{\mathbf{3}}_{\mathbf{f}}, 1 / 2^{+}\right) \rightarrow$ $\mathcal{B}_{c}\left(\overline{\mathbf{3}}_{\mathbf{f}}, 1 / 2^{+}\right)$transitions [type (i)].

$$
\begin{aligned}
& \mathcal{A}\left(\mathcal{B}_{b} \rightarrow \mathcal{B}_{c} M^{-}\right) \\
& \quad=\frac{G_{F}}{\sqrt{2}} V_{c b} V_{i j}^{*} a_{1}\left\langle\mathcal{B}_{c}\left|V_{\mu}-A_{\mu}\right| \mathcal{B}_{b}\right\rangle\left\langle M^{-}\left(\bar{q}_{i} q_{j}\right)\left|V^{\mu}-A^{\mu}\right| 0\right\rangle,
\end{aligned}
$$

where $V_{c b, i j}$ are the Cabibbo-Kobayashi-Maskawa (CKM) matrix elements and $a_{1}$ is the effective color-allowed
Wilson coefficient. In naïve factorization $a_{1}$ is given by $c_{1}+c_{2} / N_{c}$ with $c_{1}=1.081$ and $c_{2}=-0.190$ at the scale of $\mu=4.2 \mathrm{GeV}$ [45]. The matrix element $\left\langle\mathcal{B}_{c}\left|V_{\mu}-A_{\mu}\right| \mathcal{B}_{b}\right\rangle$ is given by Eqs. (35) and (36), while $\left\langle M^{-}\left(\bar{q}_{i} q_{j}\right)\left|V^{\mu}-A^{\mu}\right| 0\right\rangle$ for $M=P, V, A$ (with $P, V$ and $A$ stand for pseudoscalar, vector, and axial vector mesons, respectively) are given by 
TABLE IX. The transition form factors for various $\mathcal{B}_{b}\left(\mathbf{6}_{\mathbf{f}}, 1 / 2^{+}\right) \rightarrow \mathcal{B}_{c}\left(\mathbf{6}_{\mathbf{f}}, 1 / 2^{+}\right)$transitions [type (ii)]. We use a three parameter form for these form factors, see Eq. (56).

\begin{tabular}{lcccccccc}
\hline \hline $\mathcal{B}_{b} \rightarrow \mathcal{B}_{c}$ & $F$ & $F(0)$ & $a$ & $b$ & $F$ & $F(0)$ & $a$ & $b$ \\
\hline$\Omega_{b} \rightarrow \Omega_{c}$ & $f_{1}^{V}$ & 0.32 & 0.35 & 1.36 & $g_{1}^{A}-0.11$ & 1.76 & -0.07 \\
& $f_{2}^{V}$ & 0.43 & 1.30 & 2.14 & $g_{2}^{A}-0.013$ & -5.91 & 10.55 \\
$\Omega_{b} \rightarrow \Omega_{c}(3090)$ & $f_{1}^{V}$ & 0.20 & 0.58 & 2.79 & $g_{1}^{A}-0.07$ & 2.47 & 1.27 \\
& $f_{2}^{V}$ & 0.29 & 1.55 & 5.06 & $g_{2}^{A}-0.018-6.18$ & 15.50 \\
\hline \hline
\end{tabular}

$$
\left\langle P\left|V^{\mu}-A^{\mu}\right| 0\right\rangle=i q^{\mu} f_{P}, \quad\left\langle V\left|V^{\mu}-A^{\mu}\right| 0\right\rangle=m_{V} f_{V} \varepsilon_{V}^{*},
$$$$
\left\langle A\left|V^{\mu}-A^{\mu}\right| 0\right\rangle=-m_{A} f_{A} \varepsilon_{A}^{*},
$$

where $f_{P, V, A}$ are the corresponding decay constants.

In type (i) and (ii) transitions $\left[\mathcal{B}_{b}\left(\overline{\mathbf{3}}_{\mathbf{f}}, 1 / 2^{+}\right) \rightarrow\right.$ $\mathcal{B}_{c}\left(\overline{\mathbf{3}}_{\mathbf{f}}, 1 / 2^{+}\right)$and $\mathcal{B}_{b}\left(\mathbf{6}_{\mathbf{f}}, 1 / 2^{+}\right) \rightarrow \mathcal{B}_{c}\left(\mathbf{6}_{\mathbf{f}}, 1 / 2^{+}\right)$transitions], the decay amplitudes are given by [17]

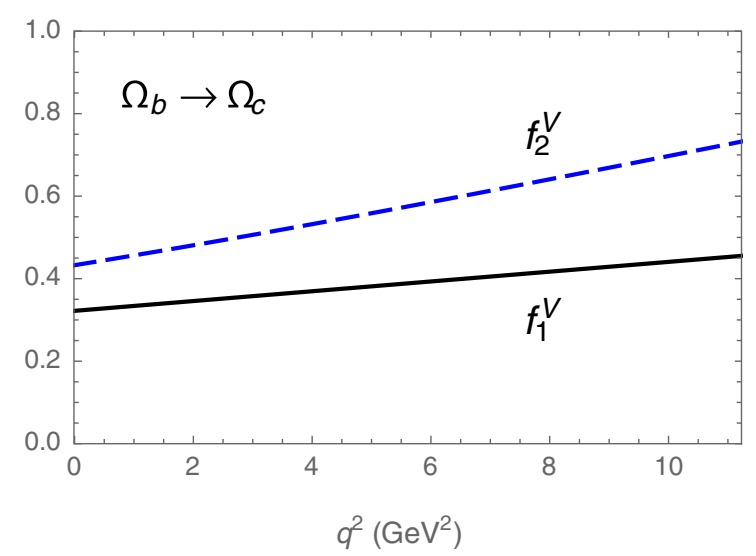

(a)

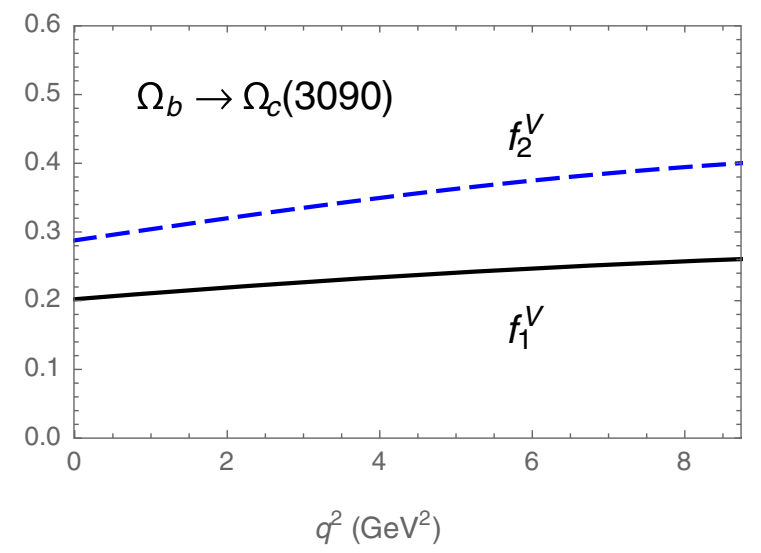

(c)
TABLE $X$. The transition form factors for various $\mathcal{B}_{b}\left(\mathbf{3}_{\mathbf{f}}, 1 / 2^{+}\right) \rightarrow \mathcal{B}_{c}\left(\mathbf{3}_{\mathbf{f}}, 1 / 2^{-}\right)$transitions [type (iii)]. We use a three parameter form for these form factors, see Eq. (56).

\begin{tabular}{lcrrccrcr}
\hline \hline $\mathcal{B}_{b} \rightarrow \mathcal{B}_{c}$ & $F$ & $F(0)$ & $a$ & $b$ & $F$ & $F(0)$ & $a$ & $b$ \\
\hline$\Lambda_{b} \rightarrow \Lambda_{c}(2595)$ & $f_{1}^{A}$ & 0.31 & -0.94 & 1.08 & $g_{1}^{V}$ & 0.27 & 0.40 & -0.04 \\
& $f_{2}^{A}$ & -0.34 & 0.46 & 0.43 & $g_{2}^{V}$ & -0.27 & 0.79 & 0.56 \\
$\Lambda_{b} \rightarrow \Lambda_{c}(2940)$ & $f_{1}^{A}$ & 0.29 & -1.14 & 1.36 & $g_{1}^{V}$ & 0.25 & 0.69 & -0.04 \\
& $f_{2}^{A}$ & -0.33 & 0.41 & 0.58 & $g_{2}^{V}$ & -0.25 & 0.79 & 0.77 \\
$\Xi_{b} \rightarrow \Xi_{c}(2790)$ & $f_{1}^{A}$ & 0.30 & -0.55 & 1.19 & $g_{1}^{V}$ & 0.26 & 1.09 & 0.30 \\
& $f_{2}^{A}-0.37$ & 1.06 & 1.03 & $g_{2}^{V}$ & -0.28 & 1.41 & 1.51 \\
\hline \hline
\end{tabular}

$\mathcal{A}\left(\mathcal{B}_{b} \rightarrow \mathcal{B}_{c} P\right)=i \bar{u}^{\prime}\left(A+B \gamma_{5}\right) u$,

$\mathcal{A}\left(\mathcal{B}_{b} \rightarrow \mathcal{B}_{c} V\right)=\bar{u}^{\prime} \varepsilon^{* \mu}\left(A_{1} \gamma_{\mu} \gamma_{5}+A_{2} P_{\mu}^{\prime} \gamma_{5}+B_{1} \gamma_{\mu}+B_{2} P_{\mu}^{\prime}\right) u$, $\mathcal{A}\left(\mathcal{B}_{b} \rightarrow \mathcal{B}_{c} A\right)=\bar{u}^{\prime} \varepsilon^{* \mu}\left(A_{1}^{\prime} \gamma_{\mu} \gamma_{5}+A_{2}^{\prime} P_{\mu}^{\prime} \gamma_{5}+B_{1}^{\prime} \gamma_{\mu}+B_{2}^{\prime} P_{\mu}^{\prime}\right) u$,

with

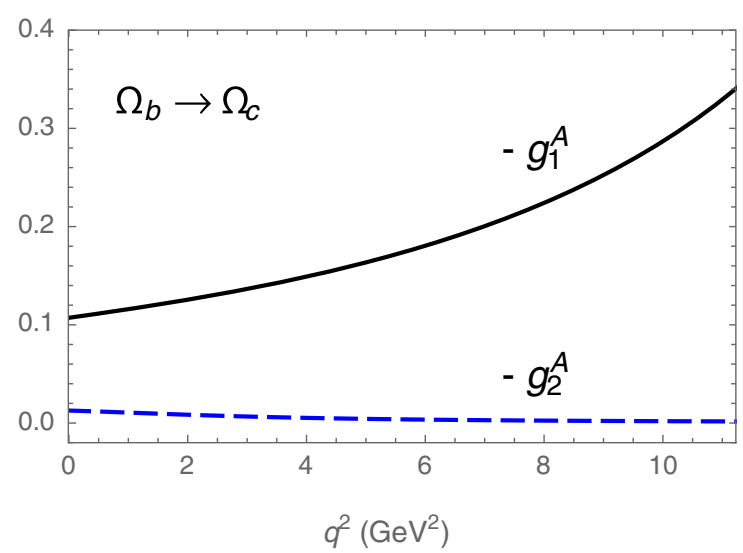

(b)

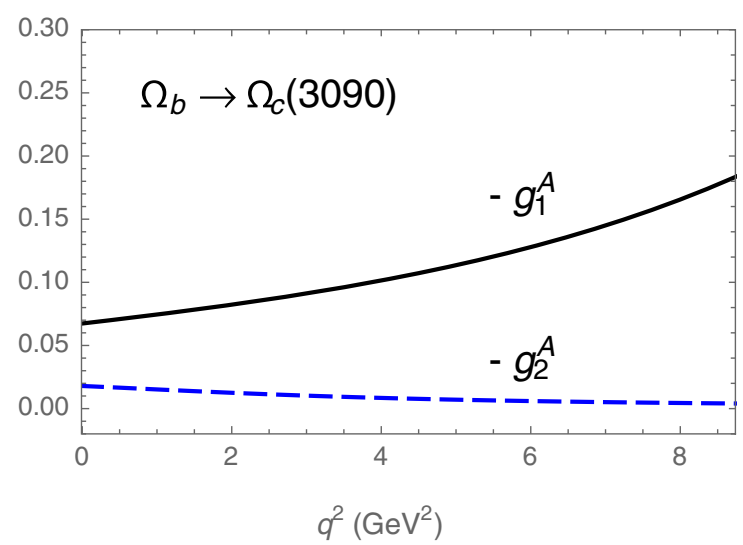

(d)

FIG. 3. Form factors $f_{1,2}\left(q^{2}\right)$ and $g_{1,2}\left(q^{2}\right)$ for $\Omega_{b} \rightarrow \Omega_{c}$ and $\Omega_{c}(3090)$ transitions. The transitions are $\mathcal{B}_{b}\left(\mathbf{6}_{\mathbf{f}}, 1 / 2^{+}\right) \rightarrow \mathcal{B}_{c}\left(\mathbf{6}_{\mathbf{f}}, 1 / 2^{+}\right)$ transitions [type (ii)]. 


$$
\begin{aligned}
A= & \frac{G_{f}}{\sqrt{2}} V_{c b} V_{q_{1} q_{2}}^{*} a_{1} \\
& \times f_{P}\left(\left(M-M^{\prime}\right) f_{1}^{V}\left(m_{P}^{2}\right)+\frac{m_{P}^{2}}{M+M^{\prime}} f_{3}^{V}\left(m_{P}^{2}\right)\right), \\
B= & \frac{G_{f}}{\sqrt{2}} V_{c b} V_{q_{1} q_{2}}^{*} a_{1} f_{P}\left(\left(M+M^{\prime}\right) g_{1}^{A}\left(m_{P}^{2}\right)\right. \\
& \left.+\frac{m_{P}^{2}}{M+M^{\prime}} g_{3}^{A}\left(m_{P}^{2}\right)\right), \\
A_{1}= & -\frac{G_{f}}{\sqrt{2}} V_{c b} V_{q_{1} q_{2}}^{*} a_{1} f_{V} m_{V}\left[g_{1}^{A}\left(m_{V}^{2}\right)+g_{2}^{A}\left(m_{V}^{2}\right) \frac{M-M^{\prime}}{M+M^{\prime}}\right], \\
A_{2}= & -2 \frac{G_{f}}{\sqrt{2}} V_{c b} V_{q_{1} q_{2}}^{*} a_{1} f_{V} m_{V} \frac{g_{2}^{A}\left(m_{V}^{2}\right)}{M+M^{\prime}}, \\
B_{1}= & \frac{G_{f}}{\sqrt{2}} V_{c b} V_{q_{1} q_{2}}^{*} a_{1} f_{V} m_{V}\left[f_{1}^{V}\left(m_{V}^{2}\right)-f_{2}^{V}\left(m_{V}^{2}\right)\right], \\
B_{2}= & 2 \frac{G_{f}}{\sqrt{2}} V_{c b} V_{q_{1} q_{2}}^{*} a_{1} f_{V} m_{V} \frac{f_{2}^{V}\left(m_{V}^{2}\right)}{M+M^{\prime}}, \\
A_{1}^{\prime}= & \frac{G_{f}}{\sqrt{2}} V_{c b} V_{q_{1} q_{2}}^{*} a_{1} f_{A} m_{A}\left[g_{1}^{A}\left(m_{A}^{2}\right)+g_{2}^{A}\left(m_{A}^{2}\right) \frac{M-M^{\prime}}{M+M^{\prime}}\right], \\
A_{2}^{\prime}= & 2 \frac{G_{f}}{\sqrt{2}} V_{c b} V_{q_{1} q_{2}}^{*} a_{1} f_{A} m_{A} \frac{g_{2}^{A}\left(m_{V}^{2}\right)}{M+M^{\prime}}, \\
B_{1}^{\prime}= & -\frac{G_{f}}{\sqrt{2}} V_{c b} V_{q_{1} q_{2}}^{*} a_{1} f_{A} m_{A}\left[f_{1}^{V}\left(m_{A}^{2}\right)-f_{2}^{V}\left(m_{A}^{2}\right)\right], \\
B_{2}^{\prime}= & -2 \frac{G_{f}}{\sqrt{2}} V_{c b} V_{q_{1} q_{2}}^{*} a_{1} f_{A} m_{A} \frac{f_{2}^{V}\left(m_{A}^{2}\right)}{M+M^{\prime}} . \\
& (60) \\
&
\end{aligned}
$$

For the type (iii) transition $\left[\mathcal{B}_{b}\left(\overline{\mathbf{3}}_{\mathbf{f}}, 1 / 2^{+}\right) \rightarrow\right.$ $\mathcal{B}_{c}\left(\overline{\mathbf{3}}_{\mathbf{f}}, 1 / 2^{-}\right)$transition], one simply replaces $f_{i}^{V}$ and $g_{i}^{A}$ in the above equations by $-f_{i}^{A}$ and $-g_{i}^{V}$, respectively.

The decay rates and asymmetries read $[17,46]$

$$
\begin{aligned}
\Gamma\left(\mathcal{B}_{b} \rightarrow \mathcal{B}_{c} P\right)= & \frac{p_{c}}{8 \pi}\left[\frac{\left(M+M^{\prime}\right)^{2}-m_{P}^{2}}{M^{2}}|A|^{2}\right. \\
& \left.+\frac{\left(M-M^{\prime}\right)^{2}-m_{P}^{2}}{M^{2}}|B|^{2}\right], \\
\Gamma\left[\mathcal{B}_{b} \rightarrow \mathcal{B}_{c} V(A)\right]= & \frac{p_{c}}{4 \pi} \frac{E^{\prime}+M^{\prime}}{M}\left[2\left(\left|S^{(\prime)}\right|^{2}+\left|P_{2}^{(\prime)}\right|^{2}\right)\right. \\
& \left.+\frac{E_{V(A)}^{2}}{m_{V(A)}^{2}}\left(\left|S^{(\prime)}+D^{(\prime)}\right|^{2}+\left|P_{1}^{(\prime)}\right|^{2}\right)\right], \quad(61) \\
\alpha\left(\mathcal{B}_{b} \rightarrow \mathcal{B}_{c} P\right)=- & \frac{2 \kappa \operatorname{Re}\left(A^{*} B\right)}{|A|^{2}+\kappa^{2}|B|^{2}}, \\
\alpha\left[\mathcal{B}_{b} \rightarrow \mathcal{B}_{c} V(A)\right] & \frac{4 m_{V(A)}^{2} \operatorname{Re}\left(S^{(\prime) *} P_{2}\right)+2 E_{V}^{2} \operatorname{Re}\left(S^{(\prime)}+D^{(\prime)}\right)^{*} P_{1}^{(\prime)}}{2 m_{V(A)}^{2}\left(\left|S^{(\prime)}\right|^{2}+\left|P_{2}^{(\prime)}\right|^{2}\right)+E_{V(A)}^{2}\left(\left|S^{(\prime)}+D^{(\prime)}\right|^{2}+\left|P_{1}^{(\prime)}\right|^{2}\right)},
\end{aligned}
$$

with $\kappa \equiv p_{c} /\left(E^{\prime}+M^{\prime}\right)$,

$$
\begin{aligned}
S^{(\prime)} & =-A_{1}^{(\prime)}, \quad P_{1}^{(\prime)}=-\frac{p_{c}}{E_{V(A)}}\left(\frac{M+M^{\prime}}{E^{\prime}+M^{\prime}} B_{1}^{(\prime)}+M B_{2}^{(\prime)}\right), \\
P_{2}^{(\prime)} & =\frac{p_{c}}{E^{\prime}+M^{\prime}} B_{1}^{(\prime)}, \\
D^{(\prime)} & =-\frac{p_{c}^{2}}{E_{V(A)}\left(E^{\prime}+M^{\prime}\right)}\left(A_{1}^{(\prime)}-M A_{2}^{(\prime)}\right),
\end{aligned}
$$

where $p_{c}$ is the momentum in the center of mass frame.

All hadron masses and life-times are taken from PDG [1]. The CKM matrix elements are taken from the latest results of the CKM fitter group [47]. The values of decay constants of pseudoscalars are $f_{\pi}=130.2 \mathrm{MeV}, f_{K}=$ $155.6 \mathrm{MeV}, f_{D}=211.9 \mathrm{MeV}$ and $f_{D_{s}}=249.0 \mathrm{MeV}$, which are the center values of the averaged values given in the review by Rosner, Stone and Van de Water in Ref. [1], while those of vectors and the axial-vector particles are $f_{\rho}=216 \mathrm{MeV}, f_{K^{*}}=210 \mathrm{MeV}, f_{D^{*}}=$ $220 \mathrm{MeV}$ and $f_{D_{s}^{*}}=230 \mathrm{MeV}$ and $f_{K^{*}}=-203 \mathrm{MeV}$, which are taken from Ref. [34]. In this work the decay rates are estimated using the naïve factorization approach. Note that in Ref. [48] using QCD factorization the authors obtained $\left|a_{1}(\bar{B} \rightarrow D P)\right|=1.055_{-0.017}^{+0.019}-\left(0.013_{-0.006}^{+0.011}\right) \alpha_{1}^{P}$ with $\alpha_{1}^{\pi}=0$ and $\left|\alpha_{1}^{K}\right|<1$ (see Eq. (230) in [48]). The $\left|a_{1}(D P)\right|$ agrees with the naïve factorization value (Ref. [48] used $a_{1}^{\mathrm{LO}}=1.025$ ) within few $\%$. For estimations, we assign $10 \%$ uncertainty in the effective Wilson coefficient $a_{1}$ and $10 \%$ uncertainty in form factors. Note that in $\mathcal{B}_{b} \rightarrow \mathcal{B}_{c} P$ decays, in principle one needs $f_{3}$ and $g_{3}$ contributions, see Eq. (60). Since these contributions are suppressed by a $m_{P}^{2} /\left(M+M^{\prime}\right)^{2}$ factor compared to the $f_{1}$ and $g_{1}$ terms and $f_{3}, g_{3}$ are expected to be vanishing in the heavy quark limit [25], we shall neglect them, but enlarge the form factor uncertainties to $15 \%$ in $\mathcal{B}_{b} \rightarrow \mathcal{B}_{c} D$ and $\mathcal{B}_{b} \rightarrow \mathcal{B}_{c} D_{s}$ decays.

Note that as shown in Refs. [18,19] nonfactorizable contributions to $\mathcal{B}_{b} \rightarrow \mathcal{B}_{c} P$ nonleptonic decay amplitudes can contribute as large as $30 \%$ comparing to the factorized ones. A precise estimation of nonfactorization contributions is beyond the scope of the present work. ${ }^{5}$ If needed, one can scale up the uncertainties of our numerical results on rates.

The branching ratios for $\mathcal{B}_{b} \rightarrow \mathcal{B}_{c} P, \mathcal{B}_{c} V$ and $\mathcal{B}_{c} A$ decays, with $P=\pi, K, D, D_{s}$, and are summarized in Tables XI and XII. As shown in Table XI the $\Lambda_{b} \rightarrow \Lambda_{c} P$ rates can reasonably reproduce the data within errors. We see that the $\Lambda_{b} \rightarrow \Lambda_{c} \pi$ and $\Lambda_{b} \rightarrow \Lambda_{c} K$ rates prefer lower values, while the $\Lambda_{b} \rightarrow \Lambda_{c} D$ and $\Lambda_{b} \rightarrow \Lambda_{c} D_{s}$ rates prefer higher values. Branching ratios for other modes are

\footnotetext{
${ }^{5}$ One is referred to [28] for a recent attempt on applying QCD factorization to $\Lambda_{b}$ decays.
} 


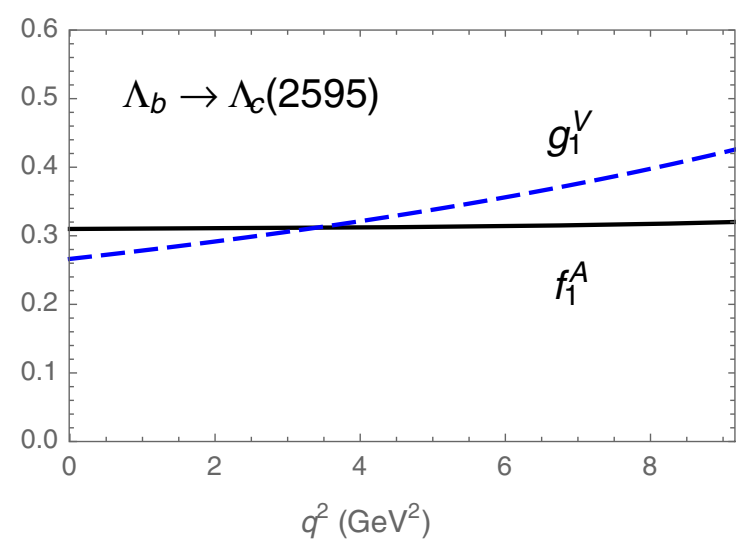

(a)

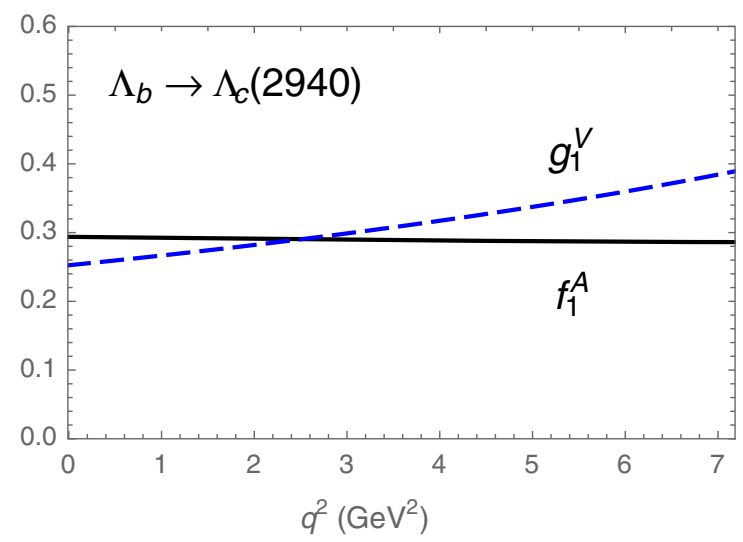

(c)

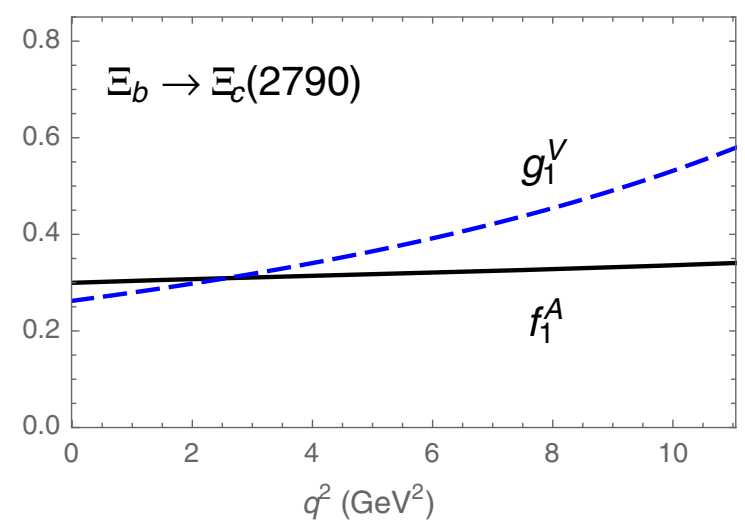

(e)

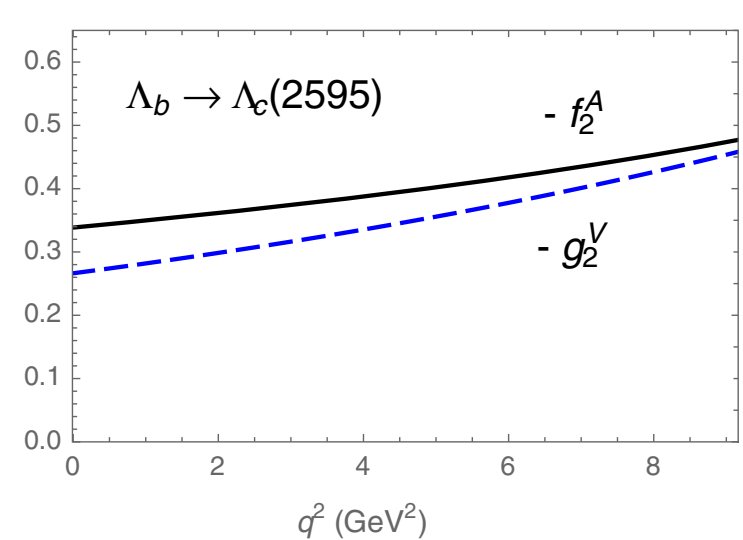

(b)

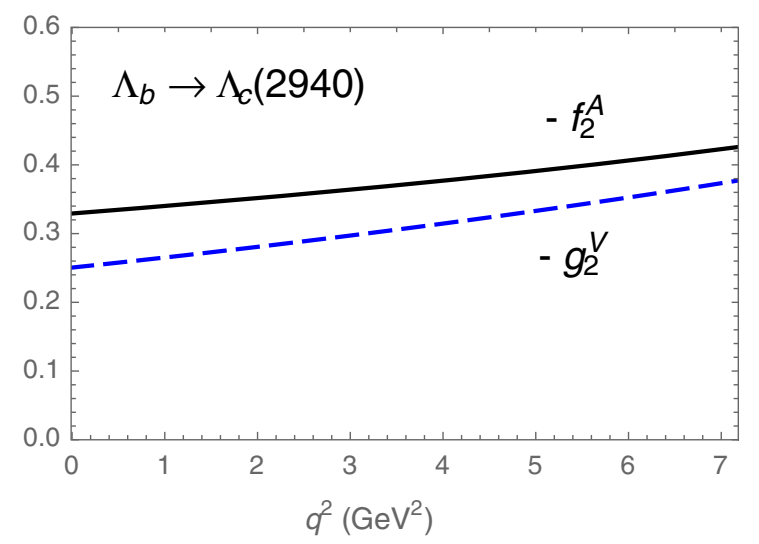

(d)

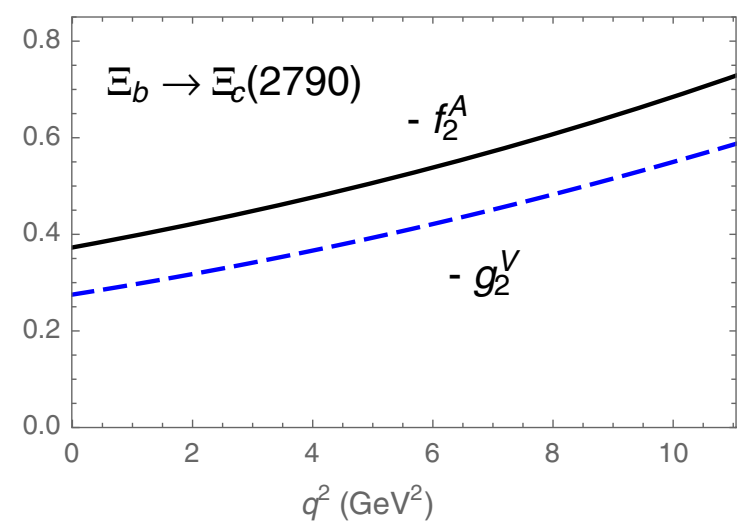

(f)

FIG. 4. Form factors $f_{1,2}\left(q^{2}\right)$ and $g_{1,2}\left(q^{2}\right)$ for $\Lambda_{b} \rightarrow \Lambda_{c}(2595), \Lambda_{c}(2940)$, and $\Xi_{b} \rightarrow \Xi_{c}(2790)$ transitions. The transitions are $\mathcal{B}_{b}\left(\overline{\mathbf{3}}_{\mathbf{f}}, 1 / 2^{+}\right) \rightarrow \mathcal{B}_{c}\left(\overline{\mathbf{3}}_{\mathbf{f}}, 1 / 2^{-}\right)$transitions [type (iii)].

predictions. We find that for $\Lambda_{b}$ decays, we have the following pattern in the decay rates:

$$
\begin{aligned}
\operatorname{Br}\left(\Lambda_{b} \rightarrow \Lambda_{c} P\right) & >\operatorname{Br}\left(\Lambda_{b} \rightarrow \Lambda_{c}(2765) P\right) \\
& >\operatorname{Br}\left(\Lambda_{b} \rightarrow \Lambda_{c}(2595) P\right) \\
& >\operatorname{Br}\left(\Lambda_{b} \rightarrow \Lambda_{c}(2940) P\right)
\end{aligned}
$$

The first two decays are of type (i) transitions $\left[\mathcal{B}_{b}\left(\overline{\mathbf{3}}_{\mathbf{f}}, 1 / 2^{+}\right) \rightarrow \mathcal{B}_{c}\left(\overline{\mathbf{3}}_{\mathbf{f}}, 1 / 2^{+}\right)\right.$transitions $]$, while $\Lambda_{c}(2765)$ is a radial excited state, and the last two decays are of type (iii) transitions $\left[\mathcal{B}_{b}\left(\overline{\mathbf{3}}_{\mathbf{f}}, 1 / 2^{+}\right) \rightarrow \mathcal{B}_{c}\left(\overline{\mathbf{3}}_{\mathbf{f}}, 1 / 2^{-}\right)\right.$transitions], while $\Lambda_{c}(2940)$ is a radial excited state. We see that rates of type (i) transitions are greater than those of type (iii) transitions, and decay rates involving excited states are smaller within the same type. These are reasonable as the configurations of the final states in excited $s$-wave $\mathcal{B}_{c}\left(\overline{\mathbf{3}}_{\mathbf{f}}, 1 / 2^{+}\right)$ state and low lying or excited $p$-wave $\mathcal{B}_{c}\left(\overline{\mathbf{3}}_{\mathbf{f}}, 1 / 2^{-}\right)$states differ from those in the low lying $s$-wave $\mathcal{B}_{b, c}\left(\overline{\mathbf{3}}_{\mathbf{f}}, 1 / 2^{+}\right)$ 
TABLE XI. Branching ratios of $\mathcal{B}_{b} \rightarrow \mathcal{B}_{c} P$ decays. The branching ratios are given in the unit of $10^{-3}$. The asterisks in the first column indicate that the baryons in the final states are radial excited.

\begin{tabular}{|c|c|c|c|c|c|}
\hline Type & Mode & $P=\pi^{-}$ & $P=K^{-}$ & $P=D^{-}$ & $P=D_{s}^{-}$ \\
\hline (i) & $\operatorname{Br}\left(\Lambda_{b} \rightarrow \Lambda_{c} P\right)$ & $4.19_{-1.44}^{+1.94}$ & $0.32_{-0.11}^{+0.15}$ & $0.53_{-0.22}^{+0.32}$ & $13.58_{-5.63}^{+8.15}$ \\
\hline (i) & $B r^{\text {expt. }}\left(\Lambda_{b} \rightarrow \Lambda_{c} P\right)$ & $4.9 \pm 0.4$ & $0.359 \pm 0.030$ & $0.46 \pm 0.06$ & $11.0 \pm 1.0$ \\
\hline (i) & $\operatorname{Br}\left(\Xi_{b}^{0} \rightarrow \Xi_{c}^{+} P\right)$ & $3.08_{-1.06}^{+1.43}$ & $0.23_{-0.08}^{+0.11}$ & $0.43_{-0.18}^{+0.26}$ & $11.20_{-4.65}^{+6.72}$ \\
\hline (i) & $\operatorname{Br}\left(\Xi_{b}^{-} \rightarrow \Xi_{c}^{0} P\right)$ & $3.27_{-1.12}^{+1.52}$ & $0.25_{-0.09}^{+0.12}$ & $0.46_{-0.19}^{+0.28}$ & $11.90_{-4.94}^{+7.14}$ \\
\hline (i)* & $\operatorname{Br}\left[\Lambda_{b} \rightarrow \Lambda_{c}(2765) P\right]$ & $1.58_{-0.54}^{+0.73}$ & $0.12_{-0.04}^{+0.06}$ & $0.18_{-0.07}^{+0.11}$ & $4.39_{-1.82}^{+2.63}$ \\
\hline (ii) & $\operatorname{Br}\left(\Omega_{b} \rightarrow \Omega_{c} P\right)$ & $1.33_{-0.46}^{+0.62}$ & $0.10_{-0.03}^{+0.05}$ & $0.18_{-0.08}^{+0.11}$ & $4.75_{-1.97}^{+2.85}$ \\
\hline (ii) $^{*}$ & $\operatorname{Br}\left[\Omega_{b} \rightarrow \Omega_{c}(3090) P\right]$ & $0.41_{-0.14}^{+0.19}$ & $0.031_{-0.011}^{+0.015}$ & $0.054_{-0.023}^{+0.033}$ & $1.38_{-0.57}^{+0.83}$ \\
\hline (iii) & $\operatorname{Br}\left[\Lambda_{b} \rightarrow \Lambda_{c}(2595) P\right]$ & $1.31_{-0.45}^{+0.61}$ & $0.10_{-0.03}^{+0.05}$ & $0.13_{-0.05}^{+0.08}$ & $3.19_{-1.32}^{+1.92}$ \\
\hline (iii) & $\operatorname{Br}\left[\Xi_{b}^{0} \rightarrow \Xi_{c}^{-}(2790) P\right]$ & $1.27_{-0.44}^{+0.59}$ & $0.10_{-0.03}^{+0.04}$ & $0.14_{-0.06}^{+0.08}$ & $3.41_{-1.41}^{+2.05}$ \\
\hline (iii) & $\operatorname{Br}\left[\Xi_{b}^{-} \rightarrow \Xi_{c}^{0}(2790) P\right]$ & $1.36_{-0.47}^{+0.63}$ & $0.10_{-0.04}^{+0.05}$ & $0.15_{-0.06}^{+0.09}$ & $3.63_{-1.51}^{+2.18}$ \\
\hline (iii)* & $B r\left[\Lambda_{b} \rightarrow \Lambda_{c}(2940) P\right]$ & $0.93_{-0.32}^{+0.43}$ & $0.069_{-0.024}^{+0.032}$ & $0.078_{-0.032}^{+0.047}$ & $1.86_{-0.77}^{+1.12}$ \\
\hline
\end{tabular}

states. Larger mismatch between initial and final state configurations, usually lead to smaller form factors, and, consequently, smaller rates.

The $\Xi_{b} \rightarrow \Xi_{c} P$ modes are of type (i) decays, while $\Xi_{b} \rightarrow$ $\Xi_{c}(2790) P$ decays are of type (iii) decays, where $\Xi_{c}(2790)$ is a $p$-wave baryon. From Table XI we have

$$
\operatorname{Br}\left(\Xi_{b} \rightarrow \Xi_{c} P\right)>\operatorname{Br}\left(\Xi_{b} \rightarrow \Xi_{c}(2790) P\right)
$$

We see again that rates of type (i) transitions are greater than those of type (iii) transitions. Note that the $\Xi_{b} \rightarrow \Xi_{c} P$ rate is slightly smaller than the $\Lambda_{b} \rightarrow \Lambda_{c} P$ rate.

For $\Omega_{b}$ decays, we have

$$
\operatorname{Br}\left(\Omega_{b} \rightarrow \Omega_{c} P\right)>\operatorname{Br}\left(\Omega_{b} \rightarrow \Omega_{c}(3090) P\right) .
$$

These decays are type (ii) decays $\left[\mathcal{B}_{b}\left(\mathbf{6}_{\mathbf{f}}, 1 / 2^{+}\right) \rightarrow\right.$ $\mathcal{B}_{c}\left(\mathbf{6}_{\mathbf{f}}, 1 / 2^{+}\right)$transitions $]$and $\Omega_{c}(3090)$ is a radial excited state. Again the decay rate involving an excite state is smaller. Note that in $\mathcal{B}_{b} \rightarrow \mathcal{B}_{c} P$ decays, rates in type (ii) transition are smaller than those in type (i) transition, but similar to those in type (iii) transition.

The branching ratios for the weak decays $\mathcal{B}_{b} \rightarrow \mathcal{B}_{c} V(A)$, with $V=\rho^{-}, K^{*-}, D^{*-}, D_{s}^{*-}$, and $A=a_{1}^{-}$are summarized in Table XII. We find that for $\Lambda_{b}$ decays, except for $V=\rho^{-}$, we have the following pattern in the decay rates:

$$
\begin{aligned}
\operatorname{Br}\left(\Lambda_{b} \rightarrow \Lambda_{c} V(A)\right) & >\operatorname{Br}\left(\Lambda_{b} \rightarrow \Lambda_{c}(2765) V(A)\right) \\
& \gtrsim \operatorname{Br}\left(\Lambda_{b} \rightarrow \Lambda_{c}(2595) V(A)\right) \\
& >\operatorname{Br}\left(\Lambda_{b} \rightarrow \Lambda_{c}(2940) V(A)\right) .
\end{aligned}
$$

For the case of $V=\rho^{-}$, we have $\operatorname{Br}\left(\Lambda_{b} \rightarrow \Lambda_{c}(2595) \rho\right) \gtrsim$

\begin{tabular}{|c|c|c|c|c|c|c|}
\hline Type & Mode & $M=\rho^{-}$ & $M=K^{*-}$ & $M=D^{*-}$ & $M=D_{s}^{*-}$ & $M=a_{1}^{-}$ \\
\hline (i) & $\operatorname{Br}\left(\Lambda_{b} \rightarrow \Lambda_{c} M\right)$ & $12.39_{-4.28}^{+5.79}$ & $0.63_{-0.22}^{+0.30}$ & $0.79_{-0.28}^{+0.38}$ & $16.33_{-5.81}^{+7.94}$ & $11.53_{-4.01}^{+5.44}$ \\
\hline (i) & $\operatorname{Br}\left(\Xi_{b}^{0} \rightarrow \Xi_{c}^{+} M\right)$ & $9.28_{-3.21}^{+4.34}$ & $0.47_{-0.16}^{+0.22}$ & $0.65_{-0.23}^{+0.32}$ & $13.50_{-4.83}^{+6.62}$ & $8.84_{-3.09}^{+4.19}$ \\
\hline (i) & $\operatorname{Br}\left(\Xi_{b}^{-} \rightarrow \Xi_{c}^{0} M\right)$ & $9.86_{-3.41}^{+4.61}$ & $0.50_{-0.17}^{+0.24}$ & $0.69_{-0.24}^{+0.33}$ & $14.34_{-5.13}^{+7.03}$ & $9.39_{-3.28}^{+4.45}$ \\
\hline (i)* & $\operatorname{Br}\left[\Lambda_{b} \rightarrow \Lambda_{c}(2765) M\right]$ & $4.75_{-1.65}^{+2.22}$ & $0.24_{-0.08}^{+0.11}$ & $0.28_{-0.10}^{+0.14}$ & $5.77_{-2.07}^{+2.83}$ & $4.41_{-1.54}^{+2.09}$ \\
\hline (ii) & $\operatorname{Br}\left(\Omega_{b} \rightarrow \Omega_{c} M\right)$ & $1.63_{-0.75}^{+1.17}$ & $0.082_{-0.037}^{+0.058}$ & $0.088_{-0.035}^{+0.055}$ & $1.84_{-0.71}^{+1.11}$ & $1.41_{-0.63}^{+0.99}$ \\
\hline$(\mathrm{ii})^{*}$ & $\operatorname{Br}\left[\Omega_{b} \rightarrow \Omega_{c}(3090) M\right]$ & $0.54_{-0.24}^{+0.37}$ & $0.027_{-0.012}^{+0.018}$ & $0.030_{-0.011}^{+0.017}$ & $0.62_{-0.23}^{+0.34}$ & $0.47_{-0.20}^{+0.31}$ \\
\hline (iii) & $\operatorname{Br}\left[\Lambda_{b} \rightarrow \Lambda_{c}(2595) M\right]$ & $4.95_{-1.72}^{+2.33}$ & $0.25_{-0.09}^{+0.12}$ & $0.22_{-0.08}^{+0.12}$ & $4.38_{-1.66}^{+2.33}$ & $4.23_{-1.50}^{+2.05}$ \\
\hline (iii) & $\operatorname{Br}\left[\Xi_{b}^{0} \rightarrow \Xi_{c}^{+}(2790) M\right]$ & $5.03_{-1.75}^{+2.37}$ & $0.25_{-0.09}^{+0.12}$ & $0.25_{-0.10}^{+0.13}$ & $5.08_{-1.93}^{+2.70}$ & $4.43_{-1.57}^{+2.14}$ \\
\hline (iii) & $\operatorname{Br}\left[\Xi_{b}^{-} \rightarrow \Xi_{c}^{0}(2790) M\right]$ & $5.35_{-1.86}^{+2.52}$ & $0.27_{-0.09}^{+0.13}$ & $0.27_{-0.10}^{+0.14}$ & $5.41_{-2.05}^{+2.88}$ & $4.72_{-1.67}^{+2.28}$ \\
\hline (iii)* & $\operatorname{Br}\left[\Lambda_{b} \rightarrow \Lambda_{c}(2940) M\right]$ & $3.35_{-1.17}^{+1.58}$ & $0.17_{-0.06}^{+0.08}$ & $0.14_{-0.05}^{+0.07}$ & $2.60_{-1.01}^{+1.42}$ & $2.80_{-1.00}^{+1.36}$ \\
\hline
\end{tabular}
$\operatorname{Br}\left(\Lambda_{b} \rightarrow \Lambda_{c}(2765) \rho\right)$ instead. For the $\Xi_{b} \rightarrow \Xi_{c} M$ mode, we have

TABLE XII. The predicted branching ratios of $\mathcal{B}_{b} \rightarrow \mathcal{B}_{c} V$ and $B_{b} \rightarrow \mathcal{B}_{c} A$ decays. The branching ratios are given in unit of $10^{-3}$. The asterisks in the first column indicate that the baryons in the final states are radial excited. 
TABLE XIII. Various theoretical results on the branching ratios of $\Lambda_{b} \rightarrow \Lambda_{c} M, \Xi_{b} \rightarrow \Xi_{c} M$, and $\Omega_{b} \rightarrow \Omega_{c} M$ decays are compared. The branching ratios are given in the unit of $10^{-3}$. These are to be compared to the experimental branching ratios for $\Lambda_{b} \rightarrow \Lambda_{c} \pi^{-}, \Lambda_{c} K^{-}, \Lambda_{c} D^{-}, \Lambda_{c} D_{s}^{-}$decays, which are $4.9 \pm 0.4,0.359 \pm 0.030$, $0.46 \pm 0.06$, and $11.0 \pm 1.0$ in units of $10^{-3}$, respectively. See text for the results in Ref. [17].

\begin{tabular}{|c|c|c|c|c|c|c|c|c|c|}
\hline Mode & This work & [16] & [17] & {$[18,19]$} & [20] & [21] & [22] & [28] & [30] \\
\hline$\Lambda_{b} \rightarrow \Lambda_{c} \pi^{-}$ & $4.19_{-1.44}^{+1.94}$ & $4.6_{-3.1}^{+2.0}$ & 4.6 & 5.62 & 3.91 & $\ldots$ & 1.75 & 4.96 & $\ldots$ \\
\hline$\Lambda_{b} \rightarrow \Lambda_{c} K^{-}$ & $0.32_{-0.11}^{+0.15}$ & $\ldots$ & $\ldots$ & $\ldots$ & $\ldots$ & $\ldots$ & 0.13 & 0.393 & $\ldots$ \\
\hline$\Lambda_{b} \rightarrow \Lambda_{c} D^{-}$ & $0.53_{-0.22}^{+0.32}$ & $\ldots$ & $\ldots$ & $\ldots$ & $\ldots$ & $\ldots$ & 0.30 & 0.522 & $\ldots$ \\
\hline$\Lambda_{b} \rightarrow \Lambda_{c} D_{s}^{-}$ & $13.58_{-5.63}^{+8.15}$ & $23_{-4}^{+3}$ & 13.7 & $\ldots$ & 12.91 & 22.3 & 7.70 & 12.4 & 14.78 \\
\hline$\Lambda_{b} \rightarrow \Lambda_{c} \rho^{-}$ & $12.39_{-4.28}^{+5.79}$ & $6.6_{-4.0}^{+2.4}$ & 12.9 & $\ldots$ & 10.82 & $\ldots$ & 4.91 & 8.65 & $\ldots$ \\
\hline$\Lambda_{b} \rightarrow \Lambda_{c} K^{*-}$ & $0.63_{-0.22}^{+0.30}$ & $\ldots$ & $\ldots$ & $\ldots$ & $\ldots$ & $\ldots$ & 0.27 & 0.441 & $\ldots$ \\
\hline$\Lambda_{b} \rightarrow \Lambda_{c} D^{*-}$ & $0.79_{-0.28}^{+0.38}$ & $\ldots$ & $\ldots$ & $\ldots$ & $\ldots$ & $\ldots$ & 0.49 & 0.520 & $\ldots$ \\
\hline$\Lambda_{b} \rightarrow \Lambda_{c} D_{s}^{*-}$ & $16.33_{-5.81}^{+7.94}$ & $17.3_{-3.0}^{+2.0}$ & 21.8 & $\ldots$ & 19.83 & 32.6 & 14.14 & 10.5 & 25.16 \\
\hline$\Lambda_{b} \rightarrow \Lambda_{c} a_{1}^{-}$ & $11.53_{-4.01}^{+5.44}$ & $\ldots$ & $\ldots$ & $\ldots$ & $\ldots$ & $\ldots$ & 5.32 & $\ldots$ & $\ldots$ \\
\hline$\Xi_{b}^{0} \rightarrow \Xi_{c}^{+} \pi^{-}$ & $3.08_{-1.06}^{+1.43}$ & $\cdots$ & 4.9 & 7.08 & $\cdots$ & $\cdots$ & $\ldots$ & $\cdots$ & $\cdots$ \\
\hline$\Xi_{b}^{-} \rightarrow \Xi_{c}^{0} \pi^{-}$ & $3.27_{-1.12}^{+1.52}$ & $\ldots$ & 5.2 & 10.13 & $\ldots$ & $\ldots$ & $\ldots$ & $\ldots$ & $\ldots$ \\
\hline$\Xi_{b}^{0} \rightarrow \Xi_{c}^{-} D^{-}$ & $0.43_{-0.18}^{+0.26}$ & $\cdots$ & $\cdots$ & $\ldots$ & $\ldots$ & $\cdots$ & $\ldots$ & $\cdots$ & 0.45 \\
\hline$\Xi_{b}^{0} \rightarrow \Xi_{c}^{-} D_{s}^{-}$ & $11.20_{-4.65}^{+6.72}$ & $\ldots$ & 14.6 & $\ldots$ & $\ldots$ & $\ldots$ & $\ldots$ & $\cdots$ & $\ldots$ \\
\hline$\Xi_{b}^{0} \rightarrow \Xi_{c}^{-} D^{*-}$ & $0.65_{-0.23}^{+0.32}$ & $\ldots$ & $\ldots$ & $\ldots$ & $\ldots$ & $\ldots$ & $\ldots$ & $\ldots$ & 0.95 \\
\hline$\Xi_{b}^{0} \rightarrow \Xi_{c}^{-} D_{s}^{*-}$ & $13.50_{-4.83}^{+6.62}$ & $\ldots$ & 23.1 & $\ldots$ & $\ldots$ & $\ldots$ & $\ldots$ & $\ldots$ & $\ldots$ \\
\hline$\Omega_{b} \rightarrow \Omega_{c} \pi^{-}$ & $1.33_{-0.46}^{+0.62}$ & $\cdots$ & 4.92 & 5.81 & $\cdots$ & $\cdots$ & $\ldots$ & $\cdots$ & 1.88 \\
\hline$\Omega_{b} \rightarrow \Omega_{c} D_{s}^{-}$ & $4.75_{-1.97}^{+2.85}$ & $\ldots$ & 17.9 & $\ldots$ & $\ldots$ & $\ldots$ & $\ldots$ & $\ldots$ & $\ldots$ \\
\hline$\Omega_{b} \rightarrow \Omega_{c} \rho^{-}$ & $1.63_{-0.75}^{+1.17}$ & $\ldots$ & 12.8 & $\ldots$ & $\ldots$ & $\ldots$ & $\ldots$ & $\ldots$ & 5.43 \\
\hline$\Omega_{b} \rightarrow \Omega_{c} D_{s}^{*-}$ & $1.84_{-0.71}^{+1.11}$ & $\cdots$ & 11.5 & $\cdots$ & $\cdots$ & $\cdots$ & $\cdots$ & $\cdots$ & $\cdots$ \\
\hline
\end{tabular}

$$
\operatorname{Br}\left(\Xi_{b} \rightarrow \Xi_{c} V(A)\right)>\operatorname{Br}\left(\Xi_{b} \rightarrow \Xi_{c}(2790) P\right) .
$$

Finally for $\Omega_{b}$ decays, we have

$$
\operatorname{Br}\left(\Omega_{b} \rightarrow \Omega_{c} V(A)\right)>\operatorname{Br}\left(\Omega_{b} \rightarrow \Omega_{c}(3090) V(A)\right)
$$

These patterns are similar to those in $\mathcal{B}_{b} \rightarrow \mathcal{B}_{c} P$ decays. The above patterns reflect the fact that $\Lambda_{c}(2595)$, $\Xi_{c}(2790)$, and $\Lambda_{c}(2940)$ are $p$-wave states, and $\Lambda_{c}(2765), \Lambda_{c}(2940)$, and $\Omega_{c}(3090)$ are radial excited states. Larger mismatch between initial and final state configurations, usually lead to smaller rates. Note that in

TABLE XIV. The predicted up-down asymmetries of $\mathcal{B}_{b} \rightarrow \mathcal{B}_{c} P$ decays. The asymmetries are given in unit of $\%$.

The asterisks in the first column indicate that the baryons in the final states are radial excited.

\begin{tabular}{lccccc}
\hline \hline Type & Mode & $P=\pi^{-}$ & $P=K^{-}$ & $P=D^{-}$ & $P=D_{s}^{-}$ \\
\hline (i) & $\alpha\left(\Lambda_{b} \rightarrow \Lambda_{c} P\right)$ & $-99.99_{-0.00}^{+2.24}$ & $-99.98_{-0.00}^{+2.41}$ & $-98.47_{-1.52}^{+8.91}$ & $-98.06_{-1.87}^{+9.41}$ \\
(i) & $\alpha\left(\Xi_{b}^{0} \rightarrow \Xi_{c}^{+} P\right)$ & $-99.99_{-0.00}^{+2.24}$ & $-99.97_{-0.00}^{+2.41}$ & $-98.40_{-1.59}^{+9.01}$ & $-97.96_{-1.96}^{+9.52}$ \\
(i) & $\alpha\left(\Xi_{b}^{-} \rightarrow \Xi_{c}^{0} P\right)$ & $-99.99_{-0.00}^{+2.24}$ & $-99.97_{-0.00}^{+2.41}$ & $-98.39_{-1.59}^{+9.01}$ & $-97.96_{-1.96}^{+9.53}$ \\
(i)* & $\alpha\left[\Lambda_{b} \rightarrow \Lambda_{c}(2765) P\right]$ & $-100.00_{-0.00}^{+2.14}$ & $-99.98_{-0.00}^{+2.39}$ & $-96.61_{-3.32}^{+10.76}$ & $-95.54_{-4.46}^{+11.49}$ \\
(ii) & $\alpha\left(\Omega_{b} \rightarrow \Omega_{c} P\right)$ & $59.92_{-9.22}^{+9.88}$ & $59.93_{-9.22}^{+9.88}$ & $59.95_{-13.54}^{+14.95}$ & $59.90_{-13.53}^{+14.95}$ \\
(ii)* & $\alpha\left[\Omega_{b} \rightarrow \Omega_{c}(3090) P\right]$ & $60.02_{-9.23}^{+9.88}$ & $60.02_{-9.23}^{+9.88}$ & $59.49_{-13.47}^{+14.93}$ & $59.23_{-13.43}^{+14.92}$ \\
(iii) & $\alpha\left[\Lambda_{b} \rightarrow \Lambda_{c}(2595) P\right]$ & $-98.86_{-1.04}^{+4.77}$ & $-98.84_{-1.05}^{+4.79}$ & $-97.86_{-2.03}^{+9.63}$ & $-97.57_{-2.25}^{+9.93}$ \\
(iii) & $\alpha\left[\Xi_{b}^{0} \rightarrow \Xi_{c}^{+}(2790) P\right]$ & $-99.13_{-0.84}^{+4.44}$ & $-99.12_{-0.84}^{+4.44}$ & $-98.58_{-1.42}^{+8.77}$ & $-98.39_{-1.59}^{+9.02}$ \\
(iii) & $\alpha\left[\Xi_{b}^{-} \rightarrow \Xi_{c}^{0}(2790) P\right]$ & $-99.13_{-0.84}^{+4.44}$ & $-99.12_{-0.84}^{+4.44}$ & $-98.58_{-1.42}^{+8.76}$ & $-98.39_{-1.59}^{+9.02}$ \\
(iii)* & $\alpha\left[\Lambda_{b} \rightarrow \Lambda_{c}(2940) P\right]$ & $-98.86_{-1.03}^{+4.76}$ & $-98.84_{-1.05}^{+4.78}$ & $-97.04_{-2.81}^{+10.41}$ & $-96.36_{-3.60}^{+10.94}$ \\
\hline \hline
\end{tabular}


$\mathcal{B}_{b} \rightarrow \mathcal{B}_{c} V, \mathcal{B}_{c} A$ decays, rates in type (ii) transition are much smaller than those in type (i) transition and are also smaller than those in type (iii) transition.

In Tables XIII, we compare our results on the branching ratios of $\Lambda_{b} \rightarrow \Lambda_{c} M, \quad \Xi_{b} \rightarrow \Xi_{c} M$, and $\Omega_{b} \rightarrow \Omega_{c} M$ decays to those obtained in other works. Note that in the table the results of Ref. [17] are obtained by using Table II in [17] with $a_{1} \simeq 1$, while for the $\mathcal{B}_{b} \rightarrow \mathcal{B}_{c} V$ rates the numerics are corrected by a factor of two, see footnote 7 in [46]. Overall speaking our results agree reasonably well with most of the results obtained in other works. Note that in $\Omega_{b} \rightarrow \Omega_{c} M^{-}$decays, the predicted rates are in general smaller than those obtained in other works, except that the predicted $\operatorname{Br}\left(\Omega_{b} \rightarrow \Omega_{c} \pi^{-}\right)$is close to the one in Ref. [30].

In Tables XIV and XV, we show the predicted up-down asymmetries. The signs are mostly negative, except for those in the type (ii) transitions $\left[\mathcal{B}_{b}\left(\mathbf{6}_{\mathbf{f}}, 1 / 2^{+}\right) \rightarrow\right.$ $\mathcal{B}_{c}\left(\mathbf{6}_{\mathbf{f}}, 1 / 2^{+}\right)$transitions]. These signs can be easily traced to the relative signs of their form factors. Most of these asymmetries are large in sizes. Note that in type (i) and (ii) cases, the asymmetries $\left|\alpha\left(\mathcal{B}_{b} \rightarrow B_{c} D_{(s)}^{*}\right)\right|$ are smaller than $\left|\alpha\left(\mathcal{B}_{b} \rightarrow B_{c} \rho\right)\right|,\left|\alpha\left(\mathcal{B}_{b} \rightarrow B_{c} K^{*}\right)\right|$, and $\left|\alpha\left(\mathcal{B}_{b} \rightarrow B_{c} a^{-}\right)\right|$.

In Tables XVI, we compare our results on the up-down asymmetries of $\Lambda_{b} \rightarrow \Lambda_{c} M, \Xi_{b} \rightarrow \Xi_{c} M$, and $\Omega_{b} \rightarrow \Omega_{c} M$

TABLE XV. The predicted up-down asymmetries of $B_{b} \rightarrow \mathcal{B}_{c} V$ and $B_{b} \rightarrow \mathcal{B}_{c} A$ decays. The asymmetries are given in unit of $\%$. The asterisks in the first column indicate that the baryons in the final states are radial excited.

\begin{tabular}{lcccccc}
\hline \hline Type & Mode & $M=\rho^{-}$ & $M=K^{*-}$ & $M=D^{*-}$ & $M=D_{s}^{*-}$ & $M=a_{1}^{-}$ \\
\hline (i) & $\alpha\left(\Lambda_{b} \rightarrow \Lambda_{c} M\right)$ & $-90.50_{-0.23}^{+2.07}$ & $-87.50_{-0.30}^{+2.34}$ & $-48.19_{-2.75}^{+4.21}$ & $-44.10_{-2.94}^{+4.19}$ & $-77.40_{-0.74}^{+3.15}$ \\
(i) & $\alpha\left(\Xi_{b}^{0} \rightarrow \Xi_{c}^{+} M\right)$ & $-90.86_{-0.27}^{+2.04}$ & $-87.97_{-0.35}^{+2.33}$ & $-49.52_{-2.90}^{+4.41}$ & $-45.45_{-3.12}^{+4.41}$ & $-78.18_{-0.78}^{+3.19}$ \\
(i) & $\alpha\left(\Xi_{b}^{-} \rightarrow \Xi_{c}^{0} M\right)$ & $-90.86_{-0.27}^{+2.04}$ & $-87.97_{-0.35}^{+2.33}$ & $-49.53_{-2.90}^{+4.41}$ & $-45.46_{-3.12}^{+4.41}$ & $-78.18_{-0.78}^{+3.19}$ \\
(i)* & $\alpha\left[\Lambda_{b} \rightarrow \Lambda_{c}(2765) M\right]$ & $-88.29_{-0.26}^{+2.32}$ & $-84.65_{-0.34}^{+2.69}$ & $-38.47_{-3.80}^{+4.54}$ & $-33.83_{-3.87}^{+4.36}$ & $-72.48_{-1.23}^{+3.78}$ \\
(ii) & $\alpha\left(\Omega_{b} \rightarrow \Omega_{c} M\right)$ & $85.23_{-14.93}^{+11.84}$ & $85.99_{-14.89}^{+11.33}$ & $89.18_{-8.69}^{+6.02}$ & $87.80_{-7.38}^{+5.74}$ & $88.34_{-14.43}^{+9.18}$ \\
(ii)* & $\alpha\left[\Omega_{b} \rightarrow \Omega_{c}(3090) M\right]$ & $84.20_{-14.41}^{+11.75}$ & $85.12_{-14.29}^{+11.12}$ & $83.23_{-6.34}^{+5.78}$ & $79.90_{-7.25}^{+6.23}$ & $87.70_{-13.37}^{+8.44}$ \\
(iii) & $\alpha\left[\Lambda_{b} \rightarrow \Lambda_{c}(2595) M\right]$ & $-83.26_{-4.51}^{+7.00}$ & $-80.37_{-4.14}^{+6.59}$ & $-39.26_{-3.26}^{+4.24}$ & $-34.49_{-3.76}^{+4.50}$ & $-70.45_{-2.89}^{+5.21}$ \\
(iii) & $\alpha\left[\Xi_{b}^{0} \rightarrow \Xi_{c}^{+}(2790) M\right]$ & $-83.09_{-4.52}^{+7.00}$ & $-80.16_{-4.13}^{+6.58}$ & $-37.67_{-3.63}^{+4.42}$ & $-32.69_{-4.11}^{+4.64}$ & $-70.02_{-2.86}^{+5.16}$ \\
(iii) & $\alpha\left[\Xi_{b}^{-} \rightarrow \Xi_{c}^{0}(2790) M\right]$ & $-83.10_{-4.52}^{+7.00}$ & $-80.17_{-4.14}^{+6.58}$ & $-37.72_{-3.63}^{+4.42}$ & $-32.74_{-4.11}^{+4.64}$ & $-70.04_{-2.86}^{+5.16}$ \\
(iii)* & $\alpha\left[\Lambda_{b} \rightarrow \Lambda_{c}(2940) M\right]$ & $-82.69_{-3.64}^{+6.30}$ & $-79.33_{-3.10}^{+5.74}$ & $-29.73_{-4.67}^{+4.94}$ & $-24.03_{-4.86}^{+4.80}$ & $-67.60_{-1.88}^{+3.87}$ \\
\hline \hline
\end{tabular}

TABLE XVI. Various theoretical results on the up-down asymmetries $(\alpha)$ of $\Lambda_{b} \rightarrow \Lambda_{c} M, \Xi_{b} \rightarrow \Xi_{c} M$, and $\Omega_{b} \rightarrow$ $\Omega_{c} M$ decays are compared. The asymmetries are given in the unit of $\%$.

\begin{tabular}{lcccccccc}
\hline \hline Mode & This work & {$[16]$} & {$[17]$} & {$[18,19]$} & {$[21]$} & {$[22]$} & {$[28]$} & {$[30]$} \\
\hline$\Lambda_{b} \rightarrow \Lambda_{c} \pi^{-}$ & $-99.99_{-0.00}^{+2.24}$ & -100 & -99 & -99 & $\ldots$ & -99.9 & -99.8 & $\ldots$ \\
$\Lambda_{b} \rightarrow \Lambda_{c} K^{-}$ & $-99.98_{-0.00}^{+2.41}$ & $\ldots$ & $\ldots$ & $\ldots$ & $\ldots$ & -100 & -100 & $\ldots$ \\
$\Lambda_{b} \rightarrow \Lambda_{c} D^{-}$ & $-98.47_{-1.52}^{+8.91}$ & $\ldots$ & $\ldots$ & $\ldots$ & $\ldots$ & -98.7 & -99.9 & -98.9 \\
$\Lambda_{b} \rightarrow \Lambda_{c} D_{s}^{-}$ & $-98.06_{-1.87}^{+9.41}$ & -99.1 & -99 & $\ldots$ & -98 & -98.4 & -100 & -98.6 \\
$\Lambda_{b} \rightarrow \Lambda_{c} \rho^{-}$ & $-90.50_{-0.23}^{+2.07}$ & -90.3 & -88 & $\ldots$ & $\ldots$ & -89.8 & -88.8 & $\ldots$ \\
$\Lambda_{b} \rightarrow \Lambda_{c} K^{*-}$ & $-87.50_{-0.30}^{+2.34}$ & $\ldots$ & $\ldots$ & $\ldots$ & $\ldots$ & -86.5 & -85.9 & $\ldots$ \\
$\Lambda_{b} \rightarrow \Lambda_{c} D^{*-}$ & $-48.19_{-2.75}^{+4.21}$ & $\ldots$ & $\ldots$ & $\ldots$ & $\ldots$ & -45.9 & -47.8 & $\ldots$ \\
$\Lambda_{b} \rightarrow \Lambda_{c} D_{s}^{*-}$ & $-44.10_{-2.19}^{+4.94}$ & -43.7 & -36 & $\ldots$ & -40 & -41.9 & -43.9 & -36.4 \\
$\Lambda_{b} \rightarrow \Lambda_{c} a_{1}^{-}$ & $-77.40_{-0.74}^{+3.15}$ & $\ldots$ & $\ldots$ & $\ldots$ & $\ldots$ & -75.8 & $\ldots$ & $\ldots$ \\
$\Xi_{b}^{0} \rightarrow \Xi_{c}^{+} \pi^{-}$ & $-99.99_{-0.00}^{+2.24}$ & $\ldots$ & -100 & -100 & $\ldots$ & $\ldots$ & $\ldots$ & $\ldots$ \\
$\Xi_{b}^{-} \rightarrow \Xi_{c}^{0} \pi^{-}$ & $-99.99_{-0.00}^{+2.24}$ & $\ldots$ & -100 & -97 & $\ldots$ & $\ldots$ & $\ldots$ & $\ldots$ \\
$\Xi_{b}^{0} \rightarrow \Xi_{c}^{+} D_{s}^{-}$ & $-97.96_{-1.96}^{+9.52}$ & $\ldots$ & -99 & $\ldots$ & $\ldots$ & $\ldots$ & $\ldots$ & $\ldots$ \\
$\Xi_{b}^{0} \rightarrow \Xi_{c}^{-} D_{s}^{*-}$ & $-45.45_{-3.12}^{+4.41}$ & $\ldots$ & -36 & $\ldots$ & $\ldots$ & $\ldots$ & $\ldots$ & $\ldots$ \\
$\Omega_{b} \rightarrow \Omega_{c} \pi^{-}$ & $59.92_{-9.22}^{+9.88}$ & $\ldots$ & 51 & 60 & $\ldots$ & $\ldots$ & $\ldots$ & $\ldots$ \\
$\Omega_{b} \rightarrow \Omega_{c} D_{s}^{-}$ & $59.90_{-13.53}^{+14.95}$ & $\ldots$ & 42 & $\ldots$ & $\ldots$ & $\ldots$ & $\ldots$ & $\ldots$ \\
$\Omega_{b} \rightarrow \Omega_{c} \rho^{-}$ & $85.23_{-14.93}^{+1.84}$ & $\ldots$ & 53 & $\ldots$ & $\ldots$ & $\ldots$ & $\ldots$ & $\ldots$ \\
$\Omega_{b} \rightarrow \Omega_{c} D_{s}^{*-}$ & $87.80_{-7.38}^{+5.74}$ & $\ldots$ & 64 & $\ldots$ & $\ldots$ & $\ldots$ & $\ldots$ & $\ldots$ \\
\hline \hline
\end{tabular}


decays to those obtained in other works. Our results agree well in signs and magnitudes of the asymmetries with those in other works, except in $\Omega_{b} \rightarrow \Omega_{c} D_{s}^{-}, \Omega_{c} \rho^{-}, \Omega_{c} D_{s}^{*-}$ decays the predicted asymmetries are larger than those in ref. [17], but nevertheless the signs agree.

The predictions on rates and asymmetries presented in Tables XI, XII, XIV, and XV can be verified experimentally. These information may shed light on the quantum numbers of $\Lambda_{c}(2765), \Lambda_{c}(2940)$, and $\Omega_{c}(3090)$.

\section{CONCLUSIONS}

We began with a brief overview of the charmed and bottom baryon spectroscopy and discussed their possible structure and $J^{P}$ assignment in the quark model. As a working assumption we follow Ref. [4] to assign the quantum numbers of some singled charmed states. It is known that among low lying singly bottom baryons, only $\Lambda_{b}, \Xi_{b}$ and $\Omega_{b}$ decay weakly. Consequently, we study $\Lambda_{b} \rightarrow \Lambda_{c}^{(*, *)} M^{-}, \quad \Xi_{b} \rightarrow \Xi_{c}^{(* *)} M^{-}, \quad$ and $\quad \Omega_{b} \rightarrow \Omega_{c}^{(*)} M^{-}$ decays with $M=\pi, K, \rho, K^{*}, D, D_{s}, D^{*}, D_{s}^{*}, a_{1}, \Lambda_{c}^{(*, * *)}=$ $\Lambda_{c}, \Lambda_{c}(2595), \Lambda_{c}(2765), \Lambda_{c}(2940), \Xi_{c}^{(* *)}=\Xi_{c}, \Xi_{c}(2790)$, and $\Omega_{c}^{(*)}=\Omega_{c}, \Omega_{c}(3090)$, in this work. There are three types of transitions, namely $\mathcal{B}_{b}\left(\overline{\mathbf{3}}_{\mathbf{f}}, 1 / 2^{+}\right)$to $\mathcal{B}_{c}\left(\overline{\mathbf{3}}_{\mathbf{f}}, 1 / 2^{+}\right)$, $\mathcal{B}_{b}\left(\mathbf{6}_{\mathbf{f}}, 1 / 2^{+}\right)$to $\mathcal{B}_{c}\left(\mathbf{6}_{\mathbf{f}}, 1 / 2^{+}\right)$and $\mathcal{B}_{b}\left(\overline{\mathbf{3}}_{\mathbf{f}}, 1 / 2^{+}\right)$to $\mathcal{B}_{c}\left(\overline{\mathbf{3}}_{\mathbf{f}}, 1 / 2^{-}\right)$transitions. The bottom baryon to charmed baryon form factors are calculated using the light-front quark model. The formulas for the $\mathcal{B}_{b}\left(\mathbf{6}_{\mathbf{f}}, 1 / 2^{+}\right)$to $\mathcal{B}_{c}\left(\mathbf{6}_{\mathbf{f}}, 1 / 2^{+}\right)$and $\mathcal{B}_{b}\left(\overline{\mathbf{3}}_{\mathbf{f}}, 1 / 2^{+}\right)$to $\mathcal{B}_{c}\left(\overline{\mathbf{3}}_{\mathbf{f}}, 1 / 2^{-}\right)$transition form factors are new results. Those with an excited state in the $\mathcal{B}_{b}\left(\overline{\mathbf{3}}_{\mathbf{f}}, 1 / 2^{+}\right)$to $\mathcal{B}_{c}\left(\overline{\mathbf{3}}_{\mathbf{f}}, 1 / 2^{+}\right)$transition are also new.

Numerical results of form factors, decay rates and updown asymmetries in these decays are shown. We see that rates of $\mathcal{B}_{b}\left(\overline{\mathbf{3}}_{\mathbf{f}}, 1 / 2^{+}\right) \rightarrow \mathcal{B}_{c}\left(\overline{\mathbf{3}}_{\mathbf{f}}, 1 / 2^{+}\right)$transitions [type (i)] are greater than those of $\mathcal{B}_{b}\left(\overline{\mathbf{3}}_{\mathbf{f}}, 1 / 2^{+}\right) \rightarrow \mathcal{B}_{c}\left(\overline{\mathbf{3}}_{\mathbf{f}}, 1 / 2^{-}\right)$ [type (iii)] transitions, and decay rates involving excited states are smaller within the same type of transition. These are reasonable as the configurations of the final states in excited $\mathcal{B}_{c}\left(\overline{\mathbf{3}}_{\mathbf{f}}, 1 / 2^{+}\right)$state and low lying or excited $\mathcal{B}_{c}\left(\overline{\mathbf{3}}_{\mathbf{f}}, 1 / 2^{-}\right) p$-wave states differ from those in the low lying $\mathcal{B}_{b, c}\left(\overline{\mathbf{3}}_{\mathbf{f}}, 1 / 2^{+}\right)$states. Larger mismatch between initial and final state configurations, usually lead to smaller form factors, and, consequently, smaller rates. Furthermore, we find that in $\mathcal{B}_{b} \rightarrow \mathcal{B}_{c} P$ decays, rates in $\mathcal{B}_{b}\left(\mathbf{6}_{\mathbf{f}}, 1 / 2^{+}\right) \rightarrow \mathcal{B}_{c}\left(\mathbf{6}_{\mathbf{f}}, 1 / 2^{+}\right)$[type (ii)] transition are smaller than those in $\mathcal{B}_{b}\left(\overline{\mathbf{3}}_{\mathbf{f}}, 1 / 2^{+}\right) \rightarrow \mathcal{B}_{c}\left(\overline{\mathbf{3}}_{\mathbf{f}}, 1 / 2^{+}\right)$[type (i)] transition, but similar to those in $\mathcal{B}_{b}\left(\overline{\mathbf{3}}_{\mathbf{f}}, 1 / 2^{+}\right) \rightarrow$ $\mathcal{B}_{c}\left(\overline{\mathbf{3}}_{\mathbf{f}}, 1 / 2^{-}\right)$[type (iii)] transition, while in $\mathcal{B}_{b} \rightarrow$ $\mathcal{B}_{c} V, \mathcal{B}_{c} A$ decays, rates in $\mathcal{B}_{b}\left(\mathbf{6}_{\mathbf{f}}, 1 / 2^{+}\right) \rightarrow \mathcal{B}_{c}\left(\mathbf{6}_{\mathbf{f}}, 1 / 2^{+}\right)$ [type (ii)] transition are much smaller than those in $\mathcal{B}_{b}\left(\overline{\mathbf{3}}_{\mathbf{f}}, 1 / 2^{+}\right) \rightarrow \mathcal{B}_{c}\left(\overline{\mathbf{3}}_{\mathbf{f}}, 1 / 2^{+}\right)$[type (i)] transition and are also smaller than those in $\mathcal{B}_{b}\left(\overline{\mathbf{3}}_{\mathbf{f}}, 1 / 2^{+}\right) \rightarrow \mathcal{B}_{c}\left(\overline{\mathbf{3}}_{\mathbf{f}}, 1 / 2^{-}\right)$ [type (iii)] transition.
For the up-down asymmetries, the signs are mostly negative, except for those in the $\mathcal{B}_{b}\left(\mathbf{6}_{\mathbf{f}}, 1 / 2^{+}\right) \rightarrow$ $\mathcal{B}_{c}\left(\mathbf{6}_{\mathbf{f}}, 1 / 2^{+}\right)$[type (ii)] transition. These asymmetries are large in sizes. Note that in type (i) and (ii) cases, the asymmetries $\left|\alpha\left(\mathcal{B}_{b} \rightarrow B_{c} D_{(s)}^{*}\right)\right|$ are smaller than $\left|\alpha\left(\mathcal{B}_{b} \rightarrow B_{c} \rho\right)\right|,\left|\alpha\left(\mathcal{B}_{b} \rightarrow B_{c} K^{*}\right)\right|$ and $\left|\alpha\left(\mathcal{B}_{b} \rightarrow B_{c} a^{-}\right)\right|$.

We compare our results of rates and asymmetries of $\Lambda_{b} \rightarrow \Lambda_{c} M, \quad \Xi_{b} \rightarrow \Xi_{c} M$, and $\Omega_{b} \rightarrow \Omega_{c} M$ decays to existing results in other works. In most cases the agreements are reasonably well.

Predictions on rates and asymmetries can be checked experimentally. The study on these decay modes may shed light on the quantum numbers of the charmed baryons, as the decays depend on bottom baryon to charmed baryon form factors, which are sensitive to the configurations of the final state charmed baryons. This work can be further extended by including transitions having spin-3/2 baryons in the final states. The result will be reported elsewhere.

\section{ACKNOWLEDGMENTS}

The author likes to thank Hai-Yang Cheng for discussion. This research was supported in part by the Ministry of Science and Technology of the Republic of China (Taiwan) under Grant No. 106-2112-M-033-004-MY3.

\section{APPENDIX A: VERTEX FUNCTIONS}

\section{Some useful identities}

We collect some useful identities for the derivation of vertex functions in the following parts. Relations involving Melosh transform for spin-1/2 and spin-1 particles are given by

$$
\begin{aligned}
& \left\langle\lambda_{1}\left|\mathcal{R}_{M}^{\dagger}\left(x_{1}, k_{1 \perp}, m_{1}\right)\right| s_{1}\right\rangle \bar{u}_{D}\left(k_{1}, s_{1}\right) \\
& =\bar{u}\left(k_{1}, \lambda_{1}\right) \frac{u_{D}\left(k_{1}, s_{1}\right) \bar{u}_{D}\left(k_{1}, s_{1}\right)}{2 m_{1}}=\bar{u}\left(k_{1}, \lambda_{1}\right), \\
& \left\langle\lambda_{2}\left|\mathcal{R}_{M}^{\dagger}\left(x_{2}, k_{2 \perp}, m_{2}\right)\right| s_{2}\right) \varepsilon_{I}^{*}\left(k_{2}, s_{2}\right) \\
& \quad=-\varepsilon_{L F}^{*}\left(k_{2}, \lambda_{2}\right) \cdot \varepsilon_{I}\left(k_{2}, s_{2}\right) \varepsilon_{I}^{*}\left(k_{2}, s_{2}\right)=\varepsilon_{L F}^{*}\left(k_{2}, \lambda_{2}\right),
\end{aligned}
$$

where the familiar formulas of polarization sums are used, $u_{D}$ and $\varepsilon_{I}$ are the spinor and polarization vector in the instant form, while $u$ and $\varepsilon_{L F}$ are the ones in the light-front form. Note that in the particle rest frame, $\varepsilon_{I}$ and $\varepsilon_{L F}$ are identical, and likewise $u_{D}$ and $u$ are identical.

The relevant Clebsch-Gordan coefficients can be expressed in compact forms: 


$$
\begin{aligned}
\left\langle\frac{1}{2} 0 ; s_{1} 0 \mid \frac{1}{2} 0 ; \frac{1}{2} J_{z}\right\rangle= & \chi_{s_{1}}^{\dagger} \cdot \chi_{J_{z}} \\
= & \frac{1}{\sqrt{\left(M_{0}+m_{1}\right)^{2}-m_{2}^{2}}} \bar{u}_{D}\left(k_{1}, s_{1}\right) \\
& \times u\left(k_{1}+k_{2}, J_{z}\right), \\
\left\langle\frac{1}{2} 1 ; s_{1} s_{2} \mid \frac{1}{2} 1 ; \frac{1}{2} J_{z}\right\rangle= & \frac{1}{\sqrt{3}} \chi_{s_{1}}^{\dagger} \vec{\sigma} \cdot \vec{\varepsilon}_{I}^{*}\left(k_{1}+k_{2}, s_{2}\right) \chi_{J_{z}} \\
= & \frac{1}{\sqrt{3\left[\left(M_{0}+m_{1}\right)^{2}-m_{2}^{2}\right]}} \bar{u}_{D}\left(k_{1}, s_{1}\right) \\
& \times \gamma_{5} \phi_{I}^{*}\left(k_{1}+k_{2}, s_{2}\right) u\left(k_{1}+k_{2}, J_{z}\right) .
\end{aligned}
$$

The following relation of the polarization vectors will be needed,

$$
\begin{aligned}
& \varepsilon_{I}^{\mu}\left(k_{2}, s_{2}\right)=\varepsilon_{I}^{\mu}\left(\bar{P}, s_{2}\right)-\frac{M_{0} k_{2}^{\mu}+m_{2} \bar{P}^{\mu}}{m_{2} M_{0}} \frac{\varepsilon_{I}\left(\bar{P}, s_{2}\right) \cdot k_{2}}{e_{2}+m_{2}}, \\
& \varepsilon_{I \mu}^{*}(\bar{P}, m) \varepsilon_{I \nu}(\bar{P}, m)=-g_{\mu \nu}+\frac{\bar{P}_{\mu} \bar{P}_{\nu}}{M_{0}} .
\end{aligned}
$$

Derivations of some of the above relations will be given briefly in the following discussion.

The relations in Eqs. (A3) and (A4) can be easily proved by using the explicit expression of the Dirac spinors. Explicitly, we use

$$
\begin{aligned}
u_{D}\left(k_{1}, s\right) & =\frac{k_{1} \cdot \gamma+m_{1}}{\sqrt{e_{1}+m_{1}}}\left(\begin{array}{c}
\chi_{s} \\
0
\end{array}\right) \\
& =\frac{1}{\sqrt{e_{1}+m_{1}}}\left(\begin{array}{c}
\left(e_{1}+m_{1}\right) \chi_{s} \\
\vec{\sigma} \cdot \vec{p} \chi_{s}
\end{array}\right), \\
u\left(k_{1}+k_{2}, \lambda\right) & =\frac{\left(k_{1}+k_{2}\right) \cdot \gamma+M_{0}}{\sqrt{2 M_{0}}} \gamma^{+} \gamma^{0}\left(\begin{array}{c}
\chi_{\lambda} \\
0
\end{array}\right) \\
& =\sqrt{2 M_{0}}\left(\begin{array}{c}
\chi_{\lambda} \\
0
\end{array}\right),
\end{aligned}
$$

the standard Dirac representation of $\gamma^{\mu}, \gamma_{5}$, and $\varepsilon_{I}\left(k_{1}+k_{2}, s\right)=(0, \vec{\varepsilon}(s))$ with $\vec{\varepsilon}( \pm 1)=\mp(1, \pm i, 0) / \sqrt{2}$, $\vec{\varepsilon}(0)=(0,0,1)$.

The derivation of Eq. (A5) is a bit tricky. We want to express $\varepsilon_{I}\left(k_{2}, s_{2}\right)$ in terms of $\varepsilon_{I}\left(k_{1}+k_{2}, s_{2}\right)$, which are polarization vectors in the instant form and in the rest frame of $\bar{P}=k_{1}+k_{2}=\left(M_{0}, \overrightarrow{0}\right)$. It is useful to note that $\varepsilon_{I}\left(k_{1}+k_{2}, s_{2}\right)$ and the polarization vector of particle 2 in its rest fame, $\varepsilon_{I}\left(\left(m_{2}, \overrightarrow{0}\right), s_{2}\right)$, are indeed identical, as both are equal to $\left(0, \vec{\varepsilon}\left(s_{2}\right)\right)$ with $\vec{\varepsilon}( \pm 1)=\mp(1, \pm i, 0) / \sqrt{2}$, $\vec{\varepsilon}(0)=(0,0,1)$, i.e.,
$\varepsilon_{I}\left(\bar{P}=\left(M_{0}, \overrightarrow{0}\right), s_{2}\right)=\varepsilon_{I}\left(\left(m_{2}, \overrightarrow{0}\right), s_{2}\right)=\left(0, \vec{\varepsilon}\left(s_{2}\right)\right)$

Therefore, $\varepsilon_{I}\left(k_{2}, s_{2}\right)$ and $\varepsilon_{I}\left(k_{1}+k_{2}, s_{2}\right)$ [or $\varepsilon_{I}\left(\left(m_{2}, \overrightarrow{0}\right), s_{2}\right)$ ] can be related by a suitable Lorentz boost.

When the Lorentz boost, which brings particle 2 with momentum from $\left(m_{2}, \overrightarrow{0}\right)$ to $k_{2}=\left(e_{2}, \vec{k}_{2}\right)$, acts on a generic four vector $A^{\mu}$, we have the following transformations:

$$
A^{0} \rightarrow A^{0} \frac{e_{2}}{m_{2}}+\vec{A} \cdot \frac{\vec{k}_{2}}{m_{2}}, \quad \vec{A} \rightarrow \vec{A}+\frac{\vec{k}_{2}}{m_{2}}\left(\frac{\vec{k}_{2} \cdot \vec{A}}{e_{2}+m_{2}}\right)+A^{0} \frac{\vec{k}_{2}}{m_{2}} .
$$

One can easily check that it indeed brings $\left(m_{2}, \overrightarrow{0}\right)$ to $k_{2}$. Now by boosting the diquark polarization vector, $\varepsilon_{I}\left(\left(m_{2}, \overrightarrow{0}\right), s_{2}\right)=\left(0, \vec{\varepsilon}\left(s_{2}\right)\right)$, from its rest frame to $\varepsilon_{I}\left(k_{2}, s_{2}\right)$, which is in the $k_{1}+k_{2}$ rest frame, we obtain

$$
\begin{aligned}
& \varepsilon_{I}^{0}\left(k_{2}, s_{2}\right)=\frac{1}{m_{2}} \vec{\varepsilon}\left(s_{2}\right) \cdot \vec{k}_{2}, \\
& \vec{\varepsilon}_{I}\left(k_{2}, s_{2}\right)=\vec{\varepsilon}_{I}\left(s_{2}\right)+\frac{\vec{k}_{2}}{m_{2}}\left(\frac{\vec{\varepsilon}_{I}\left(s_{2}\right) \cdot \vec{k}_{2}}{e_{2}+m_{2}}\right) .
\end{aligned}
$$

We can express the above results in a compact form:

$$
\varepsilon_{I}^{\mu}\left(k_{2}, s_{2}\right)=\varepsilon_{I}^{\mu}\left(\bar{P}, s_{2}\right)-\frac{M_{0} k_{2}^{\mu}+m_{2} \bar{P}^{\mu}}{m_{2} M_{0}} \frac{\varepsilon_{I}\left(\bar{P}, s_{2}\right) \cdot k_{2}}{e_{2}+m_{2}} .
$$

Note that we have made use of Eq. (A8) in the above equation, and, consequently, Eq. (A5) is obtained. One can easily check that the above expression for $\varepsilon_{I}\left(k_{2}, s_{2}\right)$ satisfies the well-known relations, $k_{2} \cdot \varepsilon_{I}\left(k_{2}, s_{2}\right)=0$ and $\varepsilon_{I}^{*}\left(k_{2}, s_{2}\right) \cdot \varepsilon_{I}\left(k_{2}, s_{2}^{\prime}\right)=-\delta_{s_{2}, s_{2}^{\prime}}$.

\section{2. $\Gamma$ for the $\left(n, L_{K}, S_{[q q]}^{P}, J_{l}^{P}, J^{P}\right)=\left(n, 0,0^{+}, 0^{+}, 1^{+}\right)$ configuration}

From Eq. (17) the corresponding momentum-space wave-function $\Psi_{n L_{K} S_{[q]]} J_{l}}^{J J_{z}}$ is given by

$$
\begin{aligned}
\Psi_{n s 00}^{1 / 2 J_{z}} & \left(\tilde{p}_{1}, \tilde{p}_{2}, \lambda_{1}, \lambda_{2}\right) \\
= & \left\langle\lambda_{1}\left|\mathcal{R}_{M}^{\dagger}\left(p_{1}^{+}, \vec{p}_{1 \perp}, m_{1}\right)\right| s_{1}\right\rangle\left\langle 0\left|\mathcal{R}_{M}^{\dagger}\left(p_{2}^{+}, \vec{p}_{2 \perp}, m_{2}\right)\right| 0\right\rangle \\
& \times\left\langle\frac{1}{2} 0 ; s_{1} 0 \mid \frac{1}{2} 0 ; \frac{1}{2} J_{z}\right\rangle\langle 00 ; 00 \mid 00 ; 00\rangle \\
& \times \phi_{n 00}\left(x_{1}, x_{2}, k_{1 \perp}, k_{2 \perp}\right) .
\end{aligned}
$$

It can be expressed as 


$$
\begin{aligned}
\Psi_{n s 00}^{1 / 2 J_{z}}\left(\tilde{p}_{1}, \tilde{p}_{2}, \lambda_{1}, \lambda_{2}\right) & =\left\langle\lambda_{1}\left|\mathcal{R}_{M}^{\dagger}\left(p_{1}^{+}, \vec{p}_{1 \perp}, m_{1}\right)\right| s_{1}\right\rangle\left\langle\frac{1}{2} 0 ; s_{1} 0 \mid \frac{1}{2} 0 ; \frac{1}{2} J_{z}\right\rangle \phi_{n 00}\left(x_{1}, x_{2}, k_{1 \perp}, k_{2 \perp}\right) \\
& =\frac{1}{\sqrt{\left(M_{0}+m_{1}\right)^{2}-m_{2}^{2}}} \bar{u}\left(p_{1}, \lambda_{1}\right) \Gamma_{s 00} u\left(\bar{P}, J_{z}\right) \phi_{n s}\left(x_{1}, x_{2}, k_{1 \perp}, k_{2 \perp}\right),
\end{aligned}
$$

with

$$
\Gamma_{s 00}=1,
$$

where we have used Eqs. (A1) and (A3). Putting everything together and boosting $k_{i} \rightarrow p_{i}$ in the LF boost we obtain Eqs. (A13) and (A14).

\section{3. $\Gamma$ for the $\left(n, L_{K}, S_{[q q]}^{P}, J_{l}^{P}, J^{P}\right)=\left(n, 0,1^{+}, 1^{+}, \frac{1}{2}+\right)$ configuration}

From Eq. (17) the corresponding momentum-space wave-function $\Psi_{n L_{K} S_{[q q]} J_{l}}^{1 / 2 J_{z}}$ is given by as

$$
\begin{aligned}
\Psi_{n s 11}^{1 / 2 J_{z}} & \left(\tilde{p}_{1}, \tilde{p}_{2}, \lambda_{1}, \lambda_{2}\right) \\
= & \left\langle\lambda_{1}\left|\mathcal{R}_{M}^{\dagger}\left(p_{1}^{+}, \vec{p}_{1 \perp}, m_{1}\right)\right| s_{1}\right\rangle\left\langle\lambda_{2}\left|\mathcal{R}_{M}^{\dagger}\left(p_{2}^{+}, \vec{p}_{2 \perp}, m_{2}\right)\right| s_{2}\right\rangle \\
& \times\left\langle\frac{1}{2} 1 ; s_{1} J_{l z} \mid \frac{1}{2} 1 ; \frac{1}{2} J_{z}\right\rangle\left\langle 01 ; 0 s_{2} \mid 01 ; 1 J_{l z}\right\rangle \\
& \times \phi_{n 00}\left(x_{1}, x_{2}, k_{1 \perp}, k_{2 \perp}\right) .
\end{aligned}
$$

It can be expressed as

$$
\begin{aligned}
& \Psi_{n s 11}^{1 / 2 J_{z}}\left(\tilde{p}_{1}, \tilde{p}_{2}, \lambda_{1}, \lambda_{2}\right) \\
& =\frac{1}{\sqrt{\left(M_{0}+m_{1}\right)^{2}-m_{2}^{2}}} \bar{u}\left(p_{1}, \lambda_{1}\right) \Gamma_{s 11} u\left(\bar{P}, J_{z}\right) \\
& \quad \times \phi_{n s}\left(x_{1}, x_{2}, k_{1 \perp}, k_{2 \perp}\right),
\end{aligned}
$$

with

$\Gamma_{s 11}=\frac{\gamma_{5}}{\sqrt{3}}\left(\phi_{L F}^{*}\left(p_{2}, \lambda_{2}\right)-\frac{M_{0} \not \not_{2}+m_{2} \overline{P P}}{\bar{P} \cdot p_{2}+m_{2} M_{0}} \frac{\varepsilon_{L F}^{*}\left(p_{2}, \lambda_{2}\right) \cdot \bar{P}}{M_{0}}\right)$,

where we have made use of Eqs. (A1), (A2), (A4)-(A6). In particular using Eqs. (A2), (A5), and (A6), we have

$$
\begin{aligned}
& \left\langle\lambda_{2}\left|\mathcal{R}_{M}^{\dagger}\left(x_{2}, k_{2 \perp}, m_{2}\right)\right| s_{2}\right\rangle \varepsilon_{I}^{* \nu}\left(\bar{P}, s_{2}\right) \\
& =\varepsilon_{L F}^{* \nu}\left(k_{2}, \lambda_{2}\right)-\frac{M_{0} k_{2}^{\nu}+m_{2} \bar{P}^{\nu}}{\left(\bar{P} \cdot k_{2}+m_{2} M_{0}\right)} \frac{\varepsilon_{L F}^{*}\left(k_{2}, \lambda_{2}\right) \cdot \bar{P}}{M_{0}} .
\end{aligned}
$$

Putting everything together and boosting $k_{i} \rightarrow p_{i}$ in the LF boost we obtain Eqs. (A16) and (A17). Using equation of motion, we finally obtain $\Gamma_{s 11}$ as shown in Eq. (29).
4. $\Gamma$ for the $\left(n, L_{K}, S_{[q q]}^{P}, J_{l}^{P}, J^{P}\right)=\left(n, 1,0^{+}, 1^{-}, 1^{-}\right)$

\section{configuration}

From Eq. (17) the corresponding momentum-space wave-function $\Psi_{n L_{K} S_{[q q]} J_{l}}^{J J_{z}}$ is given by

$$
\begin{aligned}
\Psi_{n p 01}^{\frac{1}{2} J_{z}} & \left(\tilde{p}_{1}, \tilde{p}_{2}, \lambda_{1}, \lambda_{2}\right) \\
= & \left\langle\lambda_{1}\left|\mathcal{R}_{M}^{\dagger}\left(p_{1}^{+}, \vec{p}_{1 \perp}, m_{1}\right)\right| s_{1}\right\rangle \\
& \times\left\langle\frac{1}{2} 1 ; s_{1} J_{l z} \mid \frac{1}{2} 1 ; \frac{1}{2} J_{z}\right\rangle\left\langle 10 ; L_{z} 0 \mid 10 ; 1 J_{l z}\right\rangle \\
& \times \phi_{n 1 L_{z}}\left(x_{1}, x_{2}, k_{1 \perp}, k_{2 \perp}\right) .
\end{aligned}
$$

It can be expressed as

$$
\begin{aligned}
& \Psi_{n p 01}^{\frac{1}{2} J_{z}}\left(\tilde{p}_{1}, \tilde{p}_{2}, \lambda_{1}, \lambda_{2}\right) \\
& =\frac{1}{\sqrt{\left(M_{0}+m_{1}\right)^{2}-m_{2}^{2}}} \bar{u}\left(p_{1}, \lambda_{1}\right) \Gamma_{p 01} u\left(\bar{P}, J_{z}\right) \\
& \quad \times \phi_{n p}\left(x_{1}, x_{2}, k_{1 \perp}, k_{2 \perp}\right),
\end{aligned}
$$

with

$$
\Gamma_{p 01}=\frac{\gamma_{5}}{2 \sqrt{3}}\left(\not p_{1}-\not p_{2}-\frac{m_{1}^{2}-m_{2}^{2}-\not p}{M_{0}^{2}}\right),
$$

where Eqs. (A1), (A4), (21), and (A6) have been used. Using equation of motion, we finally obtain $\Gamma_{p 01}$ as shown in Eq. (29).

\section{APPENDIX B: OBTAINING TRANSITION FORM FACTORS}

We shall follow $[15,41]$ to project out various form factors from the transition matrix elements. As in $[15,34,41]$, we consider the $q^{+}=0, \vec{q}_{\perp} \neq \overrightarrow{0}$ case. With the help of the following identities,

$$
\begin{aligned}
\frac{\bar{u}\left(P^{\prime}, J_{z}^{\prime}\right) \gamma^{+} u\left(P, J_{z}\right)}{2 \sqrt{P^{+} P^{\prime+}}} & =\delta_{J_{z}^{\prime} J_{z}}, \\
i \frac{\bar{u}\left(P^{\prime}, J_{z}^{\prime}\right) \sigma^{+\nu} q_{\nu} u\left(P, J_{z}\right)}{2 \sqrt{P^{+} P^{\prime+}}} & =\left(\vec{\sigma} \cdot \vec{q}_{\perp} \sigma^{3}\right)_{J_{z}^{\prime} J_{z},} \\
\frac{\bar{u}\left(P^{\prime}, J_{z}^{\prime}\right) \gamma^{+} \gamma_{5} u\left(P, J_{z}\right)}{2 \sqrt{P^{+} P^{\prime+}}} & =\left(\sigma^{3}\right)_{J_{z}^{\prime} J_{z}}, \\
i \frac{\bar{u}\left(P^{\prime}, J_{z}^{\prime}\right) \sigma^{+\nu} q_{\nu} \gamma_{5} u\left(P, J_{z}\right)}{2 \sqrt{P^{+} P^{\prime+}}} & =\left(\vec{\sigma} \cdot \vec{q}_{\perp}\right)_{J_{z}^{\prime} J_{z},}
\end{aligned}
$$


the matrix elements of $\mathcal{B}_{b}\left(1 / 2^{+}\right) \rightarrow \mathcal{B}_{c}\left(1 / 2^{+}\right)$transition can be expressed as

$$
\begin{aligned}
& \left\langle\mathcal{B}_{c}\left(P^{\prime}, J_{z}^{\prime}\right)\left|V^{+}\right| \mathcal{B}_{b}\left(P, J_{z}\right)\right\rangle \\
& =2 \sqrt{P^{+} P^{\prime+}}\left[f_{1}^{V}\left(q^{2}\right) \delta_{J_{z}^{\prime} J_{z}}+\frac{f_{2}^{V}\left(q^{2}\right)}{M+M^{\prime}}\left(\vec{\sigma} \cdot \vec{q}_{\perp} \sigma^{3}\right)_{J_{z}^{\prime} J_{z}}\right], \\
& \left\langle\mathcal{B}_{c}\left(P^{\prime}, J_{z}^{\prime}\right)\left|A^{+}\right| \mathcal{B}_{b}\left(P, J_{z}\right)\right\rangle \\
& =2 \sqrt{P^{+} P^{\prime+}}\left[g_{1}^{A}\left(q^{2}\right)\left(\sigma^{3}\right)_{J_{z}^{\prime} J_{z}}+\frac{g_{2}^{A}\left(q^{2}\right)}{M+M^{\prime}}\left(\vec{\sigma} \cdot \vec{q}_{\perp}\right)_{J_{z}^{\prime} J_{z}}\right],
\end{aligned}
$$

and similar expressions for the $\mathcal{B}_{b}\left(1 / 2^{+}\right) \rightarrow \mathcal{B}_{c}\left(1 / 2^{-}\right)$case with suitable replacement of $V$ and $A$. Various form factors can be projected out by applying the orthogonality of the corresponding matrices, $\delta_{J_{z} J_{z}^{\prime}},\left(\sigma^{3} \sigma_{\perp}^{i}\right)_{J_{z} J_{z}^{\prime}},\left(\sigma^{3}\right)_{J_{z} J_{z}^{\prime}}$, and $\left(\sigma_{\perp}^{i}\right)_{J_{z} J_{z}^{\prime}}$, under the trace operation:

$$
\begin{aligned}
f_{1}^{V}\left(q^{2}\right) & =\frac{1}{2} \sum_{J_{z}, J_{z}^{\prime}} \delta_{J_{z} J_{z}^{\prime}} \frac{\left\langle\mathcal{B}_{c}\left(P^{\prime}, J_{z}^{\prime}\right)\left|V^{+}\right| \mathcal{B}_{b}\left(P, J_{z}\right)\right\rangle}{2 \sqrt{P^{+} P^{\prime+}}}, \\
\frac{f_{2}^{V}\left(q^{2}\right) q_{\perp}^{i}}{M+M^{\prime}} & =\frac{1}{2} \sum_{J_{z}, J_{z}^{\prime}}\left(\sigma^{3} \sigma_{\perp}^{i}\right)_{J_{z} J_{z}^{\prime}} \frac{\left\langle\mathcal{B}_{c}\left(P^{\prime}, J_{z}^{\prime}\right)\left|V^{+}\right| \mathcal{B}_{b}\left(P, J_{z}\right)\right\rangle}{2 \sqrt{P^{+} P^{\prime+}}}, \\
g_{1}^{A}\left(q^{2}\right) & =\frac{1}{2} \sum_{J_{z}, J_{z}^{\prime}}\left(\sigma^{3}\right)_{J_{z} J_{z}^{\prime}} \frac{\left\langle\mathcal{B}_{c}\left(P^{\prime}, J_{z}^{\prime}\right)\left|A^{+}\right| \mathcal{B}_{b}\left(P, J_{z}\right)\right\rangle}{2 \sqrt{P^{+} P^{\prime+}}}, \\
\frac{g_{2}^{A}\left(q^{2}\right) q_{\perp}^{i}}{M+M^{\prime}} & =\frac{1}{2} \sum_{J_{z}, J_{z}^{\prime}}\left(\sigma_{\perp}^{i}\right)_{J_{z} J_{z}^{\prime}} \frac{\left\langle\mathcal{B}_{c}\left(P^{\prime}, J_{z}^{\prime}\right)\left|A^{+}\right| \mathcal{B}_{b}\left(P, J_{z}\right)\right\rangle}{2 \sqrt{P^{+} P^{\prime+}}}
\end{aligned}
$$

and similar equations for $f_{1,2}^{A}$ and $g_{1,2}^{V}$ in the $\mathcal{B}_{b}\left(1 / 2^{+}\right) \rightarrow$ $\mathcal{B}_{c}\left(1 / 2^{-}\right)$case by suitably replacing $V$ and $A$. Note that due to the condition $q^{+}=0$ we have imposed, the form factors $f_{3}^{V, A}\left(q^{3}\right)$ and $g_{3}^{A, V}\left(q^{3}\right)$ cannot be extracted in this manner. Substituting Eq. (37) to the right-hand side of the above equations, expressions of $\sum_{J_{z}, J_{z}^{\prime}} \delta_{J_{z} J_{z}^{\prime}} \bar{u}\left(\bar{P}^{\prime}, J_{z}^{\prime}\right)(\ldots) u\left(\bar{P}, J_{z}\right)$ and so on occur. They can be further simplified by using the following identities ${ }^{6}$ :

$$
\begin{aligned}
& \frac{1}{2} \sum_{J_{z}, J_{z}^{\prime}} u\left(\bar{P}, J_{z}\right) \delta_{J_{z} J_{z}^{\prime}} \bar{u}\left(\bar{P}^{\prime}, J_{z}^{\prime}\right) \\
& =\frac{1}{4 \sqrt{P^{+} P^{++}}}\left(\bar{P}+M_{0}\right) \gamma^{+}\left(\bar{P}^{\prime}+M_{0}^{\prime}\right), \\
& \frac{1}{2} \sum_{J_{z}, J_{z}^{\prime}} u\left(\bar{P}, J_{z}\right)\left(\sigma^{3} \sigma_{\perp}^{i}\right)_{J_{z} J_{z}} \bar{u}\left(\bar{P}^{\prime}, J_{z}^{\prime}\right) \\
& =-\frac{i}{4 \sqrt{P^{+} P^{\prime+}}}\left(\bar{P}+M_{0}\right) \sigma^{i+}\left(\bar{P}^{\prime}+M_{0}^{\prime}\right), \\
& \frac{1}{2} \sum_{J_{z}, J_{z}^{\prime}} u\left(\bar{P}, J_{z}\right)\left(\sigma^{3}\right)_{J_{z} J_{z}^{\prime}} \bar{u}\left(\bar{P}^{\prime}, J_{z}^{\prime}\right) \\
& =\frac{1}{4 \sqrt{P^{+} P^{\prime+}}}\left(\bar{P}+M_{0}\right) \gamma^{+} \gamma_{5}\left(\bar{P}^{\prime}+M_{0}^{\prime}\right), \\
& \frac{1}{2} \sum_{J_{z}, J_{z}^{\prime}} u\left(\bar{P}, J_{z}\right)\left(\sigma_{\perp}^{i}\right)_{J_{z} J_{z}^{\prime}} \bar{u}\left(\bar{P}^{\prime}, J_{z}^{\prime}\right) \\
& =\frac{i}{4 \sqrt{P^{+} P^{\prime+}}}\left(\bar{P}+M_{0}\right) \sigma^{i+} \gamma_{5}\left(\bar{P}^{\prime}+M_{0}^{\prime}\right) .
\end{aligned}
$$

With the above generic discussions on $\mathcal{B}_{b} \rightarrow \mathcal{B}_{c}$ transition, we are ready to extract the transition form factors: for $\mathcal{B}_{b}\left(1 / 2^{+}\right) \rightarrow \mathcal{B}_{c}\left(1 / 2^{+}\right)$transition, we have

$$
\begin{aligned}
& f_{1}^{V}\left(q^{2}\right)=\int\left\{d \tilde{p}_{2}\right\} \frac{\phi_{n L_{k}^{\prime}}^{\prime *}\left(\left\{x^{\prime}\right\},\left\{k_{\perp}^{\prime}\right\}\right) \phi_{1 L_{K}}\left(\{x\},\left\{k_{\perp}\right\}\right)}{16 P^{+} P^{\prime+} \sqrt{p_{1}^{\prime+} p_{1}^{+}\left(p_{1}^{\prime} \cdot \bar{P}^{\prime}+m_{1}^{\prime} M_{0}^{\prime}\right)\left(p_{1} \cdot \bar{P}+m_{1} M_{0}\right)}} \\
& \times \operatorname{Tr}\left[\left(\bar{P}+M_{0}\right) \gamma^{+}\left(\bar{P}^{\prime}+M_{0}^{\prime}\right) \bar{\Gamma}_{L_{K}^{\prime} S_{[q q]} J_{l}^{\prime}}\left(\not \not_{1}^{\prime}+m_{1}^{\prime}\right) \gamma^{+}\left(\not p_{1}+m_{1}\right) \Gamma_{L_{K} S_{[q q]} J_{l}}\right], \\
& \frac{f_{2}^{V}\left(q^{2}\right) q_{\perp}^{i}}{M+M^{\prime}}=-i \int\left\{d \tilde{p}_{2}\right\} \frac{\phi_{n L_{k}^{\prime}}^{\prime *}\left(\left\{x^{\prime}\right\},\left\{k_{\perp}^{\prime}\right\}\right) \phi_{1 L_{K}}\left(\{x\},\left\{k_{\perp}\right\}\right)}{16 P^{+} P^{\prime+} \sqrt{p_{1}^{\prime+} p_{1}^{+}\left(p_{1}^{\prime} \cdot \bar{P}^{\prime}+m_{1}^{\prime} M_{0}^{\prime}\right)\left(p_{1} \cdot \bar{P}+m_{1} M_{0}\right)}} \\
& \times \operatorname{Tr}\left[\left(\bar{P}+M_{0}\right) \sigma^{i+}\left(\bar{P}^{\prime}+M_{0}^{\prime}\right) \bar{\Gamma}_{L_{K}^{\prime} S_{[q]]} J_{l}^{\prime}}\left(\not p_{1}^{\prime}+m_{1}^{\prime}\right) \gamma^{+}\left(\not p_{1}+m_{1}\right) \Gamma_{\left.L_{K} S_{[q]} J_{l}\right]}\right], \\
& g_{1}^{A}\left(q^{2}\right)=\int\left\{d \tilde{p}_{2}\right\} \frac{\phi_{n L_{k}^{\prime}}^{\prime *}\left(\left\{x^{\prime}\right\},\left\{k_{\perp}^{\prime}\right\}\right) \phi_{1 L_{K}}\left(\{x\},\left\{k_{\perp}\right\}\right)}{16 P^{+} P^{\prime+} \sqrt{p_{1}^{\prime+} p_{1}^{+}\left(p_{1}^{\prime} \cdot \bar{P}^{\prime}+m_{1}^{\prime} M_{0}^{\prime}\right)\left(p_{1} \cdot \bar{P}+m_{1} M_{0}\right)}} \\
& \times \operatorname{Tr}\left[\left(\bar{P}+M_{0}\right) \gamma^{+} \gamma_{5}\left(\bar{P}^{\prime}+M_{0}^{\prime}\right) \bar{\Gamma}_{L_{K}^{\prime} S_{[q]]} J_{l}^{\prime}}\left(\not p_{1}^{\prime}+m_{1}^{\prime}\right) \gamma^{+} \gamma_{5}\left(\not p_{1}+m_{1}\right) \Gamma_{\left.L_{K} S_{[q]} J_{l}\right]}\right], \\
& \frac{g_{2}^{A}\left(q^{2}\right) q_{\perp}^{i}}{M+M^{\prime}}=i \int\left\{d \tilde{p}_{2}\right\} \frac{\phi_{n L_{k}^{\prime}}^{\prime *}\left(\left\{x^{\prime}\right\},\left\{k_{\perp}^{\prime}\right\}\right) \phi_{1 L_{K}}\left(\{x\},\left\{k_{\perp}\right\}\right)}{16 P^{+} P^{\prime+} \sqrt{p_{1}^{\prime+} p_{1}^{+}\left(p_{1}^{\prime} \cdot \bar{P}^{\prime}+m_{1}^{\prime} M_{0}^{\prime}\right)\left(p_{1} \cdot \bar{P}+m_{1} M_{0}\right)}} \\
& \times \operatorname{Tr}\left[\left(\bar{P}+M_{0}\right) \sigma^{i+} \gamma_{5}\left(\bar{P}^{\prime}+M_{0}^{\prime}\right) \bar{\Gamma}_{L_{K}^{\prime} S_{[q q]} J_{l}^{\prime}}\left(\not p_{1}^{\prime}+m_{1}^{\prime}\right) \gamma^{+} \gamma_{5}\left(\not \not 1+m_{1}\right) \Gamma_{L_{K} S_{[q q]} J_{l}}\right],
\end{aligned}
$$

\footnotetext{
${ }^{6}$ These identities can be easily proved by using Eq. (B1), but with $P$ and $P^{\prime}$ replaced by $\bar{P}$ and $\bar{P}^{\prime}$ and with suitable replacements of $J_{z}$ and $J_{z}^{\prime}$.
} 
with $q_{\perp}^{i}=q_{\perp}^{1}$ or $q_{\perp}^{2}$ (no sum over $i$ ), and similarly, for $\mathcal{B}_{b}\left(1 / 2^{+}\right) \rightarrow \mathcal{B}_{c}\left(1 / 2^{-}\right)$transition, we have

$$
\begin{aligned}
& f_{1}^{A}\left(q^{2}\right)=\int\left\{d \tilde{p}_{2}\right\} \frac{\phi_{n L_{k}^{\prime}}^{\prime *}\left(\left\{x^{\prime}\right\},\left\{k_{\perp}^{\prime}\right\}\right) \phi_{1 L_{K}}\left(\{x\},\left\{k_{\perp}\right\}\right)}{16 P^{+} P^{\prime+} \sqrt{p_{1}^{\prime+} p_{1}^{+}\left(p_{1}^{\prime} \cdot \bar{P}^{\prime}+m_{1}^{\prime} M_{0}^{\prime}\right)\left(p_{1} \cdot \bar{P}+m_{1} M_{0}\right)}} \\
& \times \operatorname{Tr}\left[\left(\bar{P}+M_{0}\right) \gamma^{+}\left(\bar{P}^{\prime}+M_{0}^{\prime}\right) \bar{\Gamma}_{L_{K}^{\prime} S_{[q q]} J_{l}^{\prime}}\left(\not \not l_{1}^{\prime}+m_{1}^{\prime}\right) \gamma^{+} \gamma_{5}\left(\not p_{1}+m_{1}\right) \Gamma_{\left.L_{K} S_{[q q]} J_{l}\right]}\right], \\
& \frac{f_{2}^{A}\left(q^{2}\right) q_{\perp}^{i}}{M+M^{\prime}}=-i \int\left\{d \tilde{p}_{2}\right\} \frac{\phi_{n L_{k}^{\prime}}^{\prime *}\left(\left\{x^{\prime}\right\},\left\{k_{\perp}^{\prime}\right\}\right) \phi_{1 L_{K}}\left(\{x\},\left\{k_{\perp}\right\}\right)}{16 P^{+} P^{\prime+} \sqrt{p_{1}^{\prime+} p_{1}^{+}\left(p_{1}^{\prime} \cdot \bar{P}^{\prime}+m_{1}^{\prime} M_{0}^{\prime}\right)\left(p_{1} \cdot \bar{P}+m_{1} M_{0}\right)}} \\
& \times \operatorname{Tr}\left[\left(\bar{P}+M_{0}\right) \sigma^{i+}\left(\bar{P}^{\prime}+M_{0}^{\prime}\right) \bar{\Gamma}_{\left.L_{K}^{\prime} S_{[q q]}\right]_{l}^{\prime}}\left(\not p_{1}^{\prime}+m_{1}^{\prime}\right) \gamma^{+} \gamma_{5}\left(\not p_{1}+m_{1}\right) \Gamma_{\left.L_{K} S_{[q]} J_{l}\right]}\right], \\
& g_{1}^{V}\left(q^{2}\right)=\int\left\{d \tilde{p}_{2}\right\} \frac{\phi_{n L_{k}^{\prime}}^{\prime *}\left(\left\{x^{\prime}\right\},\left\{k_{\perp}^{\prime}\right\}\right) \phi_{1 L_{K}}\left(\{x\},\left\{k_{\perp}\right\}\right)}{16 P^{+} P^{\prime+} \sqrt{p_{1}^{\prime+} p_{1}^{+}\left(p_{1}^{\prime} \cdot \bar{P}^{\prime}+m_{1}^{\prime} M_{0}^{\prime}\right)\left(p_{1} \cdot \bar{P}+m_{1} M_{0}\right)}} \\
& \times \operatorname{Tr}\left[\left(\bar{P}+M_{0}\right) \gamma^{+}\left(\bar{P}^{\prime}+M_{0}^{\prime}\right) \bar{\Gamma}_{L_{K}^{\prime} S_{[q q]} J_{l}^{\prime}}\left(\not \not p_{1}^{\prime}+m_{1}^{\prime}\right) \gamma^{+} \gamma_{5}\left(\not \not_{1}+m_{1}\right) \Gamma_{\left.L_{K} S_{[q q]} J_{l}\right]}\right], \\
& \frac{g_{2}^{A}\left(q^{2}\right) q_{\perp}^{i}}{M+M^{\prime}}=i \int\left\{d \tilde{p}_{2}\right\} \frac{\phi_{n L_{k}^{\prime}}^{*}\left(\left\{x^{\prime}\right\},\left\{k_{\perp}^{\prime}\right\}\right) \phi_{1 L_{K}}\left(\{x\},\left\{k_{\perp}\right\}\right)}{16 P^{+} P^{\prime+} \sqrt{p_{1}^{\prime+} p_{1}^{+}\left(p_{1}^{\prime} \cdot \bar{P}^{\prime}+m_{1}^{\prime} M_{0}^{\prime}\right)\left(p_{1} \cdot \bar{P}+m_{1} M_{0}\right)}} \\
& \times \operatorname{Tr}\left[\left(\bar{P}+M_{0}\right) \sigma^{i+} \gamma_{5}\left(\bar{P}^{\prime}+M_{0}^{\prime}\right) \bar{\Gamma}_{\left.L_{K}^{\prime} S_{[q q]}\right]_{l}^{\prime}}\left(\not \not_{1}^{\prime}+m_{1}^{\prime}\right) \gamma^{+}\left(\not p_{1}+m_{1}\right) \Gamma_{\left.L_{K} S_{[q q]} J_{l}\right]}\right],
\end{aligned}
$$

with $q_{\perp}^{i}=q_{\perp}^{1}$ or $q_{\perp}^{2}$ (no sum over $i$ ). We are now ready to discuss various transitions in more detail.

\section{Form factors for $\mathcal{B}_{b}\left(\overline{\mathbf{3}}_{\mathbf{f}}, \mathbf{1} / \mathbf{2}^{+}\right) \rightarrow \mathcal{B}_{c}\left(\overline{\mathbf{3}}_{\mathbf{f}}, \mathbf{1} / \mathbf{2}^{+}\right)$transition [type (i)]}

We will discuss how to obtain the formulas of form factors of the type (i) transition in this subsection. The $\mathcal{B}_{b}\left(\overline{\mathbf{3}}_{\mathbf{f}}, 1 / 2^{+}\right) \rightarrow$ $\mathcal{B}_{c}\left(\overline{\mathbf{3}}_{\mathbf{f}}, 1 / 2^{+}\right)$transitions involve initial states in $\left(n, L_{K}, S_{[q q]}^{P}, J_{l}^{P}, J^{P}\right)_{b}=\left(1,0,0^{+}, 0^{+}, \frac{1}{2}\right)$ configuration and final states in $\left(n, L_{K}, S_{[q q]}^{P}, J_{l}^{P}, J^{P}\right)_{c}=\left(n, 0,0^{+}, 0^{+}, \frac{1}{2}+\right)$ configurations (with $\left.n=1,2\right)$. In these transitions the scalar diquarks are spectators.

Following Eq. (29),

$$
\Gamma_{L_{K}^{\prime} S_{[q q]}^{\prime} J_{l}^{\prime}}=\Gamma_{L_{K} S_{[q q]} J_{l}}=\Gamma_{s 00}=1,
$$

and (37), we have

$$
\begin{aligned}
\left\langle\mathcal{B}_{c}\left(P^{\prime}, J_{z}^{\prime}\right)\left|\bar{c} \gamma^{+}\left(1-\gamma_{5}\right) b\right| \mathcal{B}_{b}\left(P, J_{z}\right)\right\rangle= & \int\left\{d \tilde{p}_{2}\right\} \frac{\phi_{n L_{k}^{\prime}}^{\prime *}\left(\left\{x^{\prime}\right\},\left\{k_{\perp}^{\prime}\right\}\right) \phi_{1 L_{K}}\left(\{x\},\left\{k_{\perp}\right\}\right)}{2 \sqrt{p_{1}^{\prime+} p_{1}^{+}\left(p_{1}^{\prime} \cdot \bar{P}^{\prime}+m_{1}^{\prime} M_{0}^{\prime}\right)\left(p_{1} \cdot \bar{P}+m_{1} M_{0}\right)}} \\
& \times \bar{u}\left(\bar{P}^{\prime}, J_{z}^{\prime}\right)\left(\not p_{1}^{\prime}+m_{1}^{\prime}\right) \gamma^{\mu}\left(1-\gamma_{5}\right)\left(\not p_{1}+m_{1}\right) u\left(\bar{P}, J_{z}\right),
\end{aligned}
$$

for the type (i) transition. By using Eq. (B5) the transition form factors are given by

$$
\begin{aligned}
f_{1}^{V}\left(q^{2}\right)= & \int \frac{d x_{2} d^{2} k_{2 \perp}}{2(2 \pi)^{3}} \frac{\phi_{n s}^{\prime *}\left(\left\{x^{\prime}\right\},\left\{k_{\perp}^{\prime}\right\}\right) \phi_{1 s}\left(\{x\},\left\{k_{\perp}\right\}\right)}{16 P^{+} P^{\prime+} \sqrt{x_{1}^{\prime+} x_{1}^{+}\left(p_{1}^{\prime} \cdot \bar{P}^{\prime}+m_{1}^{\prime} M_{0}^{\prime}\right)\left(p_{1} \cdot \bar{P}+m_{1} M_{0}\right)}} \\
& \times \operatorname{Tr}\left[\left(\bar{P}+M_{0}\right) \gamma^{+}\left(\bar{P}^{\prime}+M_{0}^{\prime}\right)\left(\not p_{1}^{\prime}+m_{1}^{\prime}\right) \gamma^{+}\left(\not p_{1}+m_{1}\right)\right], \\
\frac{f_{2}^{V}\left(q^{2}\right) q_{\perp}^{i}}{M+M^{\prime}}= & -i \int \frac{d x_{2} d^{2} k_{2 \perp}}{2(2 \pi)^{3}} \frac{\phi_{n s}^{\prime *}\left(\left\{x^{\prime}\right\},\left\{k_{\perp}^{\prime}\right\}\right) \phi_{1 s}\left(\{x\},\left\{k_{\perp}\right\}\right)}{16 P^{+} P^{\prime+} \sqrt{x_{1}^{\prime+} x_{1}^{+}\left(p_{1}^{\prime} \cdot \bar{P}^{\prime}+m_{1}^{\prime} M_{0}^{\prime}\right)\left(p_{1} \cdot \bar{P}+m_{1} M_{0}\right)}} \\
& \times \operatorname{Tr}\left[\left(\bar{P}+M_{0}\right) \sigma^{i+}\left(\bar{P}^{\prime}+M_{0}^{\prime}\right)\left(\not p_{1}^{\prime}+m_{1}^{\prime}\right) \gamma^{+}\left(\not p_{1}+m_{1}\right)\right], \\
g_{1}^{A}\left(q^{2}\right)= & \int \frac{d x_{2} d^{2} k_{2 \perp}}{2(2 \pi)^{3}} \frac{\phi_{n s}^{\prime *}\left(\left\{x^{\prime}\right\},\left\{k_{\perp}^{\prime}\right\}\right) \phi_{1 s}\left(\{x\},\left\{k_{\perp}\right\}\right)}{16 P^{+} P^{\prime+} \sqrt{x_{1}^{\prime+} x_{1}^{+}\left(p_{1}^{\prime} \cdot \bar{P}^{\prime}+m_{1}^{\prime} M_{0}^{\prime}\right)\left(p_{1} \cdot \bar{P}+m_{1} M_{0}\right)}} \\
& \times \operatorname{Tr}\left[\left(\bar{P}+M_{0}\right) \gamma^{+} \gamma_{5}\left(\bar{P}^{\prime}+M_{0}^{\prime}\right)\left(\not \not_{1}^{\prime}+m_{1}^{\prime}\right) \gamma^{+} \gamma_{5}\left(\not p_{1}+m_{1}\right)\right], \\
\left.M+M^{\prime}\right) q_{\perp}^{i}= & i \int \frac{d x_{2} d^{2} k_{2 \perp}}{2(2 \pi)^{3}} \frac{\phi_{n s}^{\prime *}\left(\left\{x^{\prime}\right\},\left\{k_{\perp}^{\prime}\right\}\right) \phi_{1 s}\left(\{x\},\left\{k_{\perp}\right\}\right)}{16 P^{+} P^{\prime+} \sqrt{x_{1}^{\prime+} x_{1}^{+}\left(p_{1}^{\prime} \cdot \bar{P}^{\prime}+m_{1}^{\prime} M_{0}^{\prime}\right)\left(p_{1} \cdot \bar{P}+m_{1} M_{0}\right)}} \\
& \times \operatorname{Tr}\left[\left(\bar{P}+M_{0}\right) \sigma^{i+} \gamma_{5}\left(\bar{P}^{\prime}+M_{0}^{\prime}\right)\left(\not p_{1}^{\prime}+m_{1}^{\prime}\right) \gamma^{+} \gamma_{5}\left(\not \not p_{1}+m_{1}\right)\right] .
\end{aligned}
$$

with $q_{\perp}^{i}=q_{\perp}^{1}$ or $q_{\perp}^{2}$ (no sum over $i$ ). 
It is straightforward to work out the traces in $f_{1,2}^{V}\left(q^{2}\right)$ as shown in the above equation and obtain [15]

$$
\begin{aligned}
& \frac{1}{8 P^{+} P^{\prime+}} \operatorname{Tr}\left[\left(\bar{P}+M_{0}\right) \gamma^{+}\left(\bar{P}^{\prime}+M_{0}^{\prime}\right)\left(\not \not p_{1}^{\prime}+m_{1}^{\prime}\right) \gamma^{+}\left(\not p_{1}+m_{1}\right)\right] \\
& =-\left(p_{1}-x_{1} \bar{P}\right) \cdot\left(p_{1}^{\prime}-x_{1}^{\prime} \bar{P}^{\prime}\right)+\left(x_{1} M_{0}+m_{1}\right)\left(x_{1}^{\prime} M_{0}^{\prime}+m_{1}^{\prime}\right), \\
& \frac{i}{8 P^{+} P^{\prime+}} \operatorname{Tr}\left[\left(\bar{P}+M_{0}\right) \sigma^{i+}\left(\bar{P}^{\prime}+M_{0}^{\prime}\right)\left(\not p_{1}^{\prime}+m_{1}^{\prime}\right) \gamma^{+}\left(\not \not_{1}+m_{1}\right)\right] \\
& =\left(m_{1}^{\prime}+x_{1}^{\prime} M_{0}^{\prime}\right)\left(p_{\perp}^{i}-x_{1} \bar{P}_{\perp}^{i}\right)-\left(m_{1}+x_{1} M_{0}\right)\left(p_{\perp}^{\prime i}-x_{1}^{\prime} \bar{P}_{\perp}^{\prime i}\right),
\end{aligned}
$$

for $i=1,2$, where uses of $\bar{P}^{(\prime)+}=P^{(\prime)+}, \bar{P}_{\perp}^{(\prime) i}=P_{\perp}^{(\prime) i}$, $p_{1}^{(\prime)+}=x^{(\prime)} P^{(\prime)+}, p_{1 \perp}^{(\prime) i}=x^{(\prime)} P_{\perp}^{(\prime) i}+k_{1 \perp}^{(\prime) i}$ have been made. Similarly the traces in $g_{1}^{A}\left(q^{2}\right)$ and $g_{2}^{A}\left(q^{2}\right)$ can be obtained by replacing $m_{1}^{\prime} \rightarrow-m_{1}^{\prime}, M_{0}^{\prime} \rightarrow-M_{0}^{\prime}$ in the above traces and with an additional overall minus sign. With the help of Eq. (23) the above form factors can be expressed in terms of the internal variables via [15]

$$
\begin{aligned}
& p_{1} \cdot \bar{P}=e_{1} M_{0}=\frac{m_{1}^{2}+x_{1}^{2} M_{0}^{2}+k_{1 \perp}^{2}}{2 x_{1}}, \\
& p_{1}^{\prime} \cdot \bar{P}^{\prime}=e_{1}^{\prime} M_{0}^{\prime}=\frac{m_{1}^{\prime 2}+x_{1}^{2} M_{0}^{\prime 2}+k_{1 \perp}^{\prime 2}}{2 x_{1}}, \\
& \left(p_{1}-x_{1} \bar{P}\right) \cdot\left(p_{1}^{\prime}-x_{1}^{\prime} \bar{P}^{\prime}\right)=-k_{1 \perp} \cdot k_{1 \perp}^{\prime}, \\
& p_{\perp}^{(\prime) i}-x_{1} \bar{P}_{\perp}^{(\prime) i}=k_{1 \perp}^{(\prime) i},
\end{aligned}
$$

where $k_{1 \perp} \cdot k_{1 \perp}^{\prime}$ is a scalar product in two-dimensional space. The obtained form factors are shown in Eq. (39).

\section{Form factors for $\mathcal{B}_{b}\left(6_{\mathrm{f}}, \mathbf{1} / \mathbf{2}^{+}\right) \rightarrow \mathcal{B}_{c}\left(\mathbf{6}_{\mathrm{f}}, \mathbf{1} / \mathbf{2}^{+}\right)$ transition [type (ii)]}

We will discuss how to obtain the formulas of form factors of the type (ii) transition in this subsection. The $\mathcal{B}_{b}\left(\mathbf{6}_{\mathbf{f}}, 1 / 2^{+}\right) \rightarrow \mathcal{B}_{c}\left(\mathbf{6}_{\mathbf{f}}, 1 / 2^{+}\right)$transitions involve initial states in $\left(n, L_{K}, S_{[q q]}^{P}, J_{l}^{P}, J^{P}\right)_{b}=\left(1,0,1^{+}, 1^{+}, \frac{1}{2}{ }^{+}\right)$configuration and final states in $\left(n, L_{K}, S_{[q q]}^{P}, J_{l}^{P}, J^{P}\right)_{c}=$ $\left(n, 0,1^{+}, 1^{+}, \frac{1}{2}^{+}\right)$configurations (with $\left.n=1,2\right)$. In these transitions the axial-vector diquarks are spectators.

Following Eqs. (29) and (37) we have

$$
\begin{aligned}
& \left\langle\mathcal{B}_{c}\left(P^{\prime}, J_{z}^{\prime}\right)\left|\bar{c} \gamma^{\mu}\left(1-\gamma_{5}\right) b\right| \mathcal{B}_{b}\left(P, J_{z}\right)\right\rangle \\
& =\int\left\{d \tilde{p}_{2}\right\} \frac{\phi_{n L_{k}^{\prime}}^{\prime *}\left(\left\{x^{\prime}\right\},\left\{k_{\perp}^{\prime}\right\}\right) \phi_{1 L_{K}}\left(\{x\},\left\{k_{\perp}\right\}\right)}{2 \sqrt{p_{1}^{\prime+} p_{1}^{+}\left(p_{1}^{\prime} \cdot \bar{P}^{\prime}+m_{1}^{\prime} M_{0}^{\prime}\right)\left(p_{1} \cdot \bar{P}+m_{1} M_{0}\right)}} \\
& \times \bar{u}\left(\bar{P}^{\prime}, J_{z}^{\prime}\right) \bar{\Gamma}_{s 11}^{\prime}\left(\not p_{1}^{\prime}+m_{1}^{\prime}\right) \gamma^{\mu}\left(1-\gamma_{5}\right)\left(\not \phi_{1}+m_{1}\right) \Gamma_{s 11} u\left(\bar{P}, J_{z}\right),
\end{aligned}
$$

with

$$
\begin{aligned}
\Gamma_{s 11} & =\frac{\gamma_{5}}{\sqrt{3}}\left(\phi_{L F}^{*}\left(p_{2}, \lambda_{2}\right)-\frac{M_{0}+m_{1}+m_{2}}{\bar{P} \cdot p_{2}+m_{2} M_{0}} \varepsilon_{L F}^{*}\left(p_{2}, \lambda_{2}\right) \cdot \bar{P}\right) \\
& \equiv \varepsilon_{L F \mu}^{*}\left(p_{2}, \lambda_{2}\right) \Gamma_{s 11}^{\mu}, \\
\bar{\Gamma}_{s 11}^{\prime} & =\frac{\gamma_{5}}{\sqrt{3}}\left(\phi_{L F}\left(p_{2}, \lambda_{2}\right)+\frac{M_{0}^{\prime}+m_{1}^{\prime}+m_{2}}{\bar{P}^{\prime} \cdot p_{2}+m_{2} M_{0}^{\prime}} \varepsilon_{L F}\left(p_{2}, \lambda_{2}\right) \cdot \bar{P}^{\prime}\right) \\
& \equiv \varepsilon_{L F \mu}\left(p_{2}, \lambda_{2}\right) \bar{\Gamma}_{s 11}^{\prime \mu},
\end{aligned}
$$

where we have made use of the fact that the diquark is a spectator of the transition. By using Eq. (B5) the transition form factors for the $\mathcal{B}_{b}\left(\mathbf{6}_{\mathbf{f}}, 1 / 2^{+}\right) \rightarrow \mathcal{B}_{c}\left(\mathbf{6}_{\mathbf{f}}, 1 / 2^{+}\right)$case are given by:

$$
\begin{aligned}
f_{1}^{V}\left(q^{2}\right)= & \left.\int\left\{d \tilde{p}_{2}\right\} \frac{\phi_{n L_{k}^{\prime}}^{\prime *}\left(\left\{x^{\prime}\right\},\left\{k_{\perp}^{\prime}\right\}\right) \phi_{1 L_{K}}\left(\{x\},\left\{k_{\perp}\right\}\right)}{16 P^{+} P^{\prime+} \sqrt{p_{1}^{\prime+} p_{1}^{+}\left(p_{1}^{\prime} \cdot \bar{P}^{\prime}+m_{1}^{\prime} M_{0}^{\prime}\right)\left(p_{1} \cdot \bar{P}+m_{1} M_{0}\right)}}\right]\left(-g_{\mu \nu}+\frac{p_{2 \mu} p_{2 \nu}}{m_{2}^{2}}\right) \\
& \times \operatorname{Tr}\left[\left(\bar{P}+M_{0}\right) \gamma^{+}\left(\bar{P}^{\prime}+M_{0}^{\prime}\right) \bar{\Gamma}_{s 11}^{\prime \mu}\left(\not p_{1}^{\prime}+m_{1}^{\prime}\right) \gamma^{+}\left(\not p_{1}+m_{1}\right) \Gamma_{s 11}^{\nu}\right], \\
\frac{f_{2}^{V}\left(q^{2}\right) q_{\perp}^{i}}{M+M^{\prime}}= & \left.-i \int\left\{d \tilde{p}_{2}\right\} \frac{\phi_{n L_{k}^{\prime}}^{\prime *}\left(\left\{x^{\prime}\right\},\left\{k_{\perp}^{\prime}\right\}\right) \phi_{1 L_{K}}\left(\{x\},\left\{k_{\perp}\right\}\right)}{16 P^{+} P^{\prime+} \sqrt{p_{1}^{\prime+} p_{1}^{+}\left(p_{1}^{\prime} \cdot \bar{P}^{\prime}+m_{1}^{\prime} M_{0}^{\prime}\right)\left(p_{1} \cdot \bar{P}+m_{1} M_{0}\right)}}\right]\left(-g_{\mu \nu}+\frac{p_{2 \mu} p_{2 \nu}}{m_{2}^{2}}\right) \\
& \times \operatorname{Tr}\left[\left(\bar{P}+M_{0}\right) \sigma^{i+}\left(\bar{P}^{\prime}+M_{0}^{\prime}\right) \bar{\Gamma}_{s 11}^{\prime \mu}\left(\not p_{1}^{\prime}+m_{1}^{\prime}\right) \gamma^{+}\left(\not p_{1}+m_{1}\right) \Gamma_{s 11}^{\nu}\right], \\
g_{1}^{A}\left(q^{2}\right)= & \left.\int\left\{d \tilde{p}_{2}\right\} \frac{\phi_{n L_{k}^{\prime}}^{\prime *}\left(\left\{x^{\prime}\right\},\left\{k_{\perp}^{\prime}\right\}\right) \phi_{1 L_{K}}\left(\{x\},\left\{k_{\perp}\right\}\right)}{\left.16 P^{+} P^{\prime+} \sqrt{p_{1}^{\prime+} p_{1}^{+}\left(p_{1}^{\prime} \cdot \bar{P}^{\prime}+m_{1}^{\prime} M_{0}^{\prime}\right)\left(p_{1} \cdot \bar{P}+m_{1} M_{0}\right)}\right]}\right]\left(-g_{\mu \nu}+\frac{p_{2 \mu} p_{2 \nu}}{m_{2}^{2}}\right) \\
& \times \operatorname{Tr}\left[\left(\bar{P}+M_{0}\right) \gamma^{+} \gamma_{5}\left(\bar{P}^{\prime}+M_{0}^{\prime}\right) \bar{\Gamma}_{s 11}^{\prime \mu}\left(\not p_{1}^{\prime}+m_{1}^{\prime}\right) \gamma^{+} \gamma_{5}\left(\not p_{1}+m_{1}\right) \Gamma_{s 11}^{\nu}\right], \\
\frac{g_{2}^{A}\left(q^{2}\right) q_{\perp}^{i}=}{M+M^{\prime}} & \left.i \int\left\{d \tilde{p}_{2}\right\} \frac{\phi_{n L_{k}^{\prime}}^{\prime *}\left(\left\{x^{\prime}\right\},\left\{k_{\perp}^{\prime}\right\}\right) \phi_{1 L_{K}}\left(\{x\},\left\{k_{\perp}\right\}\right)}{16 P^{+} P^{\prime+} \sqrt{p_{1}^{\prime+} p_{1}^{+}\left(p_{1}^{\prime} \cdot \bar{P}^{\prime}+m_{1}^{\prime} M_{0}^{\prime}\right)\left(p_{1} \cdot \bar{P}+m_{1} M_{0}\right)}}\right]\left(-g_{\mu \nu}+\frac{p_{2 \mu} p_{2 \nu}}{m_{2}^{2}}\right) \\
& \times \operatorname{Tr}\left[\left(\bar{P}+M_{0}\right) \sigma^{i+} \gamma_{5}\left(\bar{P}^{\prime}+M_{0}^{\prime}\right) \bar{\Gamma}_{s 11}^{\prime \mu}\left(\not p_{1}^{\prime}+m_{1}^{\prime}\right) \gamma^{+} \gamma_{5}\left(\not p_{1}+m_{1}\right) \Gamma_{s 11}^{\nu}\right],
\end{aligned}
$$


with $q_{\perp}^{i}=q_{\perp}^{1}$ or $q_{\perp}^{2}$ (no sum over $i$ ). As one can see the traces are rather complicate. To simplify the derivations, we choose to work in the $\vec{P}_{\perp}=0$ frame. After working out the traces and making use of Eq. (23), we obtain the form factors as shown in Eq. (40).

\section{Form factors for $\mathcal{B}_{b}\left(\overline{\mathbf{3}}_{\mathrm{f}}, 1 / 2^{+}\right) \rightarrow \mathcal{B}_{c}\left(\overline{\mathbf{3}}_{\mathrm{f}}, 1 / 2^{-}\right)$ transition [type (iii)]}

We will discuss how to obtain the formulas of form factors of the type (iii) transition in this subsection. The $\mathcal{B}_{b}\left(\overline{\mathbf{3}}_{\mathbf{f}}, 1 / 2^{+}\right) \rightarrow \mathcal{B}_{c}\left(\overline{\mathbf{3}}_{\mathbf{f}}, 1 / 2^{-}\right)$transitions involve initial states in $\left(n, L_{K}, S_{[q q]}^{P}, J_{l}^{P}, J^{P}\right)_{b}=\left(1,0,0^{+}, 0^{+}, \frac{1}{2}+\right)$ configuration and final states in $\left(n, L_{K}, S_{[q q]}^{P}, J_{l}^{P}, J^{P}\right)_{c}=$ $\left(n, 1,0^{+}, 1^{-}, \frac{1}{2}^{-}\right)$configurations (with $\left.n=1,2\right)$. In these transitions, the scalar diquarks are spectators.
Following Eqs. (29) and (37), we have,

$$
\begin{aligned}
& \left\langle\mathcal{B}_{c}\left(P^{\prime}, J_{z}^{\prime}\right)\left|\bar{c} \gamma^{+}\left(1-\gamma_{5}\right) b\right| \mathcal{B}_{b}\left(P, J_{z}\right)\right\rangle \\
& =\int\left\{d \tilde{p}_{2}\right\} \frac{\phi_{n L_{k}^{\prime}}^{\prime *}\left(\left\{x^{\prime}\right\},\left\{k_{\perp}^{\prime}\right\}\right) \phi_{1 L_{K}}\left(\{x\},\left\{k_{\perp}\right\}\right)}{2 \sqrt{p_{1}^{\prime+} p_{1}^{+}\left(p_{1}^{\prime} \cdot \bar{P}^{\prime}+m_{1}^{\prime} M_{0}^{\prime}\right)\left(p_{1} \cdot \bar{P}+m_{1} M_{0}\right)}} \\
& \quad \times \bar{u}\left(\bar{P}^{\prime}, J_{z}^{\prime}\right) \bar{\Gamma}_{p 01}^{\prime}\left(\not p_{1}^{\prime}+m_{1}^{\prime}\right) \gamma^{\mu}\left(1-\gamma_{5}\right)\left(\not \not_{1}+m_{1}\right) u\left(\bar{P}, J_{z}\right),
\end{aligned}
$$

with

$$
\begin{aligned}
& \Gamma_{L_{K} S_{[q q]} J_{l}}=\Gamma_{s 00}=1, \\
& \bar{\Gamma}_{L_{K}^{\prime} S_{[q q]}^{\prime} J_{l}^{\prime}}=\bar{\Gamma}_{p 01}^{\prime}=\frac{\gamma_{5}}{2 \sqrt{3}}\left(\not p_{1}^{\prime}-\not p_{2}+\frac{m_{1}^{\prime 2}-m_{2}^{2}}{M_{0}^{\prime}}\right),
\end{aligned}
$$

for the $\mathcal{B}_{b}\left(\overline{\mathbf{3}}_{\mathbf{f}}, 1 / 2^{+}\right) \rightarrow \mathcal{B}_{c}\left(\overline{\mathbf{3}}_{\mathbf{f}}, 1 / 2^{-}\right)$transition [type (iii)]. By using Eq. (B6) we obtain the transition form factors:

$$
\begin{aligned}
f_{1}^{A}\left(q^{2}\right)= & \int \frac{d x_{2} d^{2} k_{2 \perp}}{2(2 \pi)^{3}} \frac{\phi_{n p}^{*}\left(\left\{x^{\prime}\right\},\left\{k_{\perp}^{\prime}\right\}\right) \phi_{1 s}\left(\{x\},\left\{k_{\perp}\right\}\right)}{16 P^{+} P^{\prime+} \sqrt{x_{1}^{\prime+} x_{1}^{+}\left(p_{1}^{\prime} \cdot \bar{P}^{\prime}+m_{1}^{\prime} M_{0}^{\prime}\right)\left(p_{1} \cdot \bar{P}+m_{1} M_{0}\right)}} \\
& \times \operatorname{Tr}\left[\left(\bar{P}+M_{0}\right) \gamma^{+}\left(\bar{P}^{\prime}+M_{0}^{\prime}\right) \bar{\Gamma}_{p 01}^{\prime}\left(\not p_{1}^{\prime}+m_{1}^{\prime}\right) \gamma^{+} \gamma_{5}\left(\not p_{1}+m_{1}\right)\right], \\
\frac{f_{2}^{A}\left(q^{2}\right) q_{\perp}^{i}}{M+M^{\prime}}= & -i \int \frac{d x_{2} d^{2} k_{2 \perp}}{2(2 \pi)^{3}} \frac{\phi_{n p}^{\prime *}\left(\left\{x^{\prime}\right\},\left\{k_{\perp}^{\prime}\right\}\right) \phi_{1 s}\left(\{x\},\left\{k_{\perp}\right\}\right)}{16 P^{+} P^{\prime+} \sqrt{x_{1}^{\prime+} x_{1}^{+}\left(p_{1}^{\prime} \cdot \bar{P}^{\prime}+m_{1}^{\prime} M_{0}^{\prime}\right)\left(p_{1} \cdot \bar{P}+m_{1} M_{0}\right)}} \\
& \times \operatorname{Tr}\left[\left(\bar{P}+M_{0}\right) \sigma^{i+}\left(\bar{P}^{\prime}+M_{0}^{\prime}\right) \bar{\Gamma}_{p 01}^{\prime}\left(\not p_{1}^{\prime}+m_{1}^{\prime}\right) \gamma^{+} \gamma_{5}\left(\not p_{1}+m_{1}\right)\right], \\
g_{1}^{V}\left(q^{2}\right)= & \int \frac{d x_{2} d^{2} k_{2 \perp}}{2(2 \pi)^{3}} \frac{\phi_{n p}^{\prime *}\left(\left\{x^{\prime}\right\},\left\{k_{\perp}^{\prime}\right\}\right) \phi_{1 s}\left(\{x\},\left\{k_{\perp}\right\}\right)}{16 P^{+} P^{\prime+} \sqrt{x_{1}^{\prime+} x_{1}^{+}\left(p_{1}^{\prime} \cdot \bar{P}^{\prime}+m_{1}^{\prime} M_{0}^{\prime}\right)\left(p_{1} \cdot \bar{P}+m_{1} M_{0}\right)}} \\
\frac{g_{2}^{V}\left(q^{2}\right) q_{\perp}^{i}}{M+M^{\prime}}= & i \int \frac{d x_{2} d^{2} k_{2 \perp}}{2(2 \pi)^{3}} \frac{\phi_{n p}^{\prime *}\left(\left\{x^{\prime}\right\},\left\{k_{\perp}^{\prime}\right\}\right) \phi_{1 s}\left(\{x\},\left\{k_{\perp}\right\}\right)}{16 P^{+} P^{\prime+} \sqrt{x_{1}^{\prime+} x_{1}^{+}\left(p_{1}^{\prime} \cdot \bar{P}^{\prime}+m_{1}^{\prime} M_{0}^{\prime}\right)\left(p_{1} \cdot \bar{P}+m_{1} M_{0}\right)}} \\
& \times \operatorname{Tr}\left[\left(\bar{P}+M_{0}\right) \sigma^{i+} \gamma_{5}\left(\bar{P}^{\prime}+M_{0}^{\prime}\right) \bar{\Gamma}_{p 01}^{\prime}\left(\not p_{1}^{\prime}+m_{1}^{\prime}\right) \gamma^{+}\left(\not \not p 1+m_{1}\right)\right] .
\end{aligned}
$$

with $q_{\perp}^{i}=q_{\perp}^{1}$ or $q_{\perp}^{2}$ (no sum over $i$ ). To simplify the derivations, we choose to work in the $\vec{P}_{\perp}=0$ frame. After working out traces and making use of Eq. (23), we obtain the form factors as shown in Eq. (51).

[1] M. Tanabashi et al. (Particle Data Group), Review of particle physics, Phys. Rev. D 98, 030001 (2018).

[2] R. Aaij et al. (LHCb Collaboration), Study of the $D^{0} p$ amplitude in $\Lambda_{b}^{0} \rightarrow D^{0} p \pi^{-}$decays, J. High Energy Phys. 05 (2017) 030.

[3] R. Aaij et al. (LHCb Collaboration), Observation of Five New Narrow $\Omega_{c}^{0}$ States Decaying to $\Xi_{c}^{+} K^{-}$, Phys. Rev. Lett. 118, 182001 (2017).
[4] H. Y. Cheng and C. W. Chiang, Quantum numbers of $\Omega_{c}$ states and other charmed baryons, Phys. Rev. D 95, 094018 (2017).

[5] H. X. Chen, Q. Mao, W. Chen, A. Hosaka, X. Liu, and S. L. Zhu, Decay properties of $P$-wave charmed baryons from light-cone QCD sum rules, Phys. Rev. D 95, 094008 (2017).

[6] M. Karliner and J. L. Rosner, Very narrow excited $\Omega_{c}$ baryons, Phys. Rev. D 95, 114012 (2017). 
[7] K. L. Wang, L. Y. Xiao, X. H. Zhong, and Q. Zhao, Understanding the newly observed $\Omega_{c}$ states through their decays, Phys. Rev. D 95, 116010 (2017).

[8] M. Padmanath and N. Mathur, Quantum Numbers of Recently Discovered $\Omega_{c}^{0}$ Baryons from Lattice QCD, Phys. Rev. Lett. 119, 042001 (2017).

[9] Z. G. Wang, Analysis of $\Omega_{c}(3000), \Omega_{c}(3050), \Omega_{c}(3066)$, $\Omega_{c}(3090)$ and $\Omega_{c}(3119)$ with QCD sum rules, Eur. Phys. J. C 77, 325 (2017).

[10] B. Chen and X. Liu, New $\Omega_{c}^{0}$ baryons discovered by LHCb as the members of $1 P$ and $2 S$ states, Phys. Rev. D 96, 094015 (2017).

[11] S. S. Agaev, K. Azizi, and H. Sundu, On the nature of the newly discovered $\Omega$ states, Europhys. Lett. 118, 61001 (2017).

[12] R. Aaij et al. (LHCb Collaboration), Study of the kinematic dependences of $\Lambda_{b}^{0}$ production in $p p$ collisions and a measurement of the $\Lambda_{b}^{0} \rightarrow \Lambda_{c}^{+} \pi^{-}$branching fraction, J. High Energy Phys. 08 (2014) 143.

[13] R. Aaij et al. (LHCb Collaboration), Searches for $\Lambda_{b}^{0}$ and $\Xi_{b}^{0}$ decays to $K_{\mathrm{S}}^{0} p \pi^{-}$and $K_{\mathrm{S}}^{0} p K^{-}$final states with first observation of the $\Lambda_{b}^{0} \rightarrow K_{\mathrm{S}}^{0} p \pi^{-}$decay, J. High Energy Phys. 04 (2014) 087.

[14] R. Aaij et al. (LHCb Collaboration), Study of Beauty Hadron Decays into Pairs of Charm Hadrons, Phys. Rev. Lett. 112, 202001 (2014).

[15] H. Y. Cheng, C. K. Chua, and C. W. Hwang, Light front approach for heavy pentaquark transitions, Phys. Rev. D 70, 034007 (2004).

[16] T. Mannel and W. Roberts, Nonleptonic $\Lambda_{b}$ decays at colliders, Z. Phys. C 59, 179 (1993).

[17] H. Y. Cheng, Nonleptonic weak decays of bottom baryons, Phys. Rev. D 56, 2799 (1997).

[18] M. A. Ivanov, J. G. Korner, V. E. Lyubovitskij, and A. G. Rusetsky, Exclusive nonleptonic bottom to charm baryon decays including nonfactorizable contributions, Mod. Phys. Lett. A 13, 181 (1998).

[19] M. A. Ivanov, J. G. Korner, V. E. Lyubovitskij, and A. G. Rusetsky, Exclusive nonleptonic decays of bottom and charm baryons in a relativistic three quark model: Evaluation of nonfactorizing diagrams, Phys. Rev. D 57, 5632 (1998).

[20] A. K. Giri, L. Maharana, and R. Mohanta, Two-body nonleptonic $\Lambda_{b}$ decays with $1 / M_{Q}$ corrections, Mod. Phys. Lett. A 13, 23 (1998).

[21] Fayyazuddin and Riazuddin, Two-body nonleptonic $\Lambda_{b}$ decays in quark model with factorization ansatz, Phys. Rev. D 58, 014016 (1998).

[22] R. Mohanta, A. K. Giri, M. P. Khanna, M. Ishida, S. Ishida, and M. Oda, Hadronic weak decays of $\Lambda_{b}$ baryon in the covariant oscillator quark model, Prog. Theor. Phys. 101, 959 (1999).

[23] H. H. Shih, S.C. Lee, and H. N. Li, Applicability of perturbative QCD to $\Lambda_{b} \rightarrow \Lambda_{c}$ decays, Phys. Rev. D 61, 114002 (2000).

[24] C. Albertus, E. Hernandez, and J. Nieves, Nonrelativistic constituent quark model and HQET combined study of semileptonic decays of $\Lambda_{b}$ and $\Xi_{b}$ baryons, Phys. Rev. D 71, 014012 (2005).
[25] H. W. Ke, X. Q. Li, and Z. T. Wei, Diquarks and $\Lambda_{b} \rightarrow \Lambda_{c}$ weak decays, Phys. Rev. D 77, 014020 (2008).

[26] H. W. Ke, X. H. Yuan, X. Q. Li, Z. T. Wei, and Y. X. Zhang, $\Sigma_{b} \rightarrow \Sigma_{c}$ and $\Omega_{b} \rightarrow \Omega_{c}$ weak decays in the light-front quark model, Phys. Rev. D 86, 114005 (2012).

[27] W. Detmold, C. Lehner, and S. Meinel, $\Lambda_{b} \rightarrow p \ell^{-} \bar{\nu}_{\ell}$ and $\Lambda_{b} \rightarrow \Lambda_{c} \ell^{-} \bar{\nu}_{\ell}$ form factors from lattice QCD with relativistic heavy quarks, Phys. Rev. D 92, 034503 (2015).

[28] J. Zhu, Z. T. Wei, and H. W. Ke, The semi-leptonic and nonleptonic weak decays of $\Lambda_{b}^{0}$, arXiv:1803.01297.

[29] Z. X. Zhao, Weak decays of heavy baryons in the light-front approach, Chin. Phys. C 42, 093101 (2018).

[30] T. Gutsche, M. A. Ivanov, J. G. Krner, and V. E. Lyubovitskij, Nonleptonic two-body decays of single heavy baryons $\Lambda_{Q}, \Xi_{Q}$, and $\Omega_{Q}(Q=b, c)$ induced by $W$ emission in the covariant confined quark model, Phys. Rev. D 98, 074011 (2018).

[31] H. Y. Cheng and C. K. Chua, Strong decays of charmed baryons in heavy hadron chiral perturbation theory, Phys. Rev. D 75, 014006 (2007).

[32] H. Y. Cheng and C. K. Chua, Strong decays of charmed baryons in heavy hadron chiral perturbation theory: An update, Phys. Rev. D 92, 074014 (2015).

[33] R. Aaij et al. (LHCb Collaboration), Observation of a New $\Xi_{b}^{-}$Resonance, Phys. Rev. Lett. 121, 072002 (2018).

[34] H. Y. Cheng, C. K. Chua, and C. W. Hwang, Covariant light front approach for $\mathrm{s}$ wave and $p$ wave mesons: Its application to decay constants and form-factors, Phys. Rev. D 69, 074025 (2004).

[35] H. Y. Cheng, C. Y. Cheung, and C. W. Hwang, Mesonic form-factors and the Isgur-Wise function on the light front, Phys. Rev. D 55, 1559 (1997).

[36] W. Jaus, Semileptonic decays of $B$ and $D$ mesons in the light front formalism, Phys. Rev. D 41, 3394 (1990).

[37] W. R. B. de Araujo, M. Beyer, T. Frederico, and H. J. Weber, Feynman versus Bakamjian-Thomas in light front dynamics, J. Phys. G 25, 1589 (1999).

[38] S. J. Brodsky, H. C. Pauli, and S. S. Pinsky, Quantum chromodynamics and other field theories on the light cone, Phys. Rep. 301, 299 (1998).

[39] W. Wang, F. S. Yu, and Z. X. Zhao, Weak decays of doubly heavy baryons: The $1 / 2 \rightarrow 1 / 2$ case, Eur. Phys. J. C 77, 781 (2017).

[40] W. Jaus, Relativistic constituent quark model of electroweak properties of light mesons, Phys. Rev. D 44, 2851 (1991).

[41] F. Schlumpf, Relativistic constituent quark model of electroweak properties of baryons, Phys. Rev. D 47, 4114 (1993); Erratum, Phys. Rev. D 49, 6246(E) (1994).

[42] D. Ebert, R. N. Faustov, and V. O. Galkin, Masses of tetraquarks with open charm and bottom, Phys. Lett. B 696, 241 (2011).

[43] W. Jaus, Semileptonic, radiative, and pionic decays of $B, B^{*}$ and $D, D^{*}$ mesons, Phys. Rev. D 53, 1349 (1996); Erratum, Phys. Rev. D 54, 5904(E) (1996).

[44] H. Y. Cheng and C. K. Chua, Covariant light front approach for $B \rightarrow K^{*} \gamma, K_{1} \gamma, K_{2}^{*} \gamma$ decays, Phys. Rev. D 69, 094007 (2004); Erratum, Phys. Rev. D 81, 059901 (2010). 
[45] M. Beneke, G. Buchalla, M. Neubert, and C. T. Sachrajda, QCD factorization in $B \rightarrow \pi K, \pi \pi$ decays and extraction of Wolfenstein parameters, Nucl. Phys. B606, 245 (2001).

[46] H. Y. Cheng, X. W. Kang, and F. Xu, Singly Cabibbosuppressed hadronic decays of $\Lambda_{c}^{+}$, Phys. Rev. D 97, 074028 (2018).

[47] J. Charles, A. Höcker, H. Lacker, S. Laplace, F. R. Le Diberder, J. Malclés, J. Ocariz, M. Pivk, and L. Roos
(CKMfitter Group), $C P$ violation and the CKM matrix: Assessing the impact of the asymmetric $B$ factories, Eur. Phys. J. C 41, 1 (2005), updated results available at http:// ckmfitter.in2p3.fr.

[48] M. Beneke, G. Buchalla, M. Neubert, and C. T. Sachrajda, QCD factorization for exclusive, nonleptonic $B$ meson decays: General arguments and the case of heavy light final states, Nucl. Phys. B591, 313 (2000). 